\title{
Holomorphic Methods in Analysis and Mathematical Physics
}

\author{
Brian C. Hall \\ University of Notre Dame \\ Department of Mathematics \\ Computing Center and Mathematics Building \\ Notre Dame, IN 46556, U.S.A. \\ bhall@nd.edu
}

December, 1999

\section{Contents}

\begin{tabular}{lll}
\hline 1 & Introduction & 2
\end{tabular}

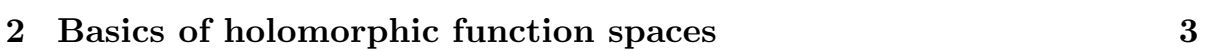

2.1 Exercises ..................... 8

3 Examples of holomorphic function spaces 9

3.1 The weighted Bergman spaces . . . . . . . . . . . . . . . . . . 9

3.2 The Segal-Bargmann spaces . . . . . . . . . . . . . . . . . . . . . 11

3.3 The Hardy space . . . . . . . . . . . . . . . . . . . . . . . . 13

3.4 Exercises . . . . . . . . . . . . . . . . . . . . 14

4 A special property of the Segal-Bargmann and weighted Bergman spaces 14

4.1 Unitarized translations on the Segal-Bargmann space . . . . . . . 15

4.2 Unitarized transformations of the weighted Bergman spaces . . . 17

4.3 Holomorphic equivalence . . . . . . . . . . . . . . . 18

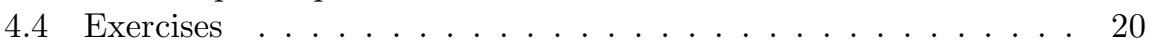

5 Canonical commutation relations 20

5.1 The standard form of the canonical commutation relations . . . . 20

5.2 The exponentiated form of the canonical commutation relations . 23

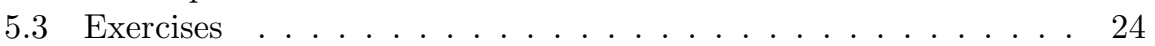


6 The Segal-Bargmann transform 26

6.1 Bargmann's extension of Fock's observation . . . . . . . . . . 26

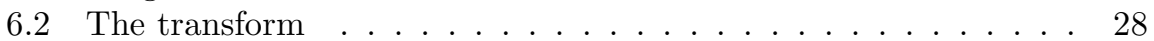

6.3 The "invariant" form of the Segal-Bargmann transform . . . . . 32

6.4 Historical remarks $\ldots \ldots \ldots \ldots \ldots$

6.5 Exercises $\ldots \ldots \ldots \ldots \ldots \ldots$

$\begin{array}{lll}7 & \text { Quantum mechanics and quantization } & 36\end{array}$

7.1 A brief survey of classical mechanics . . . . . . . . . . . . . 36

$7.2 \quad$ A very brief survey of quantum mechanic . . . . . . . . . . . . . 39

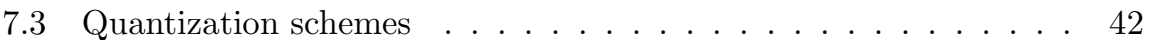

7.4 The significance of the Segal-Bargmann representation . . . . . . 45

7.5 Exercises $\ldots \ldots \ldots \ldots \ldots \ldots \ldots$

8 Toeplitz operators, anti-Wick ordering, and phase space probability densities 46

8.1 General theory of Toeplitz operators . . . . . . . . . . . . . 47

8.2 Toeplitz operators on the Segal-Bargmann space . . . . . . . . . 49

8.3 Wigner function and Husimi function . . . . . . . . . . . . . . 50

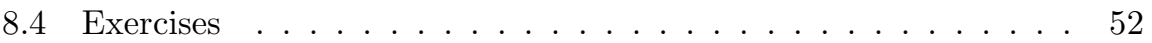

9 The Segal-Bargmann transform for compact Lie groups 53

9.1 Beyond the Canonical Commutation Relations . . . . . . . . 53

9.2 The transform for compact Lie groups $\ldots \ldots \ldots \ldots \ldots$

9.3 What is "right" about this transform? . . . . . . . . . . 58

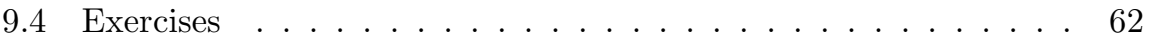

10 To infinity and beyond 63

10.1 The infinite-dimensional theory $\ldots \ldots \ldots$. . . . . . . 63

10.2 Coherent states $\ldots \ldots \ldots \ldots \ldots \ldots$

10.3 Kähler quantization . . . . . . . . . . . . . . . . . 68

\section{Introduction}

These notes are based on lectures that I gave at the Summer School in Mathematical Analysis at the Instituto de Matemáticas de la Universidad Nacional Autónoma de México, Unidad Cuernavaca, from June 8 to 18, 1998. I am grateful to Salvador Pérez Esteva and Carlos Villegas Blas for organizing the School and for inviting me, and to all the audience members for their attention and interest. I thank Steve Sontz for corrections to the manuscript.

The notes explain certain parts of the theory of holomorphic function spaces and the relation of that theory to quantum mechanics. The level is intended for beginning graduate students. I assume knowledge of the basics of holomorphic functions of one complex variable, Hilbert spaces, and measure theory. I do not assume any prior knowledge of holomorphic function spaces or quantum 
mechanics. I adopt throughout the physicists' convention that inner products be linear in the second factor and conjugate-linear in the first factor.

The notes include a number of exercises. Attempting the exercises will greatly increase the reader's understanding of the material-the best way to learn is to do. Exercises marked with a star are harder or assume knowledge of more advanced topics.

Much of the material in these notes has been known for some time, but has not previously, to my knowledge, been gathered in one place. My aim is to provide some of the conceptual and mathematical background needed to enter into the current research in this area. The last two sections give an introduction to more recent material.

\section{Basics of holomorphic function spaces}

This section is slightly more technical than most of the later ones, but unfortunately we need some preliminary results in order to get started.

Let $U$ be a non-empty open set in $\mathbb{C}^{d}$. Let $\mathcal{H}(U)$ denote the space of holomorphic (or complex analytic) functions on $U$. Recall that a function of several complex variables, $f: U \rightarrow \mathbb{C}$, is said to be holomorphic if $f$ is continuous and holomorphic in each variable with the other variables fixed. Let $\alpha$ be a continuous, strictly-positive function on $U$.

Definition 1 Let $\mathcal{H} L^{2}(U, \alpha)$ denote the space of $L^{2}$ holomorphic functions with respect to the weight $\alpha$, that is,

$$
\mathcal{H} L^{2}(U, \alpha)=\left\{\left.F \in \mathcal{H}(U)\left|\int_{U}\right| F(z)\right|^{2} \alpha(z) d z<\infty\right\} .
$$

Here and in what follows $d z$ denotes not a line integral, but rather the $2 d$ dimensional Lebesgue measure on $\mathbb{C}^{d}=\mathbb{R}^{2 d}$.

Theorem 2 1. For all $z \in U$, there exists a constant $c_{z}$ such that

$$
|F(z)|^{2} \leq c_{z}\|F\|_{L^{2}(U, \alpha)}^{2}
$$

for all $F \in \mathcal{H} L^{2}(U, \alpha)$.

2. $\mathcal{H} L^{2}(U, \alpha)$ is a closed subspace of $L^{2}(U, \alpha)$, and therefore a Hilbert space.

Point 1 says that pointwise evaluation is continuous. That is, for each $z \in U$, the map that takes a function $F \in \mathcal{H} L^{2}(U, \alpha)$ to the number $F(z)$ is a continuous linear functional on $\mathcal{H} L^{2}(U, \alpha)$. This is a crucial property of holomorphic function spaces, which is certainly false for ordinary (non-holomorphic) $L^{2}$ spaces.

Proof. (1) Let $P_{s}(z)$ be the "polydisk" of radius $s$, centered at $z$, that is,

$$
P_{s}(z)=\left\{v \in \mathbb{C}^{d}|| v_{k}-z_{k} \mid<s, \quad k=1, \cdots, d\right\} .
$$


Here $z=\left(z_{1}, \cdots, z_{d}\right)$, with each $z_{k} \in \mathbb{C}$. If $z \in U$, choose $s$ small enough so that $\overline{P_{s}(z)} \subset U$. I then claim that

$$
F(z)=\left(\pi s^{2}\right)^{-d} \int_{P_{s}(z)} F(v) d v .
$$

To verify this, consider at first the case $d=1$. Then we may expand $F$ in a Taylor series at $v=z$

$$
F(v)=F(z)+\sum_{n=1}^{\infty} a_{n}(v-z)^{n} .
$$

This series converges uniformly to $F$ on the compact set $\overline{P_{s}(z)} \subset U$. Thus when evaluating the integral on the RHS of (1D) we may interchange the integral with the sum. But now if we use polar coordinates with the origin at $z$, then $(v-z)^{n}=r^{n} e^{i n \theta}$. So for $n \geq 1$, the integral over $P_{s}(z)$ (which is just a disk of radius $s$ since $d=1$ ) give zero. So the only surviving term is the constant term $F(z)$, which gives $\pi s^{2}\left(\pi s^{2}\right)^{-1} F(z)$.

For the case $d>1$, we simply do the integral one variable at a time. By the $d=1$ case, when we do, say, the $v_{1}$ integral, this has the effect of setting $v_{1}=z_{1}$. So by the time we have done all $d$ integrals, we get just $F(z)$.

So now rewrite (11) in the form

$$
\begin{aligned}
F(z) & =\left(\pi s^{2}\right)^{-d} \int_{U} 1_{P_{s}(z)}(v) \frac{1}{\alpha(v)} F(v) \alpha(v) d v \\
& =\left(\pi s^{2}\right)^{-d}\left\langle 1_{P_{s}(z)} \frac{1}{\alpha}, F\right\rangle_{L^{2}(U, \alpha)},
\end{aligned}
$$

where $1_{P_{s}(z)}$ is the indicator function of $P_{s}(z)$, that is, the function which is one on $P_{s}(z)$ and zero elsewhere. Thus by the Schwarz inequality, we have

$$
|F(z)|^{2} \leq\left(\pi s^{2}\right)^{-2 d}\left\|1_{P_{s}(z)} \frac{1}{\alpha}\right\|_{L^{2}(U, \alpha)}^{2}\|F\|_{L^{2}(U, \alpha)}^{2} .
$$

Note that because $\overline{P_{s}(z)} \subset U$ and $\alpha$ is positive and continuous, $1 / \alpha$ is bounded on $P_{s}(z)$; thus the first $L^{2}$ norm is finite. Note also that we can take any $s$ we like here, provided only that $\overline{P_{s}(z)} \subset U$.

(2) Looking at the proof of (1), we see that in fact given $z \in U$, we can find a neighborhood $V$ of $z$ and a constant $d_{z}$ such that

$$
|F(v)|^{2} \leq d_{z}\|F\|_{L^{2}(U, \alpha)}^{2}
$$

for all $v \in V$ and all $F \in \mathcal{H} L^{2}(U, \alpha)$. (That is, the constant in (1) can be taken to be bounded in a neighborhood of each point.) So now suppose we have a sequence $F_{n} \in \mathcal{H} L^{2}(U, \alpha)$, and $F \in L^{2}(U, \alpha)$ such that $F_{n} \rightarrow F$ in $L^{2}(U, \alpha)$. Then $F_{n}$ is certainly a Cauchy sequence in $L^{2}$. But then

$$
\sup _{v \in V}\left|F_{n}(v)-F_{m}(v)\right| \leq \sqrt{d_{z}}\left\|F_{n}-F_{m}\right\|_{L^{2}(U, \alpha)} \rightarrow 0 \quad \text { as } n, m \rightarrow \infty .
$$


This shows that the sequence $F_{m}$ converges locally uniformly to some limit function, which must be $F$. (If $L^{2}$ limit and pointwise limit both exist they must be equal a.e.) But a standard theorem shows that a locally uniform limit of holomorphic functions is always holomorphic. (Use Morera's Theorem to show that the limit is still holomorphic in each variable.) So the limit function $F$ is actually in $\mathcal{H} L^{2}(U, \alpha)$, which shows that $\mathcal{H} L^{2}(U, \alpha)$ is closed.

Theorem 3 (Reproducing Kernel) Let $\mathcal{H} L^{2}(U, \alpha)$ be as above. Then there exists a function $K(z, w), z, w \in U$, with the following properties:

1. $K(z, w)$ is holomorphic in $z$ and anti-holomorphic in $w$, and satisfies

$$
K(w, z)=\overline{K(z, w)} .
$$

2. For each fixed $z \in U, K(z, w)$ is square-integrable $d \alpha(w)$. For all $F \in$ $\mathcal{H} L^{2}(U, \alpha)$

$$
F(z)=\int_{U} K(z, w) F(w) \alpha(w) d w
$$

3. If $F \in L^{2}(U, \alpha)$, let $P F$ denote the orthogonal projection of $F$ onto the closed subspace $\mathcal{H} L^{2}(U, \alpha)$. Then

$$
P F(z)=\int_{U} K(z, w) F(w) \alpha(w) d w .
$$

4. For all $z, u \in U$,

$$
\int_{U} K(z, w) K(w, u) \alpha(w) d w=K(z, u) .
$$

5. For all $z \in U$,

$$
|F(z)|^{2} \leq K(z, z)\|F\|^{2},
$$

and the constant $K(z, z)$ is optimal in the sense that for each $z \in U$ there exists a non-zero $F_{z} \in \mathcal{H} L^{2}(U, \alpha)$ for which equality holds.

6. Given any $z \in U$, if $\phi_{z}(\cdot) \in \mathcal{H} L^{2}(U, \alpha)$ satisfies

$$
F(z)=\int_{U} \overline{\phi_{z}(w)} F(w) \alpha(w) d w
$$

for all $F \in \mathcal{H} L^{2}(U, \alpha)$, then $\overline{\phi_{z}(w)}=K(z, w)$. 
Proof. We have already shown that evaluation at a point $z \in U$ is a continuous linear functional on $\mathcal{H} L^{2}(U, \alpha)$. Thus by the Riesz Theorem, this linear functional can be represented uniquely as inner product with some $\phi_{z} \in$ $\mathcal{H} L^{2}(U, \alpha)$, that is,

$$
\begin{aligned}
F(z) & =\left\langle\phi_{z}, F\right\rangle_{L^{2}(U, \alpha)} \\
& =\int_{U} \overline{\phi_{z}(w)} F(w) \alpha(w) d w .
\end{aligned}
$$

So we set $K(z, w)=\overline{\phi_{z}(w)}$. (I adopt the convention that the inner product be linear on the right and conjugate-linear on the left.) By its very construction, $K(z, w)$ satisfies Point 2 of the theorem and is anti-holomorphic in $w$.

Now we apply (2) to $\phi_{z}$ itself. Thus

$$
\begin{aligned}
\phi_{z}(w) & =\left\langle\phi_{w}, \phi_{z}\right\rangle_{L^{2}(U, \alpha)}={\overline{\left\langle\phi_{z}, \phi_{w}\right\rangle_{L^{2}(U, \alpha)}}}=\bar{\phi} w(z)
\end{aligned}
$$

Thus $\overline{K(z, w)}=K(w, z)$, and we have Point 1 .

For Point 3 , we consider two cases. If $F \in \mathcal{H} L^{2}(U, \alpha)$, then Point 3 says the same thing as Point 2. On the other hand, if $F \in\left[\mathcal{H} L^{2}(U, \alpha)\right]^{\perp}$, then the RHS of Point 3 is just $\left\langle\phi_{z}, F\right\rangle$ which is zero since $\phi_{z} \in \mathcal{H} L^{2}(U, \alpha)$. So the RHS of Point 3 is the identity on $\mathcal{H} L^{2}(U, \alpha)$ and zero on the orthogonal complement of $\mathcal{H} L^{2}(U, \alpha)$, so it must coincide with $P$.

Point 4 is just Point 2 applied to the square-integrable holomorphic function $K(w, u)$, viewing $w$ as the variable and $u$ as a parameter.

For Point 5 we note evaluation at $z$ is just inner product with an element $\phi_{z}$ of our Hilbert space. So the norm of this linear functional is just the norm of $\phi_{z}$. But

$$
\left\|\phi_{z}\right\|^{2}=\left\langle\phi_{z}, \phi_{z}\right\rangle_{L^{2}(U, \alpha)}=\phi_{z}(z)=K(z, z) .
$$

Saying that we have computed the norm of this linear functional means precisely that we have found the optimal constant in the inequality $|F(z)| \leq \sqrt{c_{z}}\|F\|$. The function $\phi_{z}$ itself is the one that gives equality in Point 5 .

For Point 6, note that if $\phi_{z}(\cdot)$ is in $\mathcal{H} L^{2}(U, \alpha)$ and satisfies $F(z)=\left\langle\phi_{z}, F\right\rangle$ for all $F \in \mathcal{H} L^{2}(U, \alpha)$, then $\left\langle\phi_{z}, F\right\rangle=\langle\overline{K(z, \cdot)}, F\rangle$ and $\left\langle\overline{K(z, \cdot)}-\phi_{z}, F\right\rangle=$ 0 , for all $F \in \mathcal{H} L^{2}(U, \alpha)$. Since $\overline{K(z, \cdot)}$ and $\phi_{z}$ are both in $\mathcal{H} L^{2}(U, \alpha)$, we may take $F=K(z, \cdot)-\phi_{z}(\cdot)$, which shows that $K(z, \cdot)-\phi_{z}(\cdot)=0$; that is, $\phi_{z}(w)=\overline{K(z, w)}$.

This theorem is really just the continuity of pointwise evaluation, together with the Riesz Theorem. The reproducing kernel is a useful way of encoding information about a holomorphic function space. Our next result gives us a way of calculating the reproducing kernel. 
Theorem 4 Let $\left\{e_{j}\right\}$ be any $O N$ basis for $\mathcal{H} L^{2}(U, \alpha)$. Then for all $z, w \in U$

$$
\sum_{j}\left|e_{j}(z) \overline{e_{j}(w)}\right|<\infty
$$

and

$$
K(z, w)=\sum_{j} e_{j}(z) \overline{e_{j}(w)}
$$

Proof. The annoying part of the proof is the convergence issues. Once this is done, verifying the formula for $K$ is fairly easy. So on a first reading you should skip to the last paragraph of the proof.

For any $f \in \mathcal{H} L^{2}(U, \alpha)$, Parseval's Theorem says that

$$
\sum_{j}\left|\left\langle f, e_{j}\right\rangle\right|^{2}=\|f\|^{2}
$$

Then for any $f, g \in \mathcal{H} L^{2}(U, \alpha)$, consider the Schwarz inequality in the space $l^{2}$ of square-summable sequences, applied to the sequences $\left|\left\langle f, e_{j}\right\rangle\right|$ and $\left|\left\langle g, e_{j}\right\rangle\right|$. This gives

$$
\sum_{j}\left|\left\langle f, e_{j}\right\rangle\left\langle e_{j}, g\right\rangle\right| \leq\|f\|\|g\|
$$

Taking $f=\phi_{z}$ and $g=\phi_{w}$ we get

$$
\sum_{j}\left|e_{j}(z) \overline{e_{j}(w)}\right| \leq\left\|\phi_{z}\right\|\left\|\phi_{w}\right\|<\infty
$$

So the sum is absolutely convergent for each $z$ and $w$.

Now think of the partial sums of $\sum_{j} e_{j}(z) \overline{e_{j}(w)}$ as functions of $w$ with $z$ fixed. Then the series is orthogonal and

$$
\begin{aligned}
\sum_{j}\left\|e_{j}(z) \overline{e_{j}(w)}\right\|_{L^{2}(w)}^{2} & =\sum_{j}\left\|\left\langle\phi_{z}, e_{j}\right\rangle e_{j}\right\|^{2} \\
& =\sum_{j}\left|\left\langle\phi_{z}, e_{j}\right\rangle\right|^{2}=\left\|\phi_{z}\right\|^{2}<\infty .
\end{aligned}
$$

So the series is actually $L^{2}$ convergent as a function of $w$ for fixed $z$. This shows (by the obvious analog of Theorem 2 for anti-holomorphic functions) that the sum is anti-holomorphic as a function of $w$ for each fixed $z$. Arguing in a similar way with the roles of $z$ and $w$ reversed shows that the sum is holomorphic as a function of $z$ for each fixed $w$.

So now that the unpleasant convergence issues are settled, let's prove the formula for $K$. In essence Theorem 1 says that any $F \in \mathcal{H} L^{2}(U, \alpha)$ is the sum of its projections onto the orthonormal basis elements $e_{j}$. If you prefer, you can 
verify the theorem first just for $F(z)=e_{k}(z)$ and then extend by linearity to arbitrary $F$.

For any $F \in \mathcal{H} L^{2}(U, \alpha)$ we have

$$
\begin{aligned}
F(z) & =\left\langle\phi_{z}, F\right\rangle=\sum_{j}\left\langle\phi_{z}, e_{j}\right\rangle\left\langle e_{j}, F\right\rangle \\
& =\sum_{j} e_{j}(z) \int_{U} \overline{e_{j}(w)} F(w) \alpha(w) d w \\
& =\int_{U}\left[\sum_{j} e_{j}(z) \overline{e_{j}(w)}\right] F(w) \alpha(w) d w .
\end{aligned}
$$

In the first line we have used the basic property of the $\phi_{z}$ 's and Parseval's Theorem. In the second line we have used the basic property of $\phi_{z}$ to evaluate $\left\langle\phi_{z}, e_{j}\right\rangle$, and we have written out $\left\langle e_{j}, F\right\rangle$ as an integral. In the third line we have interchanged the sum and integral, as justified by the $L^{2}$ convergence of the sum. Finally, then, by Point 6 of the last theorem we conclude that the quantity in square brackets must be $K(z, w)$.

Remark. Most of this time, this formula for the reproducing kernel is not especially useful, since (1) you can't usually find explicitly an orthonormal basis, and (2) even if you could, you probably couldn't compute the sum. But it will give explicit formulas for the reproducing kernel in certain important cases.

\section{$2.1 \quad$ Exercises}

Exercise 1 Show that for all $z \in U$, there exist constants $c_{z, k}$ such that

$$
\left|\frac{\partial F}{\partial z_{k}}(z)\right|^{2} \leq c_{z, k}\|F\|^{2} .
$$

(You may do this just in the case $d=1$ if it makes things easier.)

Exercise 2 Show that $\mathcal{H} L^{2}(\mathbb{C}, 1)=\{0\}$.

Hint: Suppose $F \in \mathcal{H} L^{2}(\mathbb{C}, 1)$. Use Theorem 目 to show that $F$ must be bounded.

Exercise $3{ }^{*}$ Consider the measure $\mu$ on the plane with the property that for all bounded measurable functions $f$,

$$
\int_{\mathbb{C}} f d \mu=\int_{\mathbb{R}} f(x, 0) d x .
$$

(So this measure is concentrated on the real axis.) Show that $\mathcal{H}(\mathbb{C}) \cap L^{2}(\mathbb{C}, \mu)$ is dense in $L^{2}(\mathbb{C}, \mu)$. In this case $\mathcal{H}(\mathbb{C}) \cap L^{2}(\mathbb{C}, \mu)$ is not a Hilbert space and pointwise evaluation is not continuous. (This is why we consider only measures that have a positive density with respect to Lebesgue measure on $\mathbb{C}^{d}$.) 


\section{Examples of holomorphic function spaces}

\subsection{The weighted Bergman spaces}

Definition 5 The weighted Bergman spaces are the spaces

$$
\mathcal{H} L^{2}\left(\mathbb{D},\left(1-|z|^{2}\right)^{a}\right), \quad a>-1
$$

where $\mathbb{D}$ is the unit disk,

$$
\mathbb{D}=\{z \in \mathbb{C}|| z \mid<1\}
$$

Here the restriction $a>-1$ is needed to get a non-zero space. The weighted Bergman spaces are important in operator theory and in representation theory. We will compute the reproducing kernel for the weighted Bergman spaces just in the case $a=0$, in which case the space is the standard Bergman space. We will denote this space $\mathcal{H} L^{2}(\mathbb{D})$, with the weight 1 being understood.

Step 1. Show that $\left\{z^{n}\right\}_{n=0}^{\infty}$ is an orthogonal basis for $\mathcal{H} L^{2}(\mathbb{D})$.

We first check orthogonality, computing the integral in polar coordinates.

$$
\begin{aligned}
\left\langle z^{n}, z^{m}\right\rangle & =\int_{0}^{2 \pi} \int_{0}^{1} r^{n} e^{-i n \theta} r^{m} e^{i m \theta} r d r d \theta \\
& =\int_{0}^{1} r^{n+m+1} \int_{0}^{2 \pi} e^{i(m-n) \theta} d \theta d r \\
& =0 \quad(n \neq m) .
\end{aligned}
$$

We now need to show that the $z^{n}$ 's span a dense subspace of $\mathcal{H} L^{2}(\mathbb{D})$. It suffices to show that if $F \in \mathcal{H} L^{2}(\mathbb{D})$ and $\left\langle z^{n}, F\right\rangle=0$ for all $n$, then $F=0$. So suppose $F \in \mathcal{H} L^{2}(\mathbb{D})$. We expand $F$ in a power series

$$
F(z)=\sum_{n=0}^{\infty} c_{n} z^{n} .
$$

This series converges uniformly on compact subsets of $\mathbb{D}$. Now compute

$$
\begin{aligned}
\left\langle z^{m}, F\right\rangle & =\int_{0}^{1} \int_{0}^{2 \pi} r^{m} e^{-i m \theta} F\left(r e^{i \theta}\right) r d r d \theta \\
& =\lim _{a \rightarrow 1} \int_{0}^{a} \int_{0}^{2 \pi} r^{m} e^{-i m \theta} F\left(r e^{i \theta}\right) r d r d \theta
\end{aligned}
$$

where the last equality is by Dominated Convergence. But now the series (4) converges uniformly on the set $r \leq a$, and so we may interchange integral and sum to get

$$
\begin{aligned}
\left\langle z^{m}, F\right\rangle & =\lim _{a \rightarrow 1} \sum_{n=0}^{\infty} \int_{0}^{a} \int_{0}^{2 \pi} r^{m} e^{-i m \theta} c_{n} r^{n} e^{i n \theta} r d r d \theta \\
& =\lim _{a \rightarrow 1} \sum_{n=0}^{\infty} c_{n} \int_{0}^{a} r^{n+m+1} \int_{0}^{2 \pi} e^{i(n-m) \theta} d \theta d r
\end{aligned}
$$


But the $\theta$ integral gives zero except when $n=m$. So only one term in the sum survives, and we can then let $a$ tend to 1 to get

$$
\begin{aligned}
\left\langle z^{m}, F\right\rangle & =2 \pi c_{m} \int_{0}^{1} r^{2 m+1} d r \\
& =2 \pi c_{m} \frac{1}{2 m+2}=\frac{\pi c_{m}}{m+1} .
\end{aligned}
$$

So if $\left\langle z^{m}, F\right\rangle=0$ for all $m$, then $c_{m}=0$ for all $m$, in which case $F$ is identically zero. So $\left\{z^{m}\right\}$ is a basis.

Step 2. Normalize.

Compute that

$$
\begin{aligned}
\left\|z^{n}\right\|^{2} & =\int_{0}^{1} \int_{0}^{2 \pi} r^{2 n} r d r d \theta \\
& =2 \pi \frac{1}{2 n+2}=\frac{\pi}{n+1} .
\end{aligned}
$$

So

$$
\left\{z^{n} \sqrt{\frac{n+1}{\pi}}\right\}_{n=0}^{\infty}
$$

is an orthonormal basis for $\mathcal{H} L^{2}(\mathbb{D})$.

Step 3. Compute reproducing kernel.

According to our theorem, we may now compute the reproducing kernel as

$$
\begin{aligned}
K(z, w) & =\sum_{n=0}^{\infty} z^{n} \sqrt{\frac{n+1}{\pi}} \bar{w}^{n} \sqrt{\frac{n+1}{\pi}} \\
& =\frac{1}{\pi} \sum_{n=0}^{\infty}(n+1)(z \bar{w})^{n} .
\end{aligned}
$$

So now let us consider the function

$$
\begin{aligned}
f(\xi) & =\sum_{n=0}^{\infty}(n+1) \xi^{n} \\
& =\sum_{n=0}^{\infty} \frac{d}{d \xi} \xi^{n+1} \\
& =\frac{d}{d \xi} \sum_{n=0}^{\infty} \xi^{n+1}=\frac{d}{d \xi}\left(\xi+\xi^{2}+\xi^{3}+\cdots\right) .
\end{aligned}
$$

Adding a 1 inside the derivative (harmless since the derivative of 1 is zero) we get

$$
f(\xi)=\frac{d}{d \xi} \frac{1}{1-\xi}=\frac{1}{(1-\xi)^{2}} .
$$


Conclusion 6 The reproducing kernel for the standard Bergman space is

$$
K(z, w)=\frac{1}{\pi} \frac{1}{(1-z \bar{w})^{2}} .
$$

Thus in particular,

$$
|F(z)|^{2} \leq \frac{1}{\pi\left(1-|z|^{2}\right)^{2}}\|F\|^{2},
$$

for all $F \in \mathcal{H} L^{2}(\mathbb{D})$ and all $z \in \mathbb{D}$.

\subsection{The Segal-Bargmann spaces}

Definition 7 The Segal-Bargmann spaces are the holomorphic function spaces

$$
\mathcal{H} L^{2}\left(\mathbb{C}^{d}, \mu_{t}\right),
$$

where

$$
\mu_{t}(z)=(\pi t)^{-d} e^{-|z|^{2} / t} .
$$

Here $|z|^{2}=\left|z_{1}\right|^{2}+\cdots+\left|z_{d}\right|^{2}$ and $t$ is a positive number.

We will now compute the reproducing kernel for the Segal-Bargmann space. We consider at first just the case $d=1$.

Step 1. Show that $\left\{z^{n}\right\}_{n=0}^{\infty}$ is a basis for the Segal-Bargmann space, with $d=1$.

The proof of this is nearly the same as the proof of Step 1 in the computation of the reproducing kernel for the standard Bergman space, and is omitted.

Step 2. Normalize. $d=1)$

We compute $\left\|z^{n}\right\|^{2}$ by induction on $n$. For $n=0$ we observe that (with

$$
\begin{aligned}
\int_{\mathbb{C}}(1)^{2} \mu_{t}(z) d z & =\frac{1}{\pi t} \int_{0}^{2 \pi} \int_{0}^{\infty} e^{-r^{2} / t} r d r d \theta \\
& =\left.\frac{2 \pi}{\pi t}\left(-\frac{t}{2}\right) \lim _{A \rightarrow \infty} e^{-r^{2} / t}\right|_{0} ^{A} \\
& =-\lim _{A \rightarrow \infty}\left[e^{-A^{2} / 2}-1\right]=1
\end{aligned}
$$

Next we compute that for $n>0$,

$$
\begin{aligned}
\left\|z^{n}\right\|^{2} & =\int_{\mathbb{C}}\left|z^{n}\right|^{2} \mu_{t}(z) d z=\frac{1}{\pi t} \int_{0}^{2 \pi} \int_{0}^{\infty} e^{-r^{2} / t} r^{2 n+1} d r d \theta \\
& =\frac{2}{t} \int_{0}^{\infty} r^{2 n}\left(e^{-r^{2} / t} r\right) d r
\end{aligned}
$$


Integrating by parts gives

$$
\begin{aligned}
\left\|z^{n}\right\|^{2} & =-\frac{2}{t} \int_{0}^{\infty}\left(2 n r^{2 n-1}\right)\left(-\frac{t}{2} e^{-r^{2} / t}\right) d r \\
& =\frac{2}{t}(n t) \int_{0}^{\infty} e^{-r^{2} / t} r^{2(n-1)+1} d r \\
& =n t\left\|z^{n-1}\right\|^{2} .
\end{aligned}
$$

Thus we will have

$$
\left\|z^{n}\right\|^{2}=n ! t^{n}
$$

and so

$$
\left\{\frac{z^{n}}{\sqrt{n ! t^{n}}}\right\}_{n=0}^{\infty}
$$

is an orthonormal basis for $\mathcal{H} L^{2}\left(\mathbb{C}^{d}, \mu_{t}\right)$.

Step 3. Compute the reproducing kernel.

Our formula for the reproducing kernel is now

$$
\begin{aligned}
K(z, w) & =\sum_{n=0}^{\infty} \frac{z^{n}}{\sqrt{n ! t^{n}}} \frac{\overline{w^{n}}}{\sqrt{n ! t^{n}}} \\
& =\sum_{n=0}^{\infty} \frac{1}{n !}\left(\frac{z \bar{w}}{t}\right)^{n}=e^{z \bar{w} / t} .
\end{aligned}
$$

So we have computed the reproducing kernel explicitly for the case $d=1$. For general $d$ we have the following result.

Theorem 8 For all $d \geq 1$, the reproducing kernel for the space $\mathcal{H} L^{2}\left(\mathbb{C}^{d}, \mu_{t}\right)$ is given by

$$
K(z, w)=e^{z \cdot \bar{w} / t},
$$

where $z \cdot \bar{w}=z_{1} \bar{w}_{1}+\cdots+z_{d} \bar{w}_{d}$. In particular, we have the pointwise bounds

$$
|F(z)|^{2} \leq e^{|z|^{2} / t}\|F\|^{2}
$$

for all $F \in \mathcal{H} L^{2}\left(\mathbb{C}^{d}, \mu_{t}\right)$ and all $z \in \mathbb{C}^{d}$.

Note that the bounds are reasonable, since $|F(z)|^{2}$ is required to be squareintegrable against the density $e^{-|z|^{2} / t}$. We will derive the reproducing kernel of the Segal-Bargmann in two other ways, one in Section 1 and one in Section 6 .

Proof. We have already proved this for the case $d=1$. For general $d$ we note first of all that $\overline{K(z, w)}$, with $K$ as given in the theorem, is certainly holomorphic and square-integrable against $\mu_{t}$ as a function of $w$ for each fixed $z$. (The function $\overline{K(z, w)}$ grows only exponentially with $w$ for each fixed $z$, and 
so it is square-integrable against $e^{-|z|^{2} / t}$.) Note that the $d$-dimensional density $\mu_{t}(z)$ just factors as a product of the 1-dimensional densities in each variable. Thus

$\int_{\mathbb{C}^{d}} e^{z \cdot \bar{w} / t} F(w) \mu_{t}(w) d w=\int_{\mathbb{C}} \cdots \int_{\mathbb{C}} e^{z_{1} \bar{w}_{1} / t} \cdots e^{z_{d} \bar{w}_{d} / t} F\left(w_{1}, \cdots, w_{d}\right) \frac{d w_{1}}{\pi t} \cdots \frac{d w_{d}}{\pi t}$.

Now, $F\left(w_{1}, \cdots, w_{d}\right)$ is holomorphic in each variable with the others fixed. So provided that $F$ is square-integrable with respect to $(\pi t)^{-1} e^{-\left|w_{i}\right|^{2} / t} d w_{i}$ with respect to each $w_{i}$ with the other variables fixed, then we may apply the onedimensional result $d$ times, which gives simply

$$
\int_{\mathbb{C}^{d}} e^{z \cdot \bar{w} / t} F(w) \mu_{t}(w) d w=F\left(z_{1}, \cdots, z_{d}\right) .
$$

This, by Point 6 of Theorem 3, would show that $K(z, w)=e^{z \cdot \bar{w} / t}$, as claimed.

So let us assume at first that $F$ is a polynomial. Then $F$ is a polynomial in each variable with the others fixed, and so there is no trouble with square-integrability. But a Taylor series argument, similar to the density of polynomials in the standard Bergman space, shows that polynomials are dense in $\mathcal{H} L^{2}\left(\mathbb{C}^{d}, \mu_{t}\right)$. So since $e^{z \cdot \bar{w} / t}$ is $\mu_{t}$-square-integrable as a function of $w$, if (5) holds on a dense set, it must hold for all $F \in \mathcal{H} L^{2}\left(\mathbb{C}^{d}, \mu_{t}\right)$.

\subsection{The Hardy space}

This space is not quite an example of the sort considered in Section 2, but is an important space which I therefore wish to introduce. It is also interesting to contrast this example with Exercise 3 of Section 2 .

Definition 9 The Hardy space is the space of holomorphic functions $F$ on the unit disk $\mathbb{D}$ such that

$$
\sup _{r<1} \int_{0}^{2 \pi}\left|F\left(r e^{i \theta}\right)\right|^{2} d \theta<\infty .
$$

So this is almost like an $L^{2}$ space of holomorphic functions on $\mathbb{D}$ with respect to a measure $\mu$, except that the measure is not a measure on $\mathbb{D}$, but is a measure on the boundary of $\mathbb{D}$. It is not too hard, using Taylor series, to show that for any

$F \in \mathcal{H}(\mathbb{D}), \int_{0}^{2 \pi}\left|F\left(r e^{i \theta}\right)\right|^{2} d \theta$ is an increasing function of $r$. Thus the supremum in the definition is equal to the limit as $r$ approaches one. We then define a norm and an inner product on the Hardy space by defining

$$
\|F\|^{2}=\lim _{r \rightarrow 1} \int_{0}^{2 \pi}\left|F\left(r e^{i \theta}\right)\right|^{2} d \theta
$$

and

$$
\langle F, G\rangle=\lim _{r \rightarrow 1} \int_{0}^{2 \pi} \overline{F\left(r e^{i \theta}\right)} G\left(r e^{i \theta}\right) d \theta .
$$


Using Taylor series again, it is not too hard to show that the limit defining the inner product exists whenever $F$ and $G$ are in the Hardy space.

Even though it is not a holomorphic function space of the sort considered in Section 2, nevertheless the Hardy space has all the same properties of those spaces: pointwise evaluation is continuous, there is a reproducing kernel with all the properties of Theorem 3 (substituting the Hardy space itself for $\mathcal{H} L^{2}(U, \alpha)$ everywhere), the Hardy space is a Hilbert space. I will not give the proofs here, but they require no more than the Cauchy integral formula and Taylor series. See Exercise 5 .

\subsection{Exercises}

Exercise 4 Verify directly that the formula for the reproducing kernel of the standard Bergman space is correct when $z=0$. (Recall Point 6 of Theorem G.) Do the same for the Segal-Bargmann space with $d=1$.

Exercise 5 Show that for $F \in \mathcal{H}(\mathbb{D}), \int_{0}^{2 \pi}\left|F\left(r e^{i \theta}\right)\right|^{2} d \theta$ is increasing with $r$. Show that for all $z \in \mathbb{D}$, there is a constant $c_{z}$ such that

$$
|F(z)|^{2} \leq c_{z} \lim _{r \rightarrow 1} \int_{0}^{2 \pi}\left|F\left(r e^{i \theta}\right)\right|^{2} d \theta
$$

for all $F$ in the Hardy space.

Exercise 6 Compute the reproducing kernel for the Hardy space. You may assume that the standard formula for the reproducing kernel holds, even though the Hardy space is not a "standard" holomorphic function space.

Exercise 7 Compute the reproducing kernel for the weighted Bergman spaces.

\section{A special property of the Segal-Bargmann and weighted Bergman spaces}

One may well ask why we consider the examples we did. That is, why use the particular densities that appear in the weighted Bergman and Segal-Bargmann spaces? Why not some other densities? We will examine here one special property of the Segal-Bargmann spaces that holds (essentially) only for those

spaces. Something similar holds for the weighted Bergman spaces, which we will touch on briefly. 


\subsection{Unitarized translations on the Segal-Bargmann space}

Definition 10 Consider the Segal-Bargmann space $\mathcal{H} L^{2}\left(\mathbb{C}^{d}, \mu_{t}\right)$, for some $t>$ 0 . Now for each $a \in \mathbb{C}^{d}$, define a linear transformation $T_{a}: \mathcal{H} L^{2}\left(\mathbb{C}^{d}, \mu_{t}\right) \rightarrow$ $\mathcal{H} L^{2}\left(\mathbb{C}^{d}, \mu_{t}\right)$ by

$$
T_{a} F(z)=e^{-|a|^{2} / 2 t} e^{\bar{a} \cdot z / t} F(z-a) .
$$

At the moment it is not obvious that $T_{a}$ actually maps the Segal-Bargmann space into itself. But in fact $T_{a}$ is unitary for each $a$.

Theorem 11 1. For all $a \in \mathbb{C}^{d}, T_{a}$ is unitary on $\mathcal{H} L^{2}\left(\mathbb{C}^{d}, \mu_{t}\right)$.

2. For all $a, b \in \mathbb{C}^{d}$,

$$
T_{a} T_{b}=e^{i \operatorname{Im}(a \cdot \bar{b}) / t} T_{a+b}
$$

Let us discuss this theorem before proving it. To prove that $T_{a}$ is isometric, we need only check that

$$
\left|e^{-|a|^{2} / 2 t} e^{\bar{a} \cdot z / t}\right|^{2}=\frac{\mu_{t}(z-a)}{\mu_{t}(z)}
$$

which is an easy calculation. (See the proof.) So $T_{a}$ is a "unitarized translation"; that is, it first translates $F$ by $a$, and then multiplies by something which makes the transformation unitary. Note that translation itself is not unitary, since our measure $\mu_{t}$ is not translation-invariant. And we may not use the translationinvariant measure (Lebesgue measure) because then the only square-integrable holomorphic function would be 0. (Recall Exercise 2.)

If we were working with ordinary (non-holomorphic) $L^{2}$ spaces, then we could define unitarized translations for any strictly positive density $\alpha$, simply by taking as our "multiplier" (i.e., the thing that we multiply $F(z-a)$ by) to be $[\alpha(z-a) / \alpha(z)]^{1 / 2}$. But if we want to map the holomorphic subspace into itself, then the multiplier must be holomorphic. The special property of the Segal-Bargmann space is that there exists a holomorphic function $\phi_{a}$ such that $\left|\phi_{a}(z)\right|^{2}=\mu_{t}(z-a) / \mu_{t}(z)$. (If you take just any old positive function $\gamma(z)$, then there will usually not exist any holomorphic function $\phi$ with $|\phi(z)|^{2}=$ $\gamma(z)$. See Exercise 8.) This special property holds only for spaces which are holomorphically equivalent to one of the Segal-Bargmann spaces. See Section 4.3 .

Point 2 of the theorem says that the $T_{a}$ 's multiply the way ordinary translations do, modulo a constant. You might think that we could alter the definition of the $T_{a}$ 's by a constant to make them multiply exactly as ordinary translations, but this is impossible. After all, Point 2 implies that in general $T_{a}$ fails to commute with $T_{b}$, which means that a constant times $T_{a}$ will fail to commute with a constant times $T_{b}$, which means that we cannot have

$$
\left(\alpha T_{a}\right)\left(\beta T_{b}\right)=\gamma T_{a+b}=\gamma T_{b+a}=\left(\beta T_{b}\right)\left(\alpha T_{a}\right)
$$


in general. Point 2 says that the $T_{a}$ 's constitute a projective unitary representation of the additive group $\mathbb{C}^{d}$.

[A classic paper of Bargmann [B4] determines for which groups it is always possible to choose constants so that a projective unitary representation becomes an ordinary unitary representation. We see that $\mathbb{C}^{d}$ is not such a group. The rotation group $S O(3)$ is not such a group either, but $S U(2)$ is. This theory helps to explain the physical significance of "spin" in quantum physics.]

Proof of Theorem 11. (1) Recall as in the formula for the reproducing kernel that $\bar{a} \cdot z=\bar{a}_{1} z_{1}+\cdots+\bar{a}_{d} z_{d}$. This is a complex-valued quantity, whose real part is $\operatorname{Re} \bar{a} \cdot z=\sum_{k=1}^{d} \operatorname{Re} a_{k} \operatorname{Re} z_{k}+\operatorname{Im} a_{k} \operatorname{Im} z_{k}$. Thus

$$
\begin{aligned}
|z-a|^{2} & =\sum_{k=1}^{d} \overline{\left(z_{k}-a_{k}\right)}\left(z_{k}-a_{k}\right) \\
& =\sum_{k=1}^{d}\left(\bar{z}_{k} z_{k}+\bar{a}_{k} a_{k}-\bar{a}_{k} z_{k}-\bar{z}_{k} a_{k}\right) \\
& =|z|^{2}+|a|^{2}-2 \operatorname{Re} \bar{a} \cdot z
\end{aligned}
$$

Now by brute force calculation we find that

$$
\begin{aligned}
\left|e^{-|a|^{2} / 2 t} e^{\bar{a} \cdot z / t} F(z-a)\right|^{2} e^{-|z|^{2} / t} & =e^{-|a|^{2} / t} e^{2 \operatorname{Re}(\bar{a} \cdot z) / t} e^{-|z|^{2} / t}|F(z-a)|^{2} \\
& =e^{-|z-a|^{2} / t}|F(z-a)|^{2}
\end{aligned}
$$

Multiplying by $(\pi t)^{-d}$ and integrating shows that $\left\|T_{a} F\right\|^{2}=\|F\|^{2}$. Thus $T_{a}$ is an isometric map of $\mathcal{H} L^{2}\left(\mathbb{C}^{d}, \mu_{t}\right)$ to itself. The invertibility of $T_{a}$ will follow from Point 2, which implies that $\left(T_{a}\right)^{-1}$ is a multiple of $T_{-a}$.

(2) We compute that

$$
\begin{aligned}
T_{b} F(z) & =e^{-|b|^{2} / 2 t} e^{\bar{b} \cdot z / t} F(z-b) \\
T_{a} T_{b} F(z) & =e^{-|a|^{2} / 2 t} e^{\bar{a} \cdot z / t} e^{-|b|^{2} / 2 t} e^{\bar{b} \cdot(z-a) / t} F(z-a-b) \\
& =e^{-|a|^{2} / 2 t} e^{-|b|^{2} / 2 t} e^{-\bar{b} \cdot a / t} e^{(\bar{a}+\bar{b}) \cdot z / t} F(z-(a+b)) .
\end{aligned}
$$

But $|a+b|^{2}=|a|^{2}+|b|^{2}+2 \operatorname{Re} \bar{b} \cdot a$. Thus the first three factors in the expression for $T_{a} T_{b}$ will combine to give $\exp \left(-|a+b|^{2} / 2 t\right)$, with a leftover factor of $\exp (-i \operatorname{Im}(a \cdot \bar{b}) / t)$. So

$$
T_{a} T_{b} F(z)=e^{-i \operatorname{Im}(a \cdot \bar{b}) / t} T_{a+b} F(z),
$$

which is what we want to prove.

One can use these unitarized translations to give another derivation of the reproducing kernel of the Segal-Bargmann space, as follows. I do this for the case $d=1$; the general case can be reduced to this case as in Section 3. Using 
polar coordinates and Taylor series one may easily prove (Exercise 4 of Section 3) that

$$
F(0)=\int_{\mathbb{C}} 1 \cdot F(w) \mu_{t}(w) d w .
$$

But then

$$
T_{-a} F(0)=\int_{\mathbb{C}} T_{-a} F(w) \mu_{t}(w) d w
$$

or

$$
e^{-|a|^{2} / 2 t} F(a)=\int_{\mathbb{C}} e^{-|a|^{2} / 2 t} e^{-\bar{a} w / t} F(w+a) \mu_{t}(w) d w .
$$

Canceling the factors of $e^{-|a|^{2} / 2 t}$ gives

$$
F(a)=\int_{\mathbb{C}} e^{-\bar{a} w / t} F(w+a) \mu_{t}(w) d w .
$$

Letting $u=w+a$ and then multiplying and dividing by $\mu_{t}(u)$ gives

$$
\begin{aligned}
F(a) & =\int_{\mathbb{C}} e^{-\bar{a}(u-a) / t} F(u) \frac{\mu_{t}(u-a)}{\mu_{t}(u)} \mu_{t}(u) d u . \\
& =\int_{\mathbb{C}} e^{-\bar{a} u / t} e^{|a|^{2} / t} F(u) e^{-|a|^{2} / t} e^{(\bar{a} u+a \bar{u}) / t} \mu_{t}(u) d u \\
& =\int_{\mathbb{C}} e^{a \bar{u} / t} F(u) d u .
\end{aligned}
$$

where we have used the calculation: $\mu_{t}(u-a) / \mu_{t}(u)=e^{-|a|^{2} / t} e^{(\bar{a} u+a \bar{u}) / t}$. Since $\overline{e^{a \bar{u} / t}}$ is holomorphic and $\mu_{t}$-square-integrable as a function of $u$ for each $a$, Point 6 of Theorem 3 shows that $K(a, u)=e^{a \bar{u} / t}$.

The seemingly miraculous part of this calculation is that coefficient of $F(u)$ in this formula for $F(a)$ comes out to be anti-holomorphic as a function of $u$ for each $a$. But this is not coincidence. This method "automatically" will give an expression of the form $F(a)=\int \psi_{a}(u) F(u) \mu_{t}(u) d u$, which in turns gives the estimate $|F(a)|^{2} \leq\left\|\psi_{a}\right\|^{2}\|F\|^{2}$. Using the fact that $T_{a}$ is unitary and the fact that $K(0, w)=1$, it is possible to show that this estimate is sharp, which can happen only if $\psi_{a}(u)=K(a, u)$.

\subsection{Unitarized transformations of the weighted Bergman spaces}

There is an analogous theory for the weighted Bergman spaces. Recall the definition of fractional linear transformations. These are transformations of the form

$$
z \rightarrow \frac{a z+b}{c z+d}
$$


with $a d-b c=1$. (You could allow constants with $a d-b c \neq 1$, but you don't get any new transformations this way.) We wish to consider those fractional linear transformations that map the unit disk $\mathbb{D}$ onto itself. These are precisely the fractional linear transformations where $a, b, c, d$ form a matrix of the form

$$
\left\{\left.\left(\begin{array}{cc}
a & b \\
\bar{b} & \bar{a}
\end{array}\right)|| a\right|^{2}-|b|^{2}=1\right\}
$$

The set of matrices of this form make up a group denoted $S U(1,1)$. If $g$ is a matrix of the form (7) we will let $g \cdot z$ denote the result of the corresponding fractional linear transformation, namely, $(a z+b) /(\bar{b} z+\bar{a})$.

Theorem 12 Fix a number $a>-1$ and consider the weighted Bergman spaces

$$
\mathcal{H} L^{2}\left(\mathbb{D},\left(1-|z|^{2}\right)^{a}\right) .
$$

For each $g \in S U(1,1)$ there exists a holomorphic function $\phi_{g}$, unique up to a constant, such that the map

$$
U_{g} F(z)=\phi_{g}(z) F\left(g^{-1} \cdot z\right)
$$

is unitary on $\mathcal{H} L^{2}\left(\mathbb{D},\left(1-|z|^{2}\right)^{a}\right)$. For all $g, h \in S U(1,1)$ there exists a real number $\theta$ such that

$$
U_{g} U_{h}=e^{i \theta} U_{g h}
$$

Here $g^{-1}$ denotes a matrix inverse and $g h$ denotes a matrix product. I will not prove this theorem. A similar theory holds for weighted Bergman spaces on the unit ball in $\mathbb{C}^{d}$, with the group $S U(n, 1)$ replacing $S U(1,1)$.

[The operators $U_{g}$ constitute a projective unitary representation of $S U(1,1)$. For certain discrete values of $a$, this projective unitary representation can be made into an ordinary unitary representation. That is, one can choose constants so that $U_{g} U_{h}=U_{g h}$ for all $g$ and $h$. The resulting unitary representations of $S U(1,1)$ are called the holomorphic discrete series, first described by (who else?) Bargmann [B5].]

\subsection{Holomorphic equivalence}

Let us return briefly to the general setting $\mathcal{H} L^{2}(U, \alpha)$. Let $\phi$ be a nowhere zero holomorphic function on $U$. Then

$$
\int_{U}|F(z)|^{2} \alpha(z) d z=\int_{U}|\phi(z) F(z)|^{2} \frac{1}{|\phi(z)|^{2}} \alpha(z) d z .
$$

So the map $F \rightarrow \phi F$ is a unitary map of $\mathcal{H} L^{2}(U, \alpha)$ onto $\mathcal{H} L^{2}\left(U, \alpha /|\phi|^{2}\right)$, whose inverse is the map $G \rightarrow \frac{1}{\phi} G$. 
Definition 13 The holomorphic function spaces $\mathcal{H} L^{2}(U, \alpha)$ and $\mathcal{H} L^{2}(U, \beta)$ are said to be holomorphically equivalent if there exists a nowhere zero holomorphic function $\phi$ on $U$ such that

$$
\beta(z)=\frac{\alpha(z)}{|\phi(z)|^{2}}
$$

for all $z \in U$. The holomorphic equivalence between $\mathcal{H} L^{2}(U, \alpha)$ and $\mathcal{H} L^{2}(U, \beta)$ is the unitary map $F \rightarrow \phi F$.

Theorem 14 Suppose $\alpha$ is a strictly positive smooth function on $\mathbb{C}$ such that:

1. $\mathcal{H} L^{2}(\mathbb{C}, \alpha)$ contains at least one non-zero function, and

2. For all $a \in \mathbb{C}$ there exists a holomorphic function $\phi_{a}$ such that the map

$$
T_{a} F(z)=\phi_{a}(z) F(z-a)
$$

is unitary on $\mathcal{H} L^{2}(\mathbb{C}, \alpha)$.

Then $\mathcal{H} L^{2}(\mathbb{C}, \alpha)$ is holomorphically equivalent to one of the Segal-Bargmann spaces.

The proof of this theorem is left as an exercise. You should use Exercise 8. Then let $\beta(z)=\log \alpha(z)$. If the hypotheses of the theorem hold, show that $\Delta \beta(z)=c$ (a constant). Here $\Delta$ is the standard Laplacian operator, $\Delta=\partial^{2} / \partial x^{2}+\partial^{2} / \partial y^{2}$.

Theorem 15 Suppose $\alpha$ is a strictly positive smooth function on $\mathbb{D}$ such that

1. $\mathcal{H} L^{2}(\mathbb{D}, \alpha)$ contains at least one non-zero function, and

2. For all $g \in S U(1,1)$ there exists a holomorphic function $\phi_{g}$ such that the map

$$
U_{g} F(z)=\phi_{g}(z) F\left(g^{-1} \cdot z\right)
$$

is unitary on $\mathcal{H} L^{2}(\mathbb{D}, \alpha)$.

Then $\mathcal{H} L^{2}(\mathbb{D}, \alpha)$ is holomorphically equivalent to one of the weighted Bergman spaces.

The proof is a starred exercise. The proof is similar to that of Theorem 14, except that you need to think "hyperbolically." This means that you should express things in terms of the hyperbolic volume measure $\left(1-|z|^{2}\right)^{-2} d z$, which is invariant under the action of $S U(1,1)$, and in terms of the hyperbolic Laplacian, $\Delta_{H}=\left(1-|z|^{2}\right)^{2} \Delta$, which commutes with the action of $S U(1,1)$. You may assume these properties of the hyperbolic volume measure and the hyperbolic Laplacian. 


\subsection{Exercises}

Exercise 8 Let $U$ be $a$ an open, simply connected set in $\mathbb{C}^{1}$, and let $\alpha$ be a strictly positive smooth function on $U$. Show that there exists a holomorphic function $\phi$ with $|\phi|^{2}=\alpha$ if and only if $\log \alpha$ is harmonic.

Exercise 9 Prove Theorem 14, using the hints given after the statement of the theorem.

Exercise $10{ }^{*}$ Prove Theorem 15, using the hints given after the statement of the theorem.

\section{Canonical commutation relations}

\subsection{The standard form of the canonical commutation re- lations}

Let us make a brief digression from things holomorphic to consider the matter of the "canonical commutation relations," which Bargmann used to derive the Segal-Bargmann transform. The transform itself will make its entrance in the next section. So let us consider the Hilbert space $L^{2}(\mathbb{R}, d x)$, and two (unbounded) linear operators on it, denoted $X$ and $P$ and given by

$$
\begin{aligned}
& X f(x)=x f(x) \\
& P f(x)=-i \hbar \frac{d f}{d x} .
\end{aligned}
$$

Here $\hbar$ (pronounced "aitch-bar") is Planck's constant, which is a positive constant. We will say more about $\hbar$ in Section 7 . Note that $X$ and $P$ are not defined on all of $L^{2}(\mathbb{R}, d x)$, since $L^{2}$ functions are not necessarily differentiable, and since $x f(x)$ may not be in $L^{2}$, even if $f(x)$ is. Thus properly speaking $X$ and $P$ are defined on certain domains, which are dense subspaces of $L^{2}$. However, I am not going to worry (much) about such domain issues here, since I want to convey the basic ideas without becoming bogged down in functional-analytic technicalities. Ignoring domain issues, it is easily seen that $X$ and $P$ are selfadjoint operators on $L^{2}(\mathbb{R}, d x)$. (The classic book of Reed and Simon [RS is a good place to start on such matters.)

Let us now compute the commutator of $X$ and $P$. (Recall that the commutator of two operators $A$ and $B$, denoted $[A, B]$, is defined by $[A, B]=A B-B A$. The commutator measures the extent to which $A$ and $B$ fail to commute.) So

$$
\begin{aligned}
{[X, P] f } & =x\left(-i \hbar \frac{d f}{d x}\right)+i \hbar \frac{d}{d x}(x f(x)) \\
& =-i \hbar x \frac{d f}{d x}+i \hbar\left(\frac{d x}{d x} f(x)+x \frac{d f}{d x}\right) \\
& =i \hbar f(x) .
\end{aligned}
$$


That is,

$$
[X, P]=i \hbar I,
$$

where $I$ is the identity operator. The relation (8) is called the canonical commutation relation, abbreviated CCR. The quantum mechanical idea behind this relation will be explained in Section 7. The operator $X$ is called the position operator and the operator $P$ is called the momentum operator, for reasons to be explained in Section \&. (Of course, we know that CCR stands for Creedence Clearwater Revival, but we will allow it also to stand for canonical commutation relation.)

There are position and momentum operators in $L^{2}\left(\mathbb{R}^{d}, d x\right), X_{k}$ and $P_{k}$, $k=1, \cdots, d$, given by

$$
\begin{aligned}
X_{k} f(x) & =x_{k} f(x) \\
P_{k} f(x) & =-i \hbar \frac{\partial f}{\partial x_{k}},
\end{aligned}
$$

where $x=\left(x_{1}, \cdots, x_{d}\right)$. The relations among these are

$$
\begin{aligned}
{\left[X_{k}, X_{l}\right] } & =0 \\
{\left[P_{k}, P_{l}\right] } & =0 \\
{\left[X_{k}, P_{l}\right] } & =i \hbar \delta_{k, l} I,
\end{aligned}
$$

where $\delta_{k, l}$ is the Kronecker delta function, given by $\delta_{k, l}=0$ if $k \neq l$ and $\delta_{k, l}=1$ if $k=l$. These are the $d$-dimensional version of the canonical commutation relations. Of course these imply that $\left[P_{l}, X_{k}\right]=-i \hbar \delta_{k, l}$.

It is convenient to re-write the canonical commutation relations in terms of the so-called creation and annihilation operators (also called raising and lowering operators) defined by

$$
\begin{aligned}
& a_{k}=\frac{X_{k}+i P_{k}}{\sqrt{2}} \\
& a_{k}^{*}=\frac{X_{k}-i P_{k}}{\sqrt{2}} .
\end{aligned}
$$

Note that since $X_{k}$ and $P_{k}$ are self-adjoint, then $a_{k}^{*}$ is the adjoint of $a_{k}$, which is what the star is supposed to mean. We compute that

$$
\begin{aligned}
{\left[a_{k}, a_{l}\right] } & =\frac{1}{2}\left[X_{k}+i P_{k}, X_{l}+i P_{l}\right] \\
& =\frac{1}{2}\left(\left[X_{k}, X_{l}\right]+i\left[P_{k}, X_{l}\right]+i\left[X_{k}, P_{l}\right]-\left[P_{k}, P_{l}\right]\right) \\
& =\frac{1}{2}\left(0+\hbar \delta_{k, l} I-\hbar \delta_{k, l} I+0\right)=0,
\end{aligned}
$$


and similarly that $\left[a_{k}^{*}, a_{l}^{*}\right]=0$. Meanwhile,

$$
\begin{aligned}
{\left[a_{k}, a_{l}^{*}\right] } & =\frac{1}{2}\left[X_{k}+i P_{k}, X_{l}-i P_{l}\right] \\
& =\frac{1}{2}\left(\left[X_{k}, X_{l}\right]+i\left[P_{k}, X_{l}\right]-i\left[X_{k}, P_{l}\right]+\left[P_{k}, P_{l}\right]\right) \\
& =\frac{1}{2}\left(0+\hbar \delta_{k, l} I+\hbar \delta_{k, l} I+0\right)=\hbar \delta_{k, l} I .
\end{aligned}
$$

So the canonical commutation relations take the form

$$
\begin{aligned}
{\left[a_{k}, a_{l}\right] } & =0 \\
{\left[a_{k}^{*}, a_{l}^{*}\right] } & =0 \\
{\left[a_{k}, a_{l}^{*}\right] } & =\hbar \delta_{k, l} I .
\end{aligned}
$$

Sometimes only the third of these is written, with other commutators being understood to be zero. I have defined the creation and annihilation operators in a slightly unconventional way by not absorbing the $\hbar$ into the definition of the operators. That is, conventionally the $\sqrt{2}$ in the denominator of $a$ and $a^{*}$ is replaced by $\sqrt{2 \hbar}$. This would eliminate the factor of $\hbar$ in the CCRs. I would prefer to dispense as well with the factor of $\sqrt{2}$ in the denominator, for reasons of my own, but I don't want to break too much with tradition.

We now turn to the famous Stone-von Neumann Theorem, which explains the significance of these canonical commutation relations. The idea is to consider any Hilbert space $H$ and any collection $\left\{a_{1}, \cdots, a_{d}\right\}$ of operators, together with their adjoints $\left\{a_{1}^{*}, \cdots, a_{d}^{*}\right\}$, which satisfy the CCRs in the form (11). Unfortunately, the statement of this theorem usually given in physics is simply false, because of those annoying domain issues which we are ignoring. Nevertheless, I want to state the theorem in this imprecise way first, since this way is easier to grasp. But keep in mind that the actual theorem is different. See RS, Chap. VIII.5, Ex. 2] for a counter-example.

Claim 16 (Stone-von Neumann Non-Theorem) Let $H$ be a Hilbert space, let $a_{1}, \cdots, a_{d}$ be possibly unbounded operators on $H$, and let $a_{1}^{*}, \cdots, a_{d}^{*}$ be the adjoints of the $a_{k}$ 's. Suppose that

1. (CCRs) For all $k, l,\left[a_{k}, a_{l}\right]=\left[a_{k}^{*}, a_{l}^{*}\right]=0$ and $\left[a_{k}, a_{l}^{*}\right]=\hbar \delta_{k, l} I$, and

2. (Irreducibility) If $V$ is a closed subspace of $H$ which is invariant under all the $a_{k}$ 's and $a_{k}^{*}$ 's, then either $V=\{0\}$ or $V=H$.

Then there exists a unitary map (unique up to a constant) $U: H \rightarrow L^{2}\left(\mathbb{R}^{d}, d x\right)$ such that

$$
\begin{aligned}
U a_{k} U^{-1} & =\frac{X_{k}+i P_{k}}{\sqrt{2}} \\
U a_{k}^{*} U^{-1} & =\frac{X_{k}-i P_{k}}{\sqrt{2}},
\end{aligned}
$$

where $X_{k}$ and $P_{k}$ are given by (9). 
This non-theorem (we will have a real theorem momentarily) says that up to unitary equivalence there is only one irreducible representation of the CCRs. This result is important in quantum mechanics because it helps to justify the use of the Hilbert space $L^{2}\left(\mathbb{R}^{d}, d x\right)$ and the operators (9). After all, if you had any other Hilbert space with operators satisfying the CCRs (and irreducibility) it would be unitarily equivalent to $L^{2}\left(\mathbb{R}^{d}\right)$ in a way that took these operators to the standard creation and annihilation operators.

\subsection{The exponentiated form of the canonical commutation relations}

To get the correct form of the Stone-von Neumann theorem one needs to consider exponentiated operators, which are bounded, meaning that you don't have to worry about domain issues. So let $X_{k}$ and $P_{k}$ be the operators in (9), and consider $e^{i s X_{k} / \hbar}$ and $e^{i t P_{l} / \hbar}$. I won't go into the details, but these are everywheredefined unitary operators for each $s$ and $t$. These can be computed explicitly as follows

$$
\begin{aligned}
e^{i r X_{k} / \hbar} f(x) & =e^{i r x_{k} / \hbar} f(x) \\
e^{i s P_{l} / \hbar} f(x) & =f\left(x_{1}, \cdots, x_{l-1}, x_{l}+s, x_{l+1}, \cdots, x_{d}\right) .
\end{aligned}
$$

Equation (12) is clear, at least formally. To understand (13) formally, expand the right side in a Taylor series in powers of $s$ (without worrying about convergence). The terms in this expansion will precisely match the formal power series of the left side obtained by writing $e^{i s P_{l} / \hbar}=\sum_{n=0}^{\infty}\left(i s P_{l} / \hbar\right)^{n} / n$ !. Let's see what relations hold for these exponentiated operators. I will take $d=1$ for simplicity. Then

$$
e^{i r X / \hbar} e^{i s P / \hbar} f(x)=e^{i r x / \hbar} f(x+s)
$$

and

$$
\begin{aligned}
e^{i s P / \hbar} e^{i r X / \hbar} & =e^{i s P / \hbar}\left[e^{i r x / \hbar} f(x)\right] \\
& =e^{i r(x+s) / \hbar} f(x+s) \\
& =e^{i r s} e^{i r X / \hbar} e^{i s P / \hbar} f(x) .
\end{aligned}
$$

There are no domain issues here; this holds unambiguously for all $f$ in $L^{2}$.

We see that

$$
e^{i r X / \hbar} e^{i s P / \hbar}=e^{-i r s / \hbar} e^{i s P / \hbar} e^{i r X / \hbar} .
$$

In $d$ dimensions we have

$$
\begin{aligned}
e^{i r X_{k} / \hbar} e^{i s X_{l} / \hbar} & =e^{i s X_{l} / \hbar} e^{i r X_{k} / \hbar} \\
e^{i r P_{k} / \hbar} e^{i s P_{l} / \hbar} & =e^{i s P_{l} / \hbar} e^{i r P_{k} / \hbar} \\
e^{i r X_{k} / \hbar} e^{i s P_{l} / \hbar} & =e^{-i r s \delta_{k}, / \hbar} e^{i s P_{l} / \hbar} e^{i r X_{k} / \hbar}
\end{aligned}
$$


The equations (14) are the exponentiated form of the CCRs. One can formally derive the exponentiated form of the CCRs from the original form (10) (Exercise 14). However, this derivation is only formal and the conclusion is not actually correct. That is, (10) does not actually imply (14), without making additional domain assumptions. But this should be regarded as a technicality.

We may now state the correct form of the Stone-von Neumann Theorem. (A slightly different form of this is given in RS, Thm. VIII.14].)

Theorem 17 (Stone-von Neumann Theorem) Suppose $A_{1}, \cdots, A_{d}$ and $B_{1}, \cdots, B_{d}$ are (possibly unbounded) self-adjoint operators on a Hilbert space $H$ satisfying:

1. (CCRs) For all $k$ and $l$ and $r$ and $s, e^{i r A_{k} / \hbar}$ commutes with $e^{i s A_{l} / \hbar}, e^{i r B_{k} / \hbar}$ commutes with $e^{i s B_{l} / \hbar}$, and

$$
e^{i r A_{k} / \hbar} e^{i s B_{l} / \hbar}=e^{-i r s \delta_{k, l} / \hbar} e^{i s B_{l} / \hbar} e^{i r A_{k} / \hbar} .
$$

2. (Irreducibility) If $V$ is a closed subspace of $H$ invariant under $e^{i r A_{k} / \hbar}$ and $e^{i s B_{l} / \hbar}$ for all $k$ and $l$ and $s$ and $t$, then $V=\{0\}$ or $V=H$.

Then there is a unitary map (unique up to a constant) $U: H \rightarrow L^{2}\left(\mathbb{R}^{d}, d x\right)$ such that

$$
\begin{aligned}
U e^{i r A_{k} / \hbar} U^{-1} & =e^{i r X_{k} / \hbar} \\
U e^{i s B_{l} / \hbar} U^{-1} & =e^{i s P_{l} / \hbar},
\end{aligned}
$$

where $e^{i r X_{k} / \hbar}$ and $e^{i s P_{l} / \hbar}$ are given by $(19)$ and $(15)$.

\section{$5.3 \quad$ Exercises}

Exercise 11 Let $\tilde{X}_{k}=P_{k}$ and $\tilde{P}_{k}=-X_{k}$. Show that $\tilde{X}_{k}$ and $\tilde{P}_{k}$ satisfy the CCRs.

Exercise 12 In the notation of the previous exercise, describe the unitary transformation $U: L^{2}\left(\mathbb{R}^{d}\right) \rightarrow L^{2}\left(\mathbb{R}^{d}\right)$ such that $U \tilde{X}_{k} U^{-1}=X_{k}$ and $U \tilde{P}_{k} U^{-1}=P_{k}$.

Exercise 13 Suppose $A$ and $B$ are $n \times n$ matrices, for some (finite) number $n$. Suppose that

$$
[A, B]=c I
$$

for some constant c. Show that c must equal zero. So the CCRs cannot hold for matrices. 
Exercise $14 *$ a) Suppose that $A$ and $B$ are $n \times n$ matrices such that

$$
[A,[A, B]]=[B,[A, B]]=0 .
$$

(That is, $A$ and $B$ commute with their commutator.) Show that

$$
e^{A} e^{B}=e^{A+B+\frac{1}{2}[A, B]} .
$$

Hint: Show that $e^{s A} e^{s B} e^{-s^{2}[A, B] / 2}$ satisfies the same ordinary differential equation as $e^{s(A+B)}$.

b) If $A$ and $B$ are as in (a) show that

$$
e^{i r A / \hbar} e^{i s B / \hbar}=e^{-i r s / \hbar} e^{i s B / \hbar} e^{i r A / \hbar} .
$$

c) Explain why this gives a formal argument that the CCRs in form (10) should imply the exponentiated CCRs (14).

Of course, (19) does not actually imply (14) without additional domain conditions.

Exercise 15 * This exercise guides you through a formal argument for the conventional (non-theorem) form of Stone-von Neumann. (This argument cannot be made rigorous without additional domain assumptions.) We will consider only $d=1$, though the general case is nearly the same. In all cases, "show" should be understood as "show ignoring domain issues." So let $H$ be any Hilbert space and let $a$ and $a^{*}$ satisfy the conditions of Claim 10 .

a) Show that $E:=a^{*} a$ is self-adjoint and positive.

b) Let $v \neq 0$ be any eigenvector for $E$ with eigenvalue $\lambda$. Show that

$$
\text { Eav }=(\lambda-1) a v .
$$

c) Show that there exists $k \geq 0$ such that $a^{k} v \neq 0$ but $a^{k+1} v=0$. Hint: $E$ is positive.

d) Let $u=a^{k} v$, so that $u \neq 0$ but au $=0$ and so also $E u=0$. Show inductively that

$$
E\left(a^{*}\right)^{n} u=n\left(a^{*}\right)^{n} u, \quad n=0,1, \cdots .
$$

e) Show inductively that

$$
a\left(a^{*}\right)^{n} u=n\left(a^{*}\right)^{n-1} u, \quad n=0,1, \cdots .
$$

f) Show that

$$
\begin{aligned}
\left\langle\left(a^{*}\right)^{n} u,\left(a^{*}\right)^{n} u\right\rangle & =n !\langle u, u\rangle, \quad n=0,1, \cdots \\
\left\langle\left(a^{*}\right)^{n} u,\left(a^{*}\right)^{m} u\right\rangle & =0, \quad n \neq m .
\end{aligned}
$$

g) Show that the closed span of $\left\{\left(a^{*}\right)^{n} u\right\}$ is all of $H$.

$h)$ If $\left(K, b, b^{*}\right)$ is any other irreducible representation of the CCRs, show that $K$ is unitarily equivalent to $H$. Hint: let $\tilde{u}$ be the analog of $u$ in $K$ (chosen to have the same norm as $u)$. Define $U: K \rightarrow H$ so that $U \tilde{u}=u$ and $U\left(b^{*}\right)^{n} \tilde{u}=$ $\left(a^{*}\right)^{n} u$. 


\section{The Segal-Bargmann transform}

\subsection{Bargmann's extension of Fock's observation}

Let us consider for now just the $d=1$ case of the CCRs. Fock (1928) made the following observation. Consider the space $\mathcal{H}(\mathbb{C})$ of holomorphic functions on $\mathbb{C}$. Consider the operators $z$ and $\hbar d / d z$ on $\mathcal{H}(\mathbb{C})$, where $z$ denotes multiplication by $z$. Fock observed that

$$
\begin{aligned}
{\left[\hbar \frac{d}{d z}, z\right] f } & =\hbar \frac{d}{d z}(z f(z))-\hbar z \frac{d f}{d z} \\
& =\hbar f(z)+\hbar z \frac{d f}{d z}(z)-\hbar z \frac{d f}{d z} \\
& =\hbar f(z) .
\end{aligned}
$$

That is,

$$
\left[\hbar \frac{d}{d z}, z\right]=\hbar I
$$

Thus $\hbar d / d z$ and multiplication by $z$ have the same commutation relations as the annihilation and creation operators.

However, this does not constitute a representation of the canonical commutation relations. After all, the CCRs require that we have a Hilbert space $H$ and operators $a$ and $a^{*}$ that are adjoints of one another satisfying $\left[a, a^{*}\right]=\hbar I$. Bargmann in [B1] sought an inner product on $\mathcal{H}(\mathbb{C})$ which would make $z$ and $\hbar d / d z$ adjoints of one another. It is not too hard to work out what such an inner product would have to be (see [B1]); it turns out to be the inner product on the Segal-Bargmann space $\mathcal{H} L^{2}\left(\mathbb{C}, \mu_{\hbar}\right)$. Note here that we are identifying the parameter $t$ in the Segal-Bargmann space with Planck's constant $\hbar$. So we have the following result, stated as usual without specifying domains. (See [B1] for a precise statement.)

Theorem 18 In the Segal-Bargmann space $\mathcal{H} L^{2}\left(\mathbb{C}^{d}, \mu_{\hbar}\right)$,

$$
\left(z_{k}\right)^{*}=\hbar \frac{\partial}{\partial z_{k}}
$$

where $z_{k}$ denotes multiplication by $z_{k}$ and ${ }^{*}$ denotes the adjoint with respect to the inner product on $\mathcal{H} L^{2}\left(\mathbb{C}^{d}, \mu_{\hbar}\right)$.

Remark. In the theory of spherical harmonics one needs an inner product on the space $\mathcal{P}\left(\mathbb{R}^{d}\right)$ of polynomials in $\mathbb{R}^{d}$ with the property that $\left\langle\left(x_{1}^{2}+\cdots x_{d}^{2}\right) p, q\right\rangle=$ $\langle p, \Delta q\rangle$ for all polynomials $p$ and $q$. Such an inner product is obtained by analytically continuing $p$ and $q$ to $\mathbb{C}^{d}$ and then using the inner product on $L^{2}\left(\mathbb{C}^{d}, \mu_{1}\right)$.

Proof (modulo domain issues). An alternative proof is given in Exercise 16. We will for notational simplicity consider only the $d=1$ case. Recall the definition of the operators $\partial / \partial z$ and $\partial / \partial \bar{z}$, acting on not-necessarily-holomorphic 
functions on $\mathbb{C}$ :

$$
\begin{aligned}
\frac{\partial}{\partial z} & =\frac{1}{2}\left(\frac{\partial}{\partial x}-i \frac{\partial}{\partial y}\right) \\
\frac{\partial}{\partial \bar{z}} & =\frac{1}{2}\left(\frac{\partial}{\partial x}+i \frac{\partial}{\partial y}\right)
\end{aligned}
$$

A $\mathcal{C}^{1}$ function on $\mathbb{C}$ is holomorphic if and only if $\partial f / \partial \bar{z}=0$. (This is equivalent to the Cauchy-Riemann equations.) If $f$ is holomorphic, then the usual complex derivative $d f / d z$ coincides with $\partial f / \partial z$.

So now assume that $F$ and $G$ are in $\mathcal{H} L^{2}\left(\mathbb{C}, \mu_{\hbar}\right)$, and consider

$$
\begin{aligned}
\left\langle\frac{\partial F}{\partial z}, G\right\rangle & =\frac{1}{2} \int_{\mathbb{C}} \overline{\left(\frac{\partial F}{\partial x}-i \frac{\partial F}{\partial y}\right)} G(z) \mu_{\hbar}(z) d z \\
& =\frac{1}{2} \int_{\mathbb{C}}\left(\frac{\partial \bar{F}}{\partial x}+i \frac{\partial \bar{F}}{\partial y}\right) G(z) \mu_{\hbar}(z) d z .
\end{aligned}
$$

We now want to integrate by parts. I will assume that $F$ and $G$ grow slowly enough at infinity that the rapid decay of $\mu_{\hbar}$ will kill off the boundary terms in the integration by parts. In that case we get

$$
\begin{aligned}
\left\langle\frac{\partial F}{\partial z}, G\right\rangle & =-\frac{1}{2} \int_{\mathbb{C}} \bar{F}(z)\left(\frac{\partial}{\partial x}+i \frac{\partial}{\partial y}\right)\left(G(z) \mu_{\hbar}(z)\right) d z \\
& =-\int_{\mathbb{C}} \bar{F}(z) \frac{\partial}{\partial \bar{z}}\left(G(z) \mu_{\hbar}(z)\right) d z \\
& =-\int_{\mathbb{C}} \bar{F}(z) \frac{\partial G}{\partial \bar{z}} \mu_{\hbar}(z) d z-\int_{\mathbb{C}} \bar{F}(z) G(z) \frac{\partial \mu_{\hbar}(z)}{\partial \bar{z}} d z
\end{aligned}
$$

Now, since $G$ is holomorphic, $\partial G / \partial \bar{z}=0$. Meanwhile we compute that

$$
\begin{aligned}
\frac{\partial \mu_{\hbar}}{\partial \bar{z}} & =\frac{\partial}{\partial \bar{z}}(\pi \hbar)^{-1} e^{-z \bar{z} / \hbar} \\
& =\left(-\frac{z}{\hbar}\right)(\pi \hbar)^{-1} e^{-z \bar{z} / \hbar}
\end{aligned}
$$

So we get just

$$
\begin{aligned}
\left\langle\frac{\partial F}{\partial z}, G\right\rangle & =\int_{\mathbb{C}} \bar{F}(z) G(z) \frac{z}{\hbar} \mu_{\hbar}(z) d z \\
& =\frac{1}{\hbar}\langle F, z G\rangle .
\end{aligned}
$$

That is, $(\partial / \partial z)^{*}=(1 / \hbar) z$, or equivalently, $(z)^{*}=\hbar \partial / \partial z$.

So the canonical commutation relations hold with $a=\hbar \partial / \partial z$ and $a^{*}=z$. If we assume irreducibility and that the exponentiated form of the CCRs hold, then the Stone-von Neumann theorem will tell us that there is a unitary map (unique up to a constant) from $\mathcal{H} L^{2}\left(\mathbb{C}, \mu_{\hbar}\right)$ to $L^{2}(\mathbb{R}, d x)$ which turns these 
operators into the standard creation and annihilation operators. Such a map does indeed exist and is the Segal-Bargmann transform, which we consider in the next subsection. In fact, it is not hard to show that the exponentiated CCRs do hold-see Exercise 17. The exponentiated position and momentum operators are expressed in terms of the unitarized translation operators $T_{a}$.

We can easily extend this analysis to the $d$-dimensional case, by considering the Segal-Bargmann space $\mathcal{H} L^{2}\left(\mathbb{C}^{d}, \mu_{\hbar}\right)$ and considering the operators $z_{k}$ (multiplication by $z_{k}$ ) and $\hbar \partial / \partial z_{k}$.

\subsection{The transform}

The calculations of the previous subsection suggest (almost prove) that there should be a unitary map $A_{\hbar}: L^{2}\left(\mathbb{R}^{d}, d x\right) \rightarrow \mathcal{H} L^{2}\left(\mathbb{C}^{d}, \mu_{\hbar}\right)$ that intertwines the usual creation and annihilation operators with the operators $z_{k}$ and $\hbar d / d z_{k}$. There is indeed such a map, as described in the following theorem.

Theorem 19 Consider the map $A_{\hbar}: L^{2}\left(\mathbb{R}^{d}, d x\right) \rightarrow \mathcal{H}\left(\mathbb{C}^{d}, \mu_{\hbar}\right)$ given by

$$
A_{\hbar} f(z)=(\pi \hbar)^{-d / 4} \int_{\mathbb{R}^{d}} e^{\left(-z^{2}+2 \sqrt{2} x \cdot z-x^{2}\right) / 2 \hbar} f(x) d x .
$$

1. For all $f \in L^{2}\left(\mathbb{R}^{d}, d x\right)$, the integral is convergent and is a holomorphic function of $z \in \mathbb{R}^{d}$.

2. The map $A_{\hbar}$ is a unitary map of $L^{2}\left(\mathbb{R}^{d}, d x\right)$ onto $\mathcal{H} L^{2}\left(\mathbb{C}^{d}, \mu_{\hbar}\right)$.

3. For $k=1, \cdots, d$

$$
\begin{aligned}
& A_{\hbar}\left(\frac{X_{k}+i P_{k}}{\sqrt{2}}\right) A_{\hbar}^{-1}=\hbar \frac{\partial}{\partial z_{k}} \\
& A_{\hbar}\left(\frac{X_{k}-i P_{k}}{\sqrt{2}}\right) A_{\hbar}^{-1}=z_{k} .
\end{aligned}
$$

There are many different ways to prove this theorem. However, it is reasonable to prove it in a way that makes use of the canonical commutation relations. At the same time, we do not want to rely on the "non-theorem" form of the Stone-von Neumann theorem, and we do not want to have to check irreducibility, which we would need to do to make use of either form of Stone-von Neumann. So we will follow the following strategy. First, we verify that the intertwining formulas (16) and (17) hold at least on "nice" functions. Second, we use (16) and (17) to show that $A_{\hbar}$ maps a known orthonormal basis for $L^{2}\left(\mathbb{R}^{d}\right)$ onto a known orthonormal basis for $\mathcal{H} L^{2}\left(\mathbb{C}^{d}, \mu_{\hbar}\right)$. (See also the argument in [B1], which is a very readable paper. Bargmann normalizes $\hbar$ to be 1 .)

Proof of Theorem 19. As usual we will do the proof in the case $d=1$, though the general case is entirely analogous. The integral converges because the function $e^{-\left(z^{2}+2 \sqrt{2} x z-x^{2}\right) / 2 \hbar}$ is square-integrable as a function of $x$ for each fixed $z$. Holomorphicity can be proved using Morera's Theorem. 
We do not yet know that $A_{\hbar}$ is invertible. So we will prove (16) and (17) in the form

$$
\begin{aligned}
& A_{\hbar} \frac{X+i P}{\sqrt{2}}=\hbar \frac{\partial}{\partial z} A_{\hbar} \\
& A_{\hbar} \frac{X-i P}{\sqrt{2}}=z A_{\hbar} .
\end{aligned}
$$

I will assume that we apply $A_{\hbar}$ only to "nice" functions $f$, namely ones that are smooth and decay rapidly at infinity, so that we may freely integrate by parts and differentiate under the integral sign.

Then we compute that

$$
\begin{aligned}
\frac{d}{d z}\left(A_{\hbar} f\right)(z) & =(\pi \hbar)^{-1 / 4} \int_{\mathbb{R}} \frac{d}{d z} e^{\left(-z^{2}+2 \sqrt{2} x z-x^{2}\right) / 2 \hbar} f(x) d x \\
& =(\pi \hbar)^{-1 / 4} \int_{\mathbb{R}}\left(-\frac{z}{\hbar}+\frac{\sqrt{2} x}{\hbar}\right) e^{\left(-z^{2}+2 \sqrt{2} x z-x^{2}\right) / 2 \hbar} f(x) d x \\
& =-\frac{z}{\hbar} A_{\hbar} f(z)+\frac{\sqrt{2}}{\hbar} A_{\hbar}[x f(x)](z) .
\end{aligned}
$$

So

$$
\frac{d}{d z} A_{\hbar}=-\frac{z}{\hbar} A_{\hbar}+\frac{\sqrt{2}}{\hbar} A_{\hbar} X,
$$

where as usual $X$ means multiplication by $x$. Next compute using integration by parts:

$$
\begin{aligned}
A_{\hbar}\left[\frac{d f}{d x}\right](z) & =(\pi \hbar)^{-1 / 4} \int_{\mathbb{R}} e^{\left(-z^{2}+2 \sqrt{2} x z-x^{2}\right) / 2 \hbar} \frac{d f}{d x} d x \\
& =-(\pi \hbar)^{-1 / 4} \int_{\mathbb{R}}\left(\frac{\sqrt{2} z}{\hbar}-\frac{x}{\hbar}\right) e^{\left(-z^{2}+2 \sqrt{2} x z-x^{2}\right) / 2 \hbar} f(x) d x,
\end{aligned}
$$

which tells us that

$$
A_{\hbar} \frac{d}{d x}=-\frac{\sqrt{2}}{\hbar} z A_{\hbar}+\frac{1}{\hbar} A_{\hbar} X .
$$

We may solve (20) for $z A_{\hbar}$ to get one part of (18), and then substitute this expression for $z A_{\hbar}$ into (19) and solve for $(d / d z) A_{\hbar}$ to get the other part of (18). The algebra is left to the reader.

Having established (18), we now prove the unitarity of $A_{\hbar}$ in a way that is similar to Exercise 15 of the previous section. Consider the "ground state" function

$$
f_{0}(x)=(\pi \hbar)^{-1 / 4} e^{-x^{2} / 2 \hbar} .
$$


This is the unique (up to a constant) function with the property that $a f_{0}=0$, where $a=2^{-1 / 2}(x+\hbar d / d x)$ is the annihilation operator. Applying the SegalBargmann operator $A_{\hbar}$ to $f_{0}$ we get with a little algebra

$$
\begin{aligned}
\left(A_{\hbar} f_{0}\right)(z) & =(\pi \hbar)^{-1 / 2} \int_{\mathbb{R}} e^{-(z-\sqrt{2} x)^{2} / 2 \hbar} d x \\
& =1 .
\end{aligned}
$$

That is, $A_{\hbar} f_{0}$ is the constant function 1. To evaluate (21) first observe that for $z \in \mathbb{R}$, a change of variable shows that the integral is independent of $z$. Since $A_{\hbar} f_{0}$ is holomorphic, if it is constant on $\mathbb{R}$ then it is constant on $\mathbb{C}$. The evaluation of the constant is a standard Gaussian integral.

Once it is established that $A_{\hbar} f_{0}=\mathbf{1}$, the intertwining properties (18) show that

$$
A_{\hbar}\left(\left(a^{*}\right)^{n} f_{0}\right)=z^{n} \mathbf{1}=z^{n} .
$$

But the functions $\left(a^{*}\right)^{n} f_{0}$ are the Hermite functions, which are known to form an orthogonal basis for $L^{2}(\mathbb{R})$, with $\left\|\left(a^{*}\right)^{n} f_{0}\right\|_{L^{2}(\mathbb{R})}^{2}=\hbar^{n} n$ !. (That they are orthogonal with the indicated norms is proved using the canonical commutation relations as in Exercise 15 of the previous section.) Meanwhile, we computed in Section 3 that the functions $z^{n}$ form an orthogonal basis for $\mathcal{H} L^{2}\left(\mathbb{C}, \mu_{\hbar}\right)$ with $\left\|z^{n}\right\|^{2}=\hbar^{n} n$ !. Since $A_{\hbar}$ takes an orthogonal basis to an orthogonal basis with the same norms, $A_{\hbar}$ is unitary.

I now want to describe a slightly different form of the Segal-Bargmann transform, obtained by making the "ground state transformation." This transformation is necessary if one is going to take the infinite-dimensional limit (as in Segal), and is often convenient even in finite dimensions. Consider the unitary map

$$
G_{\hbar}: L^{2}\left(\mathbb{R}^{d}, d x\right) \rightarrow L^{2}\left(\mathbb{R}^{d},(\pi \hbar)^{-d / 2} e^{-x^{2} / \hbar} d x\right)
$$

given by

$$
G_{\hbar} f(x)=\frac{f(x)}{f_{0}(x)}=\frac{f(x)}{(\pi \hbar)^{-d / 4} e^{-x^{2} / 2 \hbar}},
$$

where $f_{0}(x):=(\pi \hbar)^{-d / 4} e^{-x^{2} / 2 \hbar}$ is the ground state in $\mathbb{R}^{d}$. Note that the measure in the image space is the measure $f_{0}(x)^{2} d x$, and that the unitarity of $G_{\hbar}$ is a very elementary calculation. This is called the ground state transformation since we are dividing each function by the ground state $f_{0}$. Note that $G_{\hbar} f_{0}$ is the constant function $\mathbf{1}$. So the effect of $G_{\hbar}$ is to turn the ground state into the constant function $\mathbf{1}$ and to convert from Lebesgue measure to the measure $f_{0}(x)^{2} d x$. 
I leave it as an exercise to calculate that

$$
\begin{aligned}
G a_{k} G^{-1} & =\frac{\hbar}{\sqrt{2}} \frac{\partial}{\partial x_{k}} \\
G a_{k}^{*} G^{-1} & =\sqrt{2} x_{k}-\frac{\hbar}{\sqrt{2}} \frac{\partial}{\partial x_{k}} .
\end{aligned}
$$

It is now convenient to make an additional change of variable by letting $y=$ $\sqrt{2} x$, and then renaming our variable back to $x$. The resulting creation and annihilation operators then take the form

$$
\begin{aligned}
& \tilde{a}_{k}=\hbar \frac{\partial}{\partial x_{k}} \\
& \tilde{a}_{k}^{*}=x_{k}-\hbar \frac{\partial}{\partial x_{k}} .
\end{aligned}
$$

Our Hilbert space becomes (after this change) $L^{2}\left(\mathbb{R}^{d}, \rho_{\hbar}(x) d x\right)$, where

$$
\rho_{\hbar}(x)=(2 \pi \hbar)^{-d / 2} e^{-x^{2} / 2 \hbar} .
$$

Our goal, then, is to find a unitary map from $L^{2}\left(\mathbb{R}^{d}, \rho_{\hbar}(x) d x\right)$ to $\mathcal{H} L^{2}\left(\mathbb{C}^{d}, \mu_{\hbar}\right)$ which will convert the operators in (23) to the operators $\hbar \partial / \partial z_{k}$ and $z_{k}$. This operator can be obtained by undoing the change of variable $x \rightarrow \sqrt{2} x$ and the ground state transformation, and then applying the Segal-Bargmann transform $A_{\hbar}$. I will spare the reader the calculation and simply record the result. Alternatively, one can prove Theorem 20 directly in a way similar to the proof of Theorem 19. See Exercise 19.

Theorem 20 For all $\hbar>0$, consider the map $B_{\hbar}: L^{2}\left(\mathbb{R}^{d}, \rho_{\hbar}(x) d x\right) \rightarrow$ $\mathcal{H}\left(\mathbb{C}^{d}\right)$ given by

$$
\begin{aligned}
B_{\hbar} f(z) & =\int_{\mathbb{R}^{d}} \rho_{\hbar}(z-x) f(x) d x \\
& =(2 \pi \hbar)^{-d / 2} \int_{\mathbb{R}^{d}} e^{-(z-x)^{2} / 2 \hbar} f(x) d x .
\end{aligned}
$$

1. For all $f \in L^{2}\left(\mathbb{R}^{d}, \rho_{\hbar}(x) d x\right)$ this integral is absolutely convergent and gives a holomorphic function of $z \in \mathbb{C}^{d}$.

2. The map $B_{\hbar}$ is a unitary map of $L^{2}\left(\mathbb{R}^{d}, \rho_{\hbar}(x) d x\right)$ onto $\mathcal{H} L^{2}\left(\mathbb{C}^{d}, \mu_{\hbar}\right)$.

3. For all $k=1, \cdots, d$

$$
\begin{aligned}
& B_{\hbar} \tilde{a}_{k} B_{\hbar}^{-1}=\hbar \frac{\partial}{\partial z_{k}} \\
& B_{\hbar} \tilde{a}_{k}^{*} B_{\hbar}^{-1}=z_{k},
\end{aligned}
$$

where $\tilde{a}_{k}$ and $\tilde{a}_{k}^{*}$ are given by (23). 
Remarks. 1) This form of the Segal-Bargmann transform is very close to the finite-dimensional version of what Segal described in [53, Thm. 5] (or [BSZ, Thm. 1.14]). (See Exercise 20.) The only differences are that Segal uses antiholomorphic instead of holomorphic functions, and that he does not make the change of variable $y=\sqrt{2} x$, so that there are some factors of $\sqrt{2}$ left in his formulas.

2) I have made the change of variable $y=\sqrt{2} x$ so that the transform will have the form of a convolution, namely, an integral of $f(x)$ against a function of $z-x$, as in (24). Although this change is only a convenience in the $\mathbb{R}^{d}$ case, it is necessary to a generalization of the Segal-Bargmann transform to compact Lie groups, as described in Section 9.

The unitarity of the Segal-Bargmann transform (in either the $A_{\hbar}$ or $B_{\hbar}$ form) can be used to give yet another derivation of the reproducing kernel for the Segal-Bargmann space. See Exercise 22.

\subsection{The "invariant" form of the Segal-Bargmann trans- form}

I wish now to describe briefly another form of the Segal-Bargmann transform that is technically advantageous. It expresses the transform as a convolution as in the $B_{\hbar}$ form but has as its domain Hilbert space $L^{2}\left(\mathbb{R}^{d}, d x\right)$. Define a density $\nu_{\hbar}$ on $\mathbb{C}^{d}$ by

$$
\nu_{\hbar}(z)=(\pi \hbar)^{-d / 2} e^{-(\operatorname{Im} z)^{2} / \hbar} .
$$

We then have the associated holomorphic function space $\mathcal{H} L^{2}\left(\mathbb{C}^{d}, \nu_{\hbar}\right)$. Recall the density $\rho_{\hbar}$ from the previous subsection given by

$$
\rho_{\hbar}(x)=(2 \pi \hbar)^{-d / 2} e^{-x^{2} / 2 \hbar} .
$$

Observe that this function admits an entire analytic continuation to $\mathbb{C}^{d}$, also called $\rho_{\hbar}$.

Theorem 21 Consider the map $C_{\hbar}: L^{2}\left(\mathbb{R}^{d}, d x\right) \rightarrow \mathcal{H} L^{2}\left(\mathbb{C}^{d}, \nu_{\hbar}\right)$ given by

$$
\begin{aligned}
C_{\hbar} f(z) & =\int_{\mathbb{R}^{d}} \rho_{\hbar}(z-x) f(x) d x \\
& =(2 \pi \hbar)^{-d / 2} \int_{\mathbb{R}^{d}} e^{-(z-x)^{2} / 2 \hbar} f(x) d x .
\end{aligned}
$$

1. For all $f \in L^{2}\left(\mathbb{R}^{d}, d x\right)$ the integral defining $C_{\hbar} f(z)$ is absolutely convergent and holomorphic in $z \in \mathbb{C}^{d}$.

2. The map $C_{\hbar}$ is a unitary map of $L^{2}\left(\mathbb{R}^{d}, d x\right)$ onto $\mathcal{H} L^{2}\left(\mathbb{C}^{d}, \nu_{\hbar}\right)$.

3. For $k=1, \cdots, d$,

$$
\begin{aligned}
& C_{\hbar}\left(X_{k}-i P_{k}\right) C_{\hbar}^{-1}=z_{k} \\
& C_{\hbar}\left(X_{k}+i P_{k}\right) C_{\hbar}^{-1}=z_{k}+2 \hbar \frac{\partial}{\partial z_{k}}
\end{aligned}
$$


where $z_{k}$ denotes multiplication by $z_{k}$.

Remarks. 1) This form of the Segal-Bargmann transform is not truly different from the conventional forms, just a convenient alternative normalization. See Exercise 21. In fact, we can relate, say, the $A_{\hbar}$ and $C_{\hbar}$ forms of the transform as follows:

$$
\left[C_{\hbar} f\right](z)=(4 \pi \hbar)^{-d / 4} e^{-z^{2} / 4 \hbar}\left[A_{\hbar} f\right]\left(\frac{z}{\sqrt{2}}\right) .
$$

This is by direct calculation using the formulas defining $A_{\hbar}$ and $C_{\hbar}$. I will not prove the above theorem, since it is similar to the proofs for the $A_{\hbar}$ and $B_{\hbar}$ forms of the transform. One can also deduce the unitarity of $C_{\hbar}$ from that of $A_{\hbar}$ using the relation (26).

2) The formula for $C_{\hbar}$ is precisely the same as that for $B_{\hbar}$. However, when considering $B_{\hbar}$ we were using different measures on both the $\mathbb{R}^{d}$ side and the $\mathbb{C}^{d}$ side.

3) I am considering a modified set of creation and annihilation operators $\hat{a}_{k}^{*}=$ $X_{k}-i P_{k}$ and $\hat{a}_{k}=X_{k}+i P_{k}$, without the usual factors of $\sqrt{2}$ in the denominator. In $\mathcal{H} L^{2}\left(\mathbb{C}^{d}, \nu_{\hbar}\right)$ these correspond to the operators $z_{k}$ and $z_{k}+2 \hbar \partial / \partial z_{k}$. These satisfy the relation

$$
\left[X_{k}+i P_{k}, X_{l}-i P_{l}\right]=2 \hbar \delta_{k, l}
$$

and correspondingly

$$
\left[z_{k}+2 \hbar \frac{\partial}{\partial z_{k}}, z_{l}\right]=2 \hbar \delta_{k, l},
$$

as is easily calculated. Also, in $\mathcal{H} L^{2}\left(\mathbb{C}^{d}, \nu_{\hbar}\right)$ the operators $z_{k}$ and $z_{k}+2 \hbar \partial / \partial z_{k}$ are adjoints of one another, as I invite you to verify.

4) There is an unfortunate minus sign in this business, namely that the operator $z_{k}$ corresponds on the $\mathbb{R}^{d}$ side to $X_{k}-i P_{k}$ instead of $X_{k}+i P_{k}$. However, this is an improvement over the standard Segal-Bargmann transform, where $z_{k}$ corresponds to $\left(X_{k}-i P_{k}\right) / \sqrt{2}$. The minus sign could be fixed either by redefining the conventions of quantum mechanics or (as in Segal) working with anti-holomorphic functions instead of holomorphic ones.

\subsection{Historical remarks}

In the summer of 1960 there was a conference in Boulder, Colorado, attended by both Valentine Bargmann and Irving Segal. At this conference Segal gave a talk which described the infinite-dimensional $(d=\infty)$ version of the SegalBargmann space, but not of the transform. After Segal's talk Bargmann told Segal that he (Bargmann) had been working on a similar theory but in the finite-dimensional case. Bargmann then published a paper [B1] in 1961 in Communications on Pure and Applied Mathematics describing the finite-dimensional Segal-Bargmann space, the associated transform, and various other interesting 
results. Bargmann's 1961 paper has a footnote (p. 191) acknowledging that "the Hilbert space defined here has already been used by I.E. Segal for a representation of the quantum mechanical canonical operators," as described in Segal's 1960 talk in Boulder.

Meanwhile, Segal's work on the infinite-dimensional theory was published in the proceedings of the Boulder conference [S1], which appeared in 1963, and in a paper in the Illinois Journal of Mathematics [S2] that appeared in 1962. The paper [52] has a footnote (p. 520) acknowledging that at the Boulder meeting "Professor Bargmann informed us that in the case of systems of a finite number of degrees of freedom he had independently studied aspects of the representation." Segal's conference proceeding article does not discuss a transform. The Illinois Journal paper proves (Corollary 4.1) that the holomorphic function representation is unitarily equivalent to a real function representation, but does not describe this equivalence (which would be the Segal-Bargmann transform) explicitly.

After finishing his 1961 paper Bargmann had a serious illness. After recuperating he wrote a short paper that appeared in the Proceedings of the National Academy of Sciences (U.S.A) in 1962 [B2]. In this paper Bargmann described the infinite-dimensional version of the Segal-Bargmann space, having forgotten that Segal had already treated this case in his 1960 talks. Once Bargmann realized his mistake he published an acknowledgment [B3], noting that his oversight had resulted from "exceptional circumstances."

Finally, in 1978 Segal published a paper S3 that gives a technically better description of the Segal-Bargmann space in infinitely many degrees of freedom and describes explicitly the corresponding transform (similar to what I call $B_{\hbar}$ ). In this paper Segal cites Bargmann's 1961 paper B1 and the acknowledgment to Bargmann's 1962 paper [B2], but not the 1962 paper itself. This has fostered some confusion (reflected in the citations of other authors) as to whether the acknowledgment refers to Bargmann's 1961 paper or to his 1962 paper. See Section 10 for a description of the infinite-dimensional transform similar to that of [S3].

I should also mention John Klauder, who published a paper [K] in Annals of Physics in 1960 that described certain states (now called coherent states) and a "resolution of the identity" which is equivalent to the isometricity of the Segal-Bargmann transform. Although Klauder does not explicitly introduce either the Segal-Bargmann transform or the corresponding holomorphic function space, both of these objects are implicit in his resolution of the identity. There has been much work lately on coherent states, which are closely related to the Segal-Bargmann transform, but with a slightly different point of view. See the discussion of coherent states in Section 10.

\subsection{Exercises}

Exercise 16 This exercise gives an alternative proof of Theorem 18. We know that the functions $\psi_{n}(z):=z^{n} / \sqrt{\hbar^{n} n !}, n=0,1,2, \cdots$ form an orthonormal basis for $\mathcal{H} L^{2}\left(\mathbb{C}, \mu_{\hbar}\right)$. Given $F_{1}, F_{2} \in \mathcal{H} L^{2}\left(\mathbb{C}, \mu_{\hbar}\right)$, we can write $F_{1}(z)=$ 
$\sum a_{n} \psi_{n}(z)$ and $F_{2}(z)=\sum b_{n} \psi_{n}(z)$. Compute how $z$ and $\hbar d / d z$ act on the $\psi_{n}$ 's and use this to show that $\left\langle z F_{1}, F_{2}\right\rangle=\left\langle F_{1}, \hbar d F_{2} / d z\right\rangle$.

Exercise 17 Recall the unitarized translation operators $T_{a}$ on $\mathcal{H} L^{2}\left(\mathbb{C}, \mu_{\hbar}\right)(a \in$ $\mathbb{C})$ described in Theorem 11 (with the parameter $t$ in that theorem now called $\hbar)$.

a) A collection $\left\{U_{s}\right\}_{s \in \mathbb{R}}$ of unitary operators is called a one-parameter unitary group if $U_{0}=I$ and $U_{r+s}=U_{r} U_{s}$ for all $r, s \in \mathbb{R}$. Let

$$
\begin{aligned}
V_{r} & =T_{-i r / \sqrt{2}} \\
W_{s} & =T_{-s / \sqrt{2}} .
\end{aligned}
$$

Show that $V_{r}$ and $W_{s}$ are one-parameter unitary groups.

b) Show that

$$
V_{r} W_{s}=e^{-i r s / \hbar} W_{s} V_{r}
$$

for all $r, s \in \mathbb{R}$.

c) A standard functional-analytic result (Stone's Theorem) states that every (strongly continuous) one-parameter unitary group $U_{s}$ may be expressed uniquely in the form $U_{s}=e^{i s A / \hbar}$, where $A$ is a self-adjoint operator given (on a suitable domain) by

$$
A=-\left.i \hbar \frac{d}{d s}\right|_{s=0} U_{s}
$$

The operator $A$ is called the generator of $U_{s}$.

Show that the generator of $V_{r}$ is

$$
A=\frac{1}{\sqrt{2}}\left(\hbar \frac{d}{d z}+z\right)=\frac{1}{\sqrt{2}}\left(a+a^{*}\right)
$$

and that the generator of $W_{s}$ is

$$
B=\frac{1}{i \sqrt{2}}\left(\hbar \frac{d}{d z}-z\right)=\frac{1}{i \sqrt{2}}\left(a-a^{*}\right) .
$$

Conclude that $A$ and $B$ satisfy the exponentiated canonical commutation relations.

Exercise 18 Suppose $\psi \in L^{2}(\mathbb{R}, d x)$ satisfies a $\psi=0$, where $a=2^{-1 / 2}(x+$ $\hbar d / d x)$ is the annihilation operator. Show that $\psi(x)=c \exp \left(-x^{2} / 2 \hbar\right)$.

Exercise 19 Verify Point 3 of Theorem 20 in the case $d=1$, by imitating the proof of Theorem 19 . 
Exercise 20 Show that $B_{\hbar}$ can be computed as

$$
B_{\hbar} f(z)=\int_{\mathbb{R}^{d}} e^{-z^{2} / 2 \hbar} e^{z \cdot x / \hbar} f(x) \rho_{\hbar}(x) d x .
$$

Exercise 21 Show that $\mathcal{H} L^{2}\left(\mathbb{C}^{d}, \nu_{\hbar}\right)$ is holomorphically equivalent to $\mathcal{H} L^{2}\left(\mathbb{C}^{d}, \mu_{2 \hbar}\right)$.

Exercise $22{ }^{*}$ Regard $A_{\hbar}$ (with $d=1$ ) as an isometric map of $L^{2}(\mathbb{R}, d x)$ into the full $L^{2}$ space $L^{2}\left(\mathbb{C}, \mu_{\hbar}\right)$, whose image is the holomorphic subspace $\mathcal{H} L^{2}\left(\mathbb{C}, \mu_{\hbar}\right)$.

a) Show that $A_{\hbar}^{*} A_{\hbar}=I$ on $L^{2}(\mathbb{R}, d x)$ and that $A_{\hbar} A_{\hbar}^{*}=P$, where $P$ is the orthogonal projection from $L^{2}\left(\mathbb{C}, \mu_{\hbar}\right)$ onto $\mathcal{H} L^{2}\left(\mathbb{C}, \mu_{\hbar}\right)$.

b) Compute $A_{\hbar}^{*}$. Hint: How do you compute the adjoint of an integral operator?

c) Compute $A_{\hbar} A_{\hbar}^{*}$ and show that your formula agrees with our formula for the reproducing kernel of the Segal-Bargmann space.

\section{Quantum mechanics and quantization}

\subsection{A brief survey of classical mechanics}

See, for example, the book of Thirring [Th] for more information. We begin as usual with the one-dimensional case, which means that we consider a particle moving, say, along a wire. The motion of such a particle is governed by Newton's equation $F=m a$. This means that if $x(t)$ is the position of the particle, so that $\ddot{x}(t)$ (the second derivative with respect to time) is the acceleration, then $m \ddot{x}=F$, where $F$ is the force. (Here $m$ is the particle's mass.) Frequently $F$ depends only on position, and can be expressed in the form $F(x)=-V^{\prime}(x)$, where $V$ is the potential energy and $V^{\prime}$ denotes the derivative with respect to $x$. So our equation of motion becomes

$$
m \ddot{x}=-V^{\prime}(x) .
$$

Now, this is a second-order equation. It is convenient to re-write this as a system of two first-order equations. Let

$$
p=m \dot{x}
$$

be the particle's momentum. We want to express our equations in terms of $x$ and $p$. So $\dot{x}=p / m$ and $\dot{p}=m \ddot{x}=-V^{\prime}(x)$. So in first-order form our equations of motion become

$$
\begin{aligned}
\dot{x} & =\frac{p}{m} \\
\dot{p} & =-V^{\prime}(x) .
\end{aligned}
$$


Notice that (27) is a second-order equation on the line, whereas (28) is a firstorder equation on the plane. We have the following notation

$$
\begin{aligned}
\text { Line }(x \text {-space }) & =\text { configuration space } \\
\text { Plane }((x, p) \text {-space }) & =\text { phase space }
\end{aligned}
$$

In mechanics you must always be very clear about the distinction between the configuration space and the phase space.

We now consider the Hamiltonian form of mechanics. Let $H: \mathbb{R}^{2} \rightarrow \mathbb{R}$ be a smooth function (of the variables $x$ and $p$ ). Then define equations of motion on $\mathbb{R}^{2}$ by

$$
\begin{aligned}
\dot{x} & =\frac{\partial H}{\partial p} \\
\dot{p} & =-\frac{\partial H}{\partial x} .
\end{aligned}
$$

These are called Hamilton's equations. More explicitly these equations mean that we want trajectories $(x(t), p(t))$ satisfying

$$
\begin{aligned}
& \frac{d}{d t} x(t)=\frac{\partial H}{\partial p}(x(t), p(t)) \\
& \frac{d}{d t} p(t)=-\frac{\partial H}{\partial x}(x(t), p(t)) .
\end{aligned}
$$

The function $H$ is called the (classical) Hamiltonian function, and physically is the energy of the system.

Although $H$ can be any smooth function, it is often of the form

$$
H(x, p)=\frac{p^{2}}{2 m}+V(x),
$$

where the first term is the kinetic energy and the second is the potential energy. (Note that since $p=m \dot{x}, p^{2} / 2 m=\frac{1}{2} m(\dot{x})^{2}$, the usual expression for kinetic energy.) In that case, (29) becomes

$$
\begin{aligned}
& \dot{x}=\frac{\partial H}{\partial p}=\frac{p}{m} \\
& \dot{p}=-\frac{\partial H}{\partial x}=-V^{\prime}(x),
\end{aligned}
$$

which agrees with (28).

We need one more piece of fanciness to allow us to write classical mechanics in a way that allows a reasonable comparison with quantum mechanics.

Definition 22 If $f_{1}, f_{2}$ are smooth, real-valued function on $\mathbb{R}^{2}$, define the Poisson bracket of $f_{1}$ and $f_{2}$, denoted $\left\{f_{1}, f_{2}\right\}$, by

$$
\left\{f_{1}, f_{2}\right\}=\frac{\partial f_{1}}{\partial x} \frac{\partial f_{2}}{\partial p}-\frac{\partial f_{1}}{\partial p} \frac{\partial f_{2}}{\partial x}
$$

so that $\left\{f_{1}, f_{2}\right\}$ is another smooth function on $\mathbb{R}^{2}$. 
Theorem 23 If $f$ is any smooth function on $\mathbb{R}^{2}$ and $(x(t), p(t))$ is a solution to Hamilton's equations (29) then

$$
\frac{d}{d t} f(x(t), p(t))=\{f, H\}(x(t), p(t)),
$$

or more concisely,

$$
\frac{d f}{d t}=\{f, H\} .
$$

Proof. We use the chain rule, Hamilton's equations, and the definition of the Poisson bracket:

$$
\begin{aligned}
\frac{d f}{d t} & =\frac{\partial f}{\partial x} \frac{d x}{d t}+\frac{\partial f}{\partial p} \frac{d p}{d t} \\
& =\frac{\partial f}{\partial x} \frac{\partial H}{\partial p}-\frac{\partial f}{\partial p} \frac{\partial H}{\partial x} \\
& =\{f, H\} .
\end{aligned}
$$

Let us consider two examples. First let us take $f=H$ itself. Then

$$
\frac{d H}{d t}=\{H, H\}=\frac{\partial H}{\partial x} \frac{\partial H}{\partial p}-\frac{\partial H}{\partial p} \frac{\partial H}{\partial x}=0 .
$$

So energy is conserved! Next take $f$ to be the coordinate functions $x$ and $p$. Then we get

$$
\begin{aligned}
\frac{d x}{d t} & =\{x, H\}=\frac{\partial x}{\partial x} \frac{\partial H}{\partial p}-\frac{\partial x}{\partial p} \frac{\partial H}{\partial x} \\
& =\frac{\partial H}{\partial p}
\end{aligned}
$$

and

$$
\begin{aligned}
\frac{d p}{d t} & =\{p, H\}=\frac{\partial p}{\partial x} \frac{\partial H}{\partial p}-\frac{\partial p}{\partial p} \frac{\partial H}{\partial x} \\
& =-\frac{\partial H}{\partial x} .
\end{aligned}
$$

Thus Theorem 23 contains Hamilton's equation as a special case.

All of this can be done just as easily in $d$ dimensions. In that case the configuration space is $\mathbb{R}^{d}$ and the phase space is $\mathbb{R}^{2 d}$, with coordinates $x_{1}, \cdots, x_{d}, p_{1}, \cdots, p_{d}$. The Hamiltonian is then a smooth function $H(x, p)$ on $\mathbb{R}^{2 d}$, which is typically (but not always) of the form

$$
H(x, p)=\frac{1}{2 m} \sum_{k=1}^{d} p_{k}^{2}+V(x) .
$$


Hamilton's equations take the form

$$
\begin{aligned}
\frac{d x_{k}}{d t} & =\frac{\partial H}{\partial p_{k}} \\
\frac{d p_{k}}{d t} & =-\frac{\partial H}{\partial x_{k}} .
\end{aligned}
$$

The Poisson bracket is defined as

$$
\left\{f_{1}, f_{2}\right\}=\sum_{k=1}^{d} \frac{\partial f_{1}}{\partial x_{k}} \frac{\partial f_{2}}{\partial p_{k}}-\frac{\partial f_{1}}{\partial p_{k}} \frac{\partial f_{2}}{\partial x_{k}},
$$

and Theorem 23 holds:

$$
\frac{d f}{d t}=\{f, H\}
$$

\subsection{A very brief survey of quantum mechanics}

It takes some time and effort to understand what quantum mechanics is all about. Although I will try to explain enough to be comprehensible, you should not expect to understand the physical meaning of all this the first time through. I will describe the structure of quantum mechanics by analogy to the preceding

description of classical mechanics. But quantum mechanics is not supposed to be the same as classical mechanics; this is merely an analogy, not an equivalence.

Let us recall the structures that we had in classical mechanics.

- Phase space $\mathbb{R}^{2 d}$.

- Points $(x, p)$ in phase space.

- Real-valued functions $f$ on phase space.

- The value $f(x, p) \in \mathbb{R}$ of a function $f$ at a point $(x, p)$.

- The Poisson bracket $\left\{f_{1}, f_{2}\right\}$.

- The dynamics (equations of motion): $\frac{d f}{d t}=\{f, H\}$.

The fourth point may seem too obvious to be worth mentioning, but the quantum analog is not so obvious. Recall that the equation $d f / d t=\{f, H\}$ is implied by Hamilton's equations and contains them as a special case. I will now write down the corresponding structures in quantum mechanics.

- A complex Hilbert space $\mathcal{H}$.

- Unit vectors $\psi$ in $\mathcal{H}$, called "states."

- Self-adjoint linear operators $A$ on $\mathcal{H}$.

- The expected value of an operator $A$ in the state $\psi$, defined to be $\langle\psi, A \psi\rangle$. 
- The analog of the Poisson bracket for operators $A_{1}$ and $A_{2}$ :

$$
\frac{1}{i \hbar}\left[A_{1}, A_{2}\right]
$$

- The dynamics

$$
\frac{d A}{d t}=\frac{1}{i \hbar}[A, \hat{H}]
$$

Several points here require explanation. The most important point is that functions on the classical phase space correspond to operators on the quantum Hilbert space. We will discuss this correspondence in detail later. Note also that there is a parameter $\hbar$ in the quantum theory, which is Planck's constant and which has no classical analog. We will regard $\hbar$ as merely a parameter, although physically it is an experimentally determined constant, whose numerical value depends on the system of units. (It is small compared to the scale of everyday life.) The process of converting from the classical picture to the quantum picture is called "quantization." There is in general no hard-and-fast rule for how to quantize things, although there are well-established procedures in certain important cases.

The dynamics in the quantum picture requires a whole discussion itself. The quantity $\hat{H}$ is an operator on the quantum Hilbert space, called the (quantum) Hamiltonian operator. By analogy with the classical picture we assume that there is some distinguished self-adjoint operator $\hat{H}$ (determined by theoretical or experimental considerations) which governs the dynamics. Then the dynamics of the states $\psi \in \mathcal{H}$ is assumed to satisfy the Schrödinger equation

$$
i \hbar \frac{d \psi}{d t}=\hat{H} \psi \text {. }
$$

We can then use this to determine how the expectation value of some operator $A$ varies in time in a state $\psi(t)$ satisfying the Schrödinger equation. We compute

$$
\begin{aligned}
\frac{d}{d t}\langle\psi(t), A \psi(t)\rangle & =\left\langle\frac{d \psi}{d t}, A \psi\right\rangle+\left\langle\psi, A \frac{d \psi}{d t}\right\rangle \\
& =\left\langle\frac{1}{i \hbar} \hat{H} \psi, A \psi\right\rangle+\left\langle\psi, A \frac{1}{i \hbar} \hat{H} \psi\right\rangle \\
& =-\frac{1}{i \hbar}\langle\psi, \hat{H} A \psi\rangle+\frac{1}{i \hbar}\langle\psi, A \hat{H} \psi\rangle \\
& =\frac{1}{i \hbar}\langle\psi,[A, \hat{H}] \psi\rangle .
\end{aligned}
$$

Between the second and third lines I have used the fact that $\hat{H}$ is self-adjoint. So

$$
\frac{d}{d t}\langle\psi(t), A \psi(t)\rangle=\left\langle\psi(t), \frac{1}{i \hbar}[A, \hat{H}] \psi(t)\right\rangle
$$


or (suppressing the dependence on the state as in the classical theory)

$$
\frac{d A}{d t}=\frac{1}{i \hbar}[A, \hat{H}] .
$$

This is the Heisenberg form of the Schrödinger equation. This form emphasizes the analogy with classical mechanics.

Example: $\mathcal{H}=L^{2}\left(\mathbb{R}^{d}, d x\right)$.

In the "standard" quantization scheme the quantum Hilbert space is $L^{2}\left(\mathbb{R}^{d}, d x\right)$. Note that this is $L^{2}$ of configuration space, not $L^{2}$ of phase space! The classical function $x_{k}$ corresponds the operator $X_{k}$ (multiplication by $x_{k}$ ) and the classical function $p_{k}$ corresponds to the operator $P_{k}=-i \hbar \partial / \partial x_{k}$. A classical function of the form $H(x, p)=p^{2} / 2 m+V(x)$ corresponds to the operator

$$
\begin{aligned}
\hat{H} & =\frac{P^{2}}{2 m}+V(X) \\
& =-\frac{\hbar^{2}}{2 m} \Delta+V(X) .
\end{aligned}
$$

Here $V(X)$ means multiplication by $V(x)$ and $P^{2}=P_{1}^{2}+\cdots+P_{k}^{2}$.

Let us compare the classical Poisson bracket to the commutator of the corresponding operators.

$$
\begin{aligned}
\left\{x_{k}, p_{l}\right\} & =\sum_{j} \frac{\partial x_{k}}{\partial x_{j}} \frac{\partial p_{l}}{\partial p_{j}}-\frac{\partial x_{k}}{\partial p_{j}} \frac{\partial p_{l}}{\partial x_{j}} \\
& =\delta_{k, l}
\end{aligned}
$$

and

$$
\begin{aligned}
\frac{1}{i \hbar}\left[X_{k}, P_{l}\right] & =\frac{1}{i \hbar} i \hbar \delta_{k, l} I \\
& =\delta_{k, l} I .
\end{aligned}
$$

A comparison of (36) and (37) explains in part the canonical commutation relations.

Let's try another example.

$$
\begin{aligned}
\left\{x_{k}, H\right\} & =\sum_{j} \frac{\partial x_{k}}{\partial x_{j}} \frac{\partial H}{\partial p_{j}}-\frac{\partial x_{k}}{\partial p_{j}} \frac{\partial H}{\partial x_{j}} \\
& =2 p_{k} .
\end{aligned}
$$

And

$$
\begin{aligned}
\frac{1}{i \hbar}\left[X_{k}, \hat{H}\right] & =\frac{1}{i \hbar}\left[X_{k}, \frac{1}{2 m} P^{2}+V\right] \\
& =\frac{1}{i \hbar} \frac{1}{2 m}\left[X_{k}, P_{k}^{2}\right],
\end{aligned}
$$


since multiplication by $x_{k}$ commutes with multiplication by $V(x)$ and with $\partial / \partial x_{l}(k \neq l)$. Using Point (d) of Exercise $24\left[X_{k}, P_{k}^{2}\right]=\left[X_{k}, P_{k}\right] P_{k}+$ $P_{k}\left[X_{k}, P_{k}\right]=2 i \hbar P_{k}$. So

$$
\frac{1}{i \hbar}\left[X_{k}, \hat{H}\right]=2 P_{k}
$$

One more encouraging example before things start to get problematical.

$$
\begin{aligned}
\left\{p_{k}, H\right\} & =\sum_{j} \frac{\partial p_{k}}{\partial x_{j}} \frac{\partial H}{\partial p_{j}}-\frac{\partial p_{k}}{\partial p_{j}} \frac{\partial H}{\partial x_{j}} \\
& =-\frac{\partial V(x)}{\partial x_{k}} .
\end{aligned}
$$

and

$$
\begin{aligned}
\frac{1}{i \hbar}\left[P_{k}, \hat{H}\right] & =\frac{1}{i \hbar}\left[P_{k}, V(X)\right] \\
& =-\frac{\partial}{\partial x_{k}} V(x)+V(x) \frac{\partial}{\partial x_{k}} \\
& =-\frac{\partial V(x)}{\partial x_{k}} .
\end{aligned}
$$

In the three previous examples the classical Poisson bracket seems to correspond exactly with the quantum commutator. (Compare (36) and (37), (38) and (39), and (40) and (41).) But things are not always so simple. Skipping the algebra I will record that (in $d=1$ )

$$
\begin{aligned}
\left\{x^{3}, p^{2}\right\} & =6 x^{2} p \\
\frac{1}{i \hbar}\left[X^{3}, P^{2}\right] & =3\left(X^{2} P+P X^{2}\right) .
\end{aligned}
$$

Is $3\left(X^{2} P+P X^{2}\right)$ the operator which corresponds to the classical function $6 x^{2} p$ ? It's not so clear is it? Although classically $x^{2} p=p x^{2}=x p x$, on the quantum side $X^{2} P, P X^{2}$, and $X P X$ are all different, so it is not evident what the quantum operator should be. We need some sort of systematic theory here, which will come in the next subsection.

\subsection{Quantization schemes}

Dirac, one of the founders of quantum mechanics, proposed the following axiomatic approach to quantization. Dirac wanted a Hilbert space $\mathcal{H}$ and a map

$$
Q \text { : functions on the phase space } \mathbb{R}^{2 d} \rightarrow \text { operators on } \mathcal{H}
$$

with the following properties.

1. $Q$ is linear and $Q(1)=I$. 
2. $Q(\{f, g\})=\frac{1}{i \hbar}[Q(f), Q(g)]$.

3. $\mathcal{H}$ is irreducible under the action of $Q\left(x_{k}\right)$ and $Q\left(p_{k}\right)$

$3^{\prime} \mathcal{H}=L^{2}\left(\mathbb{R}^{d}, d x\right), Q\left(x_{k}\right)=$ multiplication by $x_{k}$, and $Q\left(p_{k}\right)=-i \hbar \partial / \partial x_{k}$.

4. If $f$ is real-valued, then $Q(f)$ is self-adjoint.

Note that the canonical commutation relations are implied by (1) and (2), in light of the fact that $\left\{x_{k}, p_{l}\right\}=\delta_{k, l}$. Note also that (3) and $\left(3^{\prime}\right)$ are more or less equivalent in the presence of (1) and (2), by the Stone-von Neumann Theorem. Also, the map $Q$ clearly depends on $\hbar$ (in light of (2)), but I am suppressing this dependence in my notation. This is a reasonable axiomatic system, except for the following result.

Theorem 24 (Groenewold-van Hove Theorem) There is no map $Q$ satisfying (1), (2), (3), and (4).

I should emphasize that I am being very seriously imprecise in the statement of this theorem. The real theorem has precise domain conditions, and they are necessary to get a real theorem. See Theorem 4.59 and the following discussion in $\mathrm{F}$, or $\mathrm{Gg}]$.

The "prequantization" map of geometric quantization [W] satisfies (1), (2), and (4), but not (3). However, this is not supposed to be quantization, but only prequantization. That is, condition (3) is important and cannot simply be abandoned.

So we have a bit of problem, and the sad fact of the matter is that there is no neat mathematical principle that tells us how to proceed. Ultimately the test of whether we have the right "quantization scheme" comes from experiment. But it is generally accepted that we should keep (1) and (3), and require (2) to hold at least for the coordinate functions $x_{k}$ and $p_{k}$. Thus we require that the canonical commutation relations hold! This explains the importance of the canonical commutation relations from the point of view of quantum mechanics.

The Stone-von Neumann Theorem says that if you accept (1) and (3) and the CCRs (a special case of (2)), then up to unitary equivalence we may as well take $\left(3^{\prime}\right)$ as well. This then determines what our Hilbert space should be and what $Q\left(x_{k}\right)$ and $Q\left(p_{k}\right)$ should be. But then we have nothing that tells us what, say, $Q\left(x_{k} p_{k}\right)$ should be. There are several different ways of defining $Q$ assuming $(1)$ and $\left(3^{\prime}\right)$. I will describe these in the case $d=1$, though all can be extended to arbitrary $d$.

(a) Put all the $X$ 's to the left and all the P's to the right.

This is called the (standard) pseudodifferential operator quantization. So this map should send the function $x^{m} p^{n}$ to $X^{n} P^{m}$. For general functions $f(x, p)$ this quantization can be described in terms of the Fourier transform as follows.

$$
Q(f) \psi(x)=\frac{1}{2 \pi} \int_{\mathbb{R}} f(x, \hbar \xi) \hat{\psi}(\xi) d \xi,
$$


where $\psi \in L^{2}(\mathbb{R}, d x)$ and $\hat{\psi}(\xi)=\int e^{-i \xi x} \psi(x) d x$ is the Fourier transform of $\psi$. There is a large theory relating properties of $f$ to properties of $Q(f)$ in this quantization scheme. However, the pseudo-differential quantization does not satisfy property (4) and so is not a good candidate for the physical quantization map. (Consider just the example $f(x, p)=x p$.) It is nevertheless an important map in the theory of differential equations.

(b) Put all the $X$ 's to the right and all the $P$ 's to the left.

This is similar of course to (a).

(c) Symmetric or Weyl ordering.

This scheme and the two remaining ones satisfy property (4).

The Weyl quantization is probably the best candidate physically for the right quantization scheme for general functions. It has the property that

$$
Q\left(x^{n} p^{m}\right)=\frac{1}{(n+m) !} \sum_{\sigma \in S_{n+m}} \sigma \cdot\left(X^{n} P^{m}\right) .
$$

Here $S_{n+m}$ is the permutation group on $n+m$ objects, and $\sigma \cdot\left(X^{n} P^{m}\right)$ is schematic notation for what you get by permuting the $n+m$ factors in $X^{n} P^{m}$. So for example if $n=2, m=1$, then we have permutations of 3 objects, listable as $(1,2,3),(1,3,2),(2,1,3),(2,3,1),(3,1,2),(3,2,1)$. So applying these permutations to $X X P$ gives

$$
\begin{aligned}
Q\left(x^{2} p\right) & =\frac{1}{6}[X X P+X P X+X X P+X P X+P X X+P X X] \\
& =\frac{1}{3}\left[X^{2} P+X P X+P X^{2}\right]
\end{aligned}
$$

Of course, many of the terms you get will be the same; actually you need just one instance of each distinct ordering of $n X$ 's and $m$ P's, but I couldn't think of any compact way of writing this.

The Weyl quantization is characterized by the fact that

$$
Q\left(e^{i a x+i b p}\right)=e^{i a X+i b P} .
$$

Thus for general $f$ we have

$$
Q(f)=\left(\frac{1}{2 \pi}\right)^{2} \int_{\mathbb{R}^{2}} e^{i a X+i b P} \hat{f}(a, b) d a d b .
$$

It is not obvious but true that (44) implies (43). See [F] Chapter 2.1, especially Eq. (2.20) and the following paragraph.

(d) The Wick ordering or normal ordering.

This quantization scheme is very important in quantum field theory. Recall the annihilation and creation operators

$$
\begin{aligned}
a & =\frac{X+i P}{\sqrt{2}} \\
a^{*} & =\frac{X-i P}{\sqrt{2}} .
\end{aligned}
$$


Wick ordering puts the annihilation operators to the right (acting first) and the creation operators to the left. That is,

$$
\begin{aligned}
Q\left((x-i p)^{n}(x+i p)^{m}\right) & =(X-i P)^{n}(X+i P)^{m} \\
& =2^{(m+n) / 2}\left(a^{*}\right)^{m} a^{n} .
\end{aligned}
$$

As an example let's consider the function $\frac{1}{2}\left(x^{2}+p^{2}\right)=\frac{1}{2}(x-i p)(x+i p)$. This quantizes to

$$
\begin{aligned}
\frac{1}{2}(X-i P)(X+i P) & =\frac{1}{2}\left(X^{2}+P^{2}+i(X P-P X)\right) \\
& =\frac{1}{2}\left(X^{2}+P^{2}+i(i \hbar)\right) \\
& =\frac{1}{2}\left(X^{2}+P^{2}\right)-\frac{\hbar}{2} .
\end{aligned}
$$

I will leave it as an exercise to calculate that in this scheme

$$
Q\left(x^{2}\right)=X^{2}-\frac{\hbar}{2} .
$$

So in contrast to (a), (b), and (c), in this quantization scheme $Q\left(x^{n}\right) \neq Q(x)^{n}$.

(e) The anti-Wick or anti-normal ordering.

This is the reverse of Wick ordering, namely,

$$
\begin{aligned}
Q\left((x-i p)^{n}(x+i p)^{m}\right) & =(X+i P)^{m}(X-i P)^{n} \\
& =2^{(m+n) / 2} a^{n}\left(a^{*}\right)^{m} .
\end{aligned}
$$

So in this ordering the creation operators go to the right (acting first). Imitating the above computation shows that in this ordering

$$
\begin{aligned}
Q\left(\frac{1}{2}\left(x^{2}+p^{2}\right)\right) & =\frac{1}{2}\left(X^{2}+P^{2}\right)+\frac{\hbar}{2} \\
Q\left(x^{2}\right) & =X^{2}+\frac{\hbar}{2} .
\end{aligned}
$$

The anti-Wick ordering can be described very naturally in terms of Toeplitz operators, as we shall see in Section 8. The anti-Wick ordering also has nice properties that none of the other orderings have.

\subsection{The significance of the Segal-Bargmann representa- tion}

It is worthwhile at this point to make some remarks about the significance of the Segal-Bargmann space and the associated transform. Although the SegalBargmann space is naturally connected to the anti-Wick ordering (as we shall see in Section 8), it is also useful with other quantization schemes, for example the Weyl ordering. I think of the Segal-Bargmann space as simply a different 
but unitarily equivalent representation of the canonical commutation relations. So it is not so much a different quantization as a unitarily equivalent realization of the same quantization. The value of the Segal-Bargmann transform lies in the fact that this unitary transformation makes certain problems easier to work with. Certainly for semiclassical analysis (in which one tries to relate quantum theory to classical theory) it is very natural to use the Segal-Bargmann representation, because it is a Hilbert space of functions on the phase space $\mathbb{R}^{2 d}=\mathbb{C}^{d}$ rather than on the configuration space $\mathbb{R}^{d}$. Since classical mechanics is naturally formulated in phase space, this is a big advantage. In WKB theory (which is an important part of semiclassical analysis), the Segal-Bargmann representation also has technical advantages over the traditional $L^{2}\left(\mathbb{R}^{d}\right)$ representation. (See Voros [V], Paul-Uribe |PU,, Graffi-Paul [GP], Thomas-Wassell [TW], and Borthwick-Paul-Uribe [BPU], [Bo].) The Segal-Bargmann space is also the natural home for the Husimi function, which is gaining popularity in physics and which we will describe in the next section, and for the Wick and anti-Wick quantization schemes.

\subsection{Exercises}

Exercise 23 Show that the Poisson bracket on $\mathbb{R}^{2 d}$ has the following properties:

a) (Skew-symmetry) $\left\{f_{1}, f_{2}\right\}=-\left\{f_{2}, f_{1}\right\}$.

b) (Bilinearity) $\left\{f_{1}, f_{2}\right\}$ is linear with respect to $f_{1}$ with $f_{2}$ fixed, and vice versa.

c) (Jacobi identity) $\left\{f_{1},\left\{f_{2}, f_{3}\right\}\right\}+\left\{f_{2},\left\{f_{3}, f_{1}\right\}\right\}+\left\{f_{3},\left\{f_{1}, f_{2}\right\}\right\}=0$.

d) $\left\{f_{1}, f_{2} f_{3}\right\}=\left\{f_{1}, f_{2}\right\} f_{3}+f_{2}\left\{f_{1}, f_{3}\right\}$.

Point (d) says that $\left\{f_{1}, \cdot\right\}$ is a derivation; that it, it is a Leibniz-type product rule for the Poisson bracket.

Exercise 24 Show that the commutator of operators $[A, B]:=A B-B A$ has the following properties:

a) (Skew-symmetry) $[A, B]=-[B, A]$.

b) (Bilinearity) $[A, B]$ is linear with respect to $A$ with $B$ fixed and vice versa.

c) (Jacobi identity) $[A,[B, C]]+[B,[C, A]]+[C,[A, B]]=0$.

d) $[A, B C]=[A, B] C+B[A, C]$.

Exercise 25 Verify that the pseudodifferential quantization, as given by (4A), satisfies $Q\left(x^{n} p^{m}\right)=X^{n} P^{m}$.

\section{Toeplitz operators, anti-Wick ordering, and phase space probability densities}

The anti-Wick ordering can be expressed in an analytically nice way in terms of Toeplitz operators on the Segal-Bargmann space. This will lead us to the notion of phase space probability densities, one of which (the Husimi function) also is most naturally expressed in terms of the Segal-Bargmann space. 


\subsection{General theory of Toeplitz operators}

Let us return to the general setting of holomorphic function spaces, $\mathcal{H} L^{2}(U, \alpha)$. Recall that $\mathcal{H} L^{2}(U, \alpha)$ is a closed subspace of $L^{2}(U, \alpha)$, and therefore there is an orthogonal projection operator $P: L^{2}(U, \alpha) \rightarrow \mathcal{H} L^{2}(U, \alpha)$. We showed that this projection is given in terms of the reproducing kernel as

$$
P F(z)=\int_{U} K(z, w) F(w) \alpha(w) d w
$$

for all $F \in L^{2}(U, \alpha)$.

Now suppose that $\phi$ is any bounded measurable function on $U$. Define a linear operator

$$
T_{\phi}: \mathcal{H} L^{2}(U, \alpha) \rightarrow \mathcal{H} L^{2}(U, \alpha)
$$

by

$$
T_{\phi} F=P(\phi F), \quad F \in \mathcal{H} L^{2}(U, \alpha) .
$$

This is called the Toeplitz operator with symbol $\phi$. So a Toeplitz operator is one of the form "multiply then project," that is, multiply by $\phi$ and then project back into the holomorphic subspace.

Theorem 25 Toeplitz operators have the following properties.

1. $\left\|T_{\phi}\right\| \leq\|\phi\|_{L^{\infty}}$.

2. $T_{\phi}$ is linear as a function of $\phi$.

3. $T_{1}=I$.

4. $T_{\bar{\phi}}=\left(T_{\phi}\right)^{*}$. In particular, if $\phi$ is real then $T_{\phi}$ is self-adjoint.

5. For all $F_{1}, F_{2} \in \mathcal{H} L^{2}(U, \alpha)$,

$$
\left\langle F_{1}, T_{\phi} F_{2}\right\rangle=\left\langle F_{1}, \phi F_{2}\right\rangle .
$$

Proof. We can think of $T_{\phi}$ as an operator on all of $L^{2}(U, \alpha)$ by setting it to zero on the orthogonal complement of the holomorphic subspace. In that case we can write

$$
T_{\phi}=P M_{\phi} P
$$

where $M_{\phi}$ denotes multiplication by $\phi$. Thus

$$
\begin{aligned}
\left\|T_{\phi}\right\| & \leq\|P\|\left\|M_{\phi}\right\|\|P\| \\
& =\|\phi\|_{L^{\infty}}
\end{aligned}
$$


and

$$
\begin{aligned}
\left(T_{\phi}\right)^{*} & =P^{*}\left(M_{\phi}\right)^{*} P^{*} \\
& =P M_{\bar{\phi}} P=T_{\bar{\phi}} .
\end{aligned}
$$

This establishes Point 1 and Point 4. Points 2 and 3 are clear.

For Point 5 we observe that

$$
\begin{aligned}
\left\langle F_{1}, T_{\phi} F_{2}\right\rangle & =\left\langle F_{1}, P \phi F_{2}\right\rangle \\
& =\left\langle P F_{1}, \phi F_{2}\right\rangle \\
& =\left\langle F_{1}, \phi F_{2}\right\rangle
\end{aligned}
$$

since $P$ is self-adjoint and $F_{1}$ is assumed to be holomorphic.

Now if $\phi$ is an unbounded function, then we can still define the Toeplitz operator $T_{\phi}$ in the same way, except that $T_{\phi}$ may be unbounded. I will assume that $\phi$ is nice enough that $T_{\phi}$ is at least densely defined. We expect that Point 4 of the theorem still holds modulo domain issues (which we are not worrying about in these notes). The following result is asserted and proved without worrying about domain issues.

Theorem 26 Suppose $\phi_{1}, \cdots, \phi_{n}$ and $\psi_{1}, \cdots, \psi_{m}$ are holomorphic. Then

$$
T_{\bar{\psi}_{1} \cdots \bar{\psi}_{m} \phi_{1} \cdots \phi_{n}}=T_{\bar{\psi}_{1}} \cdots T_{\bar{\psi}_{m}} T_{\phi_{1}} \cdots T_{\phi_{n}} .
$$

Proof. If $\phi_{1}, \cdots, \phi_{n}$ are holomorphic then

$$
T_{\phi_{1}} \cdots T_{\phi_{n}}=P M_{\phi_{1}} P P M_{\phi_{2}} P \cdots P M_{\phi_{n}} P .
$$

But all the projections except the first are unnecessary, since the $\phi$ 's are holomorphic. So

$$
\begin{aligned}
T_{\phi_{1}} \cdots T_{\phi_{n}} & =P M_{\phi_{1}} \cdots M_{\phi_{n}} P \\
& =T_{\phi_{1} \cdots \phi_{n}} .
\end{aligned}
$$

Taking the adjoint of this we see that

$$
T_{\bar{\psi}_{1} \cdots \bar{\psi}_{m}}=T_{\bar{\psi}_{1}} \cdots T_{\bar{\psi}_{m}} .
$$

Finally,

$$
\begin{aligned}
T_{\bar{\psi}_{1} \cdots \bar{\psi}_{m} \phi_{1} \cdots \phi_{n}} & =P M_{\bar{\psi}_{1} \cdots \bar{\psi}_{m}} M_{\phi_{1} \cdots \phi_{n}} P \\
& =P M_{\bar{\psi}_{1} \cdots \bar{\psi}_{m}} P M_{\phi_{1} \cdots \phi_{n}} P \\
& =T_{\bar{\psi}_{1} \cdots \bar{\psi}_{m}} T_{\phi_{1} \cdots \phi_{n}} \\
& =T_{\bar{\psi}_{1}} \cdots T_{\bar{\psi}_{m}} T_{\phi_{1}} \cdots T_{\phi_{n}}
\end{aligned}
$$




\subsection{Toeplitz operators on the Segal-Bargmann space}

If we consider Toeplitz operators in the Segal-Bargmann space, $\mathcal{H} L^{2}\left(\mathbb{C}, \mu_{\hbar}\right)$, then

$$
T_{z}=z
$$

(the projection being unnecessary since $z$ is holomorphic) and

$$
T_{\bar{z}}=\left(T_{z}\right)^{*}=\hbar \frac{d}{d z} .
$$

These are the creation and annihilation operators. So Theorem 26 says that

$$
T_{\bar{z}^{n} z^{m}}=\left(\hbar \frac{d}{d z}\right)^{n} z^{m}
$$

Note that the $z^{m}$ 's, which are the creation operators, are to the right and the $(\hbar d / d z)$ 's, which are the annihilation operators, are to the left. So this is clearly closely related to the anti-Wick ordering. But there are some pesky minus signs and $\sqrt{2}$ 's to be dealt with. The creation operator, recall, is the operator

$$
\frac{X-i P}{\sqrt{2}}=a^{*} \Rightarrow T_{z}
$$

whereas the annihilation operator is

$$
\frac{X+i P}{\sqrt{2}}=a \Rightarrow T_{\bar{z}}
$$

where $\Rightarrow$ means "corresponds to under the Segal-Bargmann transform." So $x+i p$ does not correspond to $T_{z}$. To fix this we need a minus sign and a $\sqrt{2}$.

Theorem 27 Given a function $\phi$ on $\mathbb{C}^{d}$, define another function $\phi^{\prime}$ on $\mathbb{C}^{d}$ by

$$
\phi^{\prime}(z)=\phi(\sqrt{2} \bar{z})
$$

For each $\phi$, consider the Toeplitz operator $T_{\phi^{\prime}}$ as an operator on the SegalBargmann space $\mathcal{H} L^{2}\left(\mathbb{C}^{d}, \mu_{\hbar}\right)$. Then the map $\phi \rightarrow T_{\phi^{\prime}}$ is unitarily equivalent to the anti-Wick ordering. More precisely, for any $\phi$, the operator $A_{\hbar}^{-1} T_{\phi^{\prime}} A_{\hbar}$ on $L^{2}\left(\mathbb{R}^{d}\right)$ is the same as the anti-Wick quantization of $\phi$.

If we use the "invariant" form of the Segal-Bargmann space, $\mathcal{H} L^{2}\left(\mathbb{C}^{d}, \nu_{\hbar}\right)$, then we get a similar theorem without the factor of $\sqrt{2}$. But even then it is necessary to include the complex conjugate. If one is willing to use anti-holomorphic functions (as in Segal) instead of holomorphic functions, then one can get rid of the complex conjugate as well. 


\subsection{Wigner function and Husimi function}

In the conventional quantization, in which the Hilbert space is $L^{2}\left(\mathbb{R}^{d}, d x\right)$, if $\psi$ is a unit vector, then we interpret $|\psi(x)|^{2}$ as the "position probability density." This means that in quantum theory the particle does not have a definite position, but only a probability distribution for the position, whose density is given by $|\psi(x)|^{2}$. By using a Fourier transform, one can also associate to each unit vector $\psi$ a "momentum probability density." A natural next step is to ask, can you define something like a joint distribution of position and momentum, which we would call a "phase space probability density"? A little exposure to quantum mechanics will convince you that there is no notion of a phase space probability density that has all the properties you would like. (Certainly taking just the product of the position and momentum distributions is much too simplistic.) Still, we can still try for something that has enough reasonable properties to be useful.

On reasonable way to try to define a phase space probability density is to pick a Hilbert space $\mathcal{H}$ and a quantization scheme $Q$. Then for each unit vector $\psi \in \mathcal{H}$ we can look for a probability density $p_{\psi}$ on the phase space $\mathbb{R}^{2 d}$ satisfying

$$
\int_{\mathbb{R}^{2 d}} f(x, p) p_{\psi}(x, p) d x d p=\langle\psi, Q(f) \psi\rangle_{\mathcal{H}}
$$

for all nice functions $f$. This condition is reasonable because in probabilistic language the left side is the expectation value of the function $f$ with respect to the probability density $p_{\psi}$. Meanwhile the right side is what we have called the expectation value of the corresponding operator $Q(f)$ in the state $\psi$.

Note that (47) serves to define $p_{\psi}$ as a distribution (or generalized function), provided only that $\psi$ is in the domain of $Q(f)$ whenever $f$ is a $\mathcal{C}^{\infty}$ function of compact support. (For most quantization schemes, $Q(f)$ is bounded whenever $f$ is $\mathcal{C}^{\infty}$ and of compact support.) Furthermore, taking $f \equiv 1$ we see that the total integral of $p_{\psi}$ is one, since $Q(1)=I$ and $\psi$ is a unit vector. Unfortunately, $p_{\psi}$ will not be positive in general.

The first example of interest is the Weyl quantization, in which case the associated $p_{\psi}$ is called the Wigner function. This is the most natural thing to try, since the Weyl quantization is the most physically natural quantization scheme. Unfortunately, the Wigner function is not always positive. That is, for most unit vectors $\psi \in L^{2}\left(\mathbb{R}^{d}, d x\right), p_{\psi}$ is negative at certain points. In fact, the only functions $\psi$ for which $p_{\psi}$ is everywhere non-negative are ones of the form $\psi(x)=$ const. $\exp \left(-(x-a)^{2} / b\right) \exp (i x \cdot c)$, with $a, c \in \mathbb{R}^{d}$ and $b \in(0, \infty)$. The Wigner function is therefore in general called a pseudo-probability density.

The other main example of interest is the anti-Wick quantization, in which case the associated $p_{\psi}$ is called the Husimi function. Although this is not as natural sounding as the Wigner function because the anti-Wick quantization is not as natural, the Husimi function has the very nice property that it is always positive (that is, non-negative). We see this explicitly in the following theorem.

Theorem 28 For $\psi \in L^{2}\left(\mathbb{R}^{d}, d x\right)$ with $\|\psi\|=1$, the Husimi function of $\psi$, 
denoted $H_{\psi}$, is given by

$$
H_{\psi}(x, p)=\left|A_{\hbar} \psi\right|^{2}\left(\frac{x-i p}{\sqrt{2}}\right) \frac{e^{-|z|^{2} / 2 \hbar}}{(2 \pi \hbar)^{d}} .
$$

where $A_{\hbar}$ is the Segal-Bargmann transform.

In terms of the invariant form of the Segal-Bargmann transform we have

$$
H_{\psi}(x, p)=\left|C_{\hbar} \psi\right|^{2}(x-i p) \frac{e^{-(\operatorname{Im} z)^{2} / \hbar}}{(\pi \hbar)^{-d / 2}} .
$$

This result follows almost immediately from Theorem 27 and the analogous result for $C_{\hbar}$. The reader may verify using (26) from Section 6 that the two expressions for $H_{\psi}$ are indeed equal.

The following theorem describes the relationship between the anti-Wick and Weyl quantizations, and correspondingly between the Husimi function and the Wigner function.

Theorem 29 1. For all bounded measurable functions $f$,

$$
Q_{\text {anti-Wick }}(f)=Q_{W e y l}\left(e^{\hbar \Delta / 4} f\right)
$$

where $\Delta$ is the standard Laplacian on $\mathbb{R}^{2 d}$ and $e^{\hbar \Delta / 4} f$ is given explicitly by

$$
e^{\hbar \Delta / 4} f(z)=(\pi \hbar)^{-d} \int_{\mathbb{C}^{d}} e^{-|z-u|^{2} / \hbar} f(u) d u .
$$

2. For all $\psi \in L^{2}\left(\mathbb{R}^{d}, d x\right)$ with norm one,

$$
H_{\psi}(z)=(\pi \hbar)^{-d} \int_{\mathbb{C}^{d}} e^{-|z-u|^{2} / \hbar} W_{\psi}(u) d u,
$$

where $H_{\psi}$ is the Husimi function and $W_{\psi}$ is the Wigner function.

Point 2 of the theorem is often described by saying that the Husimi function is obtained by "smearing out" the Wigner function, by convolving it with a Gaussian (whose "width" is proportional to $\sqrt{\hbar}$.) It is interesting that this smearing is just enough to make the Husimi function always positive, even when the Wigner function is not. A proof of Point 1 is found in [F]; Point 2 then follows.

Let us compare further the Husimi and Wigner functions. Once nice property of the Wigner function is that it properly reproduces the "marginal distributions" of $x$ and $p$. That is, if you take $W_{\psi}(x, p)$ and integrate out the $p$-dependence, you obtain the standard position probability density, and similarly with $x$ and $p$ reversed. That is, we have the following result. 
Theorem 30 For $\psi \in L^{2}\left(\mathbb{R}^{d}, d x\right)$ with $\|\psi\|=1$, the Wigner function $W_{\psi}$ satisfies

$$
\int_{\mathbb{R}^{d}} W_{\psi}(x, p) d p=|\psi(x)|^{2}
$$

and

$$
\int_{\mathbb{R}^{d}} W_{\psi}(x, p) d x=|\tilde{\psi}(p)|^{2}
$$

where $\tilde{\psi}$ is the $\hbar$-scaled Fourier transform:

$$
\tilde{\psi}(p)=(2 \pi \hbar)^{-d / 2} \int_{\mathbb{R}^{d}} e^{i p \cdot x / \hbar} \psi(x) d x .
$$

Note that the marginal distributions of $W_{\psi}$ are positive even if $W_{\psi}$ is not positive. The reason for this result is that the Weyl quantization has the property that $Q\left(x^{n}\right)=X^{n}$ and $Q\left(p^{n}\right)=P^{n}$ (and more generally $Q(f(x))=f(X)$ and $Q(f(p))=f(P))$. The anti-Wick quantization does not have this property, so it does not have the desired marginal distributions. But there is a compensating benefit, namely a result that gives the position and momentum wave functions in terms of the "phase space wave function," namely, the SegalBargmann transform, where the Husimi function is essentially just the absolute value squared of the Segal-Bargmann transform. It is easiest to state this in terms of the invariant form $C_{\hbar}$ of the Segal-Bargmann transform.

Theorem 31 If $\psi \in L^{2}\left(\mathbb{R}^{d}, d x\right)$ then

$$
\psi(x)=(2 \pi \hbar)^{-d / 2} \int_{\mathbb{R}^{d}} C_{\hbar} \psi(x+i p) e^{-p^{2} / 2 \hbar} d p
$$

and

$$
\tilde{\psi}(p)=(2 \pi \hbar)^{-d / 2} e^{-p^{2} / 2 \hbar} \int_{\mathbb{R}^{d}} C_{\hbar} \psi(x+i p) d p
$$

where $\tilde{\psi}$ is as in the previous theorem.

In both theorems I am glossing over convergence issues. If you assume that $\psi$ is nice enough then all formulas can be taken literally. But for general $\psi \in L^{2}, \psi$ can diverge at certain points and there must correspondingly be some divergences in the integrals. In the second theorem, for example, one can deal with this by integrating over a ball of radius $R$ and then taking an $L^{2}$ limit as $R \rightarrow \infty$.

\subsection{Exercises}

Exercise 26 Verify Point 1 of Theorem 29 in the case $f(x, p)=\frac{1}{2}\left(x^{2}+p^{2}\right)$. Hint: if $f$ is a polynomial, then $e^{\hbar \Delta / 4}$ can be computed by expanding it in a power series. 
Exercise $27{ }^{*}$ Verify Point 1 of Theorem 29 if $f(x, p)=x^{n}$.

Exercise 28 If $\phi$ is a positive function, show that the Toeplitz operator $T_{\phi}$ is a positive operator. Use this to explain why the Husimi function is always positive.

Exercise 29 Show that for all unit vectors $\psi$, the Husimi function $H_{\psi}$ satisfies

$$
H_{\psi}(z) \leq(2 \pi \hbar)^{-d}
$$

for all $z \in \mathbb{C}^{d}$. This is a form of the uncertainty principle, namely a limit on how concentrated a state can be in phase space. (After all, $H_{\psi}$ integrates to one. So if it can't be too big at any one point, it must be fairly spread out.)

Exercise 30 The functions $\psi_{z}$ which give equality in the previous problem for a given value of $z$ are called the coherent states. Compute the Husimi function of the coherent states.

\section{The Segal-Bargmann transform for compact Lie groups}

\subsection{Beyond the Canonical Commutation Relations}

I have introduced the ordinary Segal-Bargmann transform from the point of view of the canonical commutation relations, a point of view that fits well with the way Segal and Bargmann described the transform. I now want to describe a generalization of the Segal-Bargmann transform in which the configuration space $\mathbb{R}^{d}$ is replaced by a compact Lie group. In this generalized setting there are no canonical commutation relations. So having described the ordinary SegalBargmann transform entirely in terms of the CCRs, I am now going to describe a generalization of the Segal-Bargmann transform that does not involve CCRs at all! Although this may seem strange, there is a good reason for abandoning the CCRs, namely that in a more general setting there seems to be no good candidate for what the CCRs ought to be. Recall that in the $\mathbb{R}^{d}$ case, the CCRs are the quantum-mechanical analog of the Poisson bracket relations $\left\{x_{k}, p_{l}\right\}=$ $\delta_{k, l}$. We are now going to replace the configuration space $\mathbb{R}^{d}$ by a compact Lie group $K$. (This will be explained below.) Correspondingly we replace the phase space $\mathbb{R}^{2 d}$ by the cotangent bundle of $K, T^{*}(K)$. But on $T^{*}(K)$ there is no distinguished class of functions that could play the role of $x_{k}$ and $p_{k}$ and thus tell us what the canonical commutation relations ought to be.

So if one considers general classical-mechanical systems, one will not have a preferred space of functions on the phase space that have simple relations under the Poisson bracket. As a result, when quantizing such systems, one will not have a simple set of commutation relations that could determine what the 
quantum operators should be. So instead of using commutation relations as our method of quantization we try some more geometrical construction of the quantum Hilbert space, which should have the property that if this construction is applied in the $\mathbb{R}^{d}$ case it produces one of the familiar quantizations of $\mathbb{R}^{d}$. In the $\mathbb{R}^{d}$ case, even though the classical phase space $\mathbb{R}^{2 d}$ is $2 d$-dimensional, the two (equivalent) possibilities we discussed for the quantum Hilbert space consist of spaces of functions of only $d$ variables. That is, in the "position" or "Schrödinger" representation $L^{2}\left(\mathbb{R}^{d}\right)$, our functions depend on the $d$ variables $x_{1}, \cdots, x_{d}$ but not on $p_{1}, \cdots, p_{d}$, and in the Segal-Bargmann space our functions depend on $z_{1}, \cdots, z_{d}$ but not on $\bar{z}_{1}, \cdots, \bar{z}_{d}$. We will consider similar possibilities in the group case. (More generally, the theory of geometric quantization [W] proceeds by choosing a "polarization" on a symplectic manifold, which is roughly a choice of $d$ variables out of $2 d$ on which the functions in the quantum Hilbert space should depend.)

In quantizing general classical systems we must simply accept that there are no canonical commutation relations. Even so, when constructing, say, a SegalBargmann transform, we should ask whether we have the "right" set-up. In the next subsection I will describe a version of the Segal-Bargmann transform for a compact Lie group, and after doing so I will discuss some things that seem "right" about this construction.

Before doing this, let me mention that there are some classical systems besides $\mathbb{R}^{2 d}$ which $d o$ have a distinguished set of functions that allow one to define something like the CCRs. (The cotangent bundle of a compact Lie group is not such a system.) Usually such functions arise in connection with some symmetry of the system. In the case of $\mathbb{R}^{2 d}$ the functions $x_{k}$ and $p_{k}$ have to do with the translational symmetry of $\mathbb{R}^{2 d}$. What this means is that if you consider Hamilton's equations with the Hamiltonian function $H(x, p)=p_{k}$, then the solutions are precisely the trajectories of the form

$$
\begin{aligned}
& x(t)=x_{0}+t e_{k} \\
& p(t)=p_{0},
\end{aligned}
$$

where $e_{k}$ is the vector $(0, \cdots, 0,1,0, \cdots 0)$, with the 1 in the $k$ th spot. This may be expressed as saying, " $p_{k}$ is the generator of translations in the $x_{k}$ direction." Similarly, $x_{k}$ generates translations in the negative $p_{k}$ direction.

More generally, we may consider a symplectic manifold $M$, that is, a manifold equipped with some reasonable notion of Poisson bracket (satisfying the same properties as in Exercise 23). If a Lie group $G$ acts transitively on $M$ in a way that preserves the Poisson bracket, then we may look for functions which "generate" the action of $G$ in the same way that the functions $x_{k}$ and $p_{k}$ generate the translational symmetries of $\mathbb{R}^{2 d}$. The collection of such functions is called the "moment map" for the action of $G$. It is then reasonable to take this collection of functions as our "basic functions." The general theory guarantees that these functions satisfy nice relations under the Poisson bracket, relations that are closely related to the commutation relations for the Lie algebra of $G$. So in this case the Poisson bracket relations among our basic functions give us a way of 
defining (generalized) canonical commutation relations. Even though we will not typically have a result like the Stone-von Neumann Theorem, there is in many cases a preferred way of building a quantum Hilbert space which satisfies the relevant commutation relations and the appropriate irreducibility condition.

The next simplest example (after $\mathbb{R}^{2 d}$ ) in which this scheme can be carried out is the unit disk, acted on by the group $S U(1,1)$ of fractional linear transformations that map the disk onto itself. There is a notion of Poisson bracket that is invariant under this action. The quantization of the disk by the above approach leads to our friends the weighted Bergman spaces, with the weight parameter $a$ being related to $\hbar$. The relevant commutation relations in this case are those of the Lie algebra of $S U(1,1)$. It is possible to exponentiate the corresponding operators to get the (projective) unitary representation of $S U(1,1)$ acting in the weighted Bergman spaces, as described in Section 4.2. One can similarly treat the unit ball in $\mathbb{C}^{d}$ and more generally bounded symmetric domains. See [KL1 and BLU] for an analysis of the Toeplitz quantization of these spaces.

A larger class of examples is that of co-adjoint orbits of Lie groups. The quantization of co-adjoint orbits gives an powerful method of constructing unitary representations of Lie groups, a method pioneered by A. Kirillov and B. Kostant, and since investigated in hundreds of papers. See the recent survey article $\mathrm{Ki}$.

\subsection{The transform for compact Lie groups}

I will concentrate on the simplest non-commutative example of a compact Lie group, even though the theory works in general. So let $K=\mathrm{SU}(2)$, the group of $2 \times 2$ unitary matrices with determinant one. Explicitly,

$$
\mathrm{SU}(2)=\left\{\left.\left(\begin{array}{rr}
\alpha & -\bar{\beta} \\
\beta & \bar{\alpha}
\end{array}\right)|\alpha, \beta \in \mathbb{C},| \alpha\right|^{2}+|\beta|^{2}=1\right\} .
$$

(See Exercise 2.) Note that $\mathrm{SU}(2)$ can be identified with the unit sphere $S^{3}$ inside $\mathbb{C}^{2}=\mathbb{R}^{4}$. In particular $\mathrm{SU}(2)$ is a compact manifold of (real) dimension 3 .

Now let $K_{\mathbb{C}}=\operatorname{SL}(2 ; \mathbb{C})$, the group of $2 \times 2$ matrices with determinant one, that is,

$$
\mathrm{SL}(2 ; \mathbb{C})=\left\{\left(\begin{array}{ll}
a & b \\
c & d
\end{array}\right) \mid a, b, c, d \in \mathbb{C}, a d-b c=1\right\} .
$$

Then $\operatorname{SL}(2 ; \mathbb{C})$ is a 3 -dimensional complex manifold, or a 6 -dimensional (noncompact) real manifold. Furthermore, $\operatorname{SU}(2)$ sits inside $\mathrm{SL}(2 ; \mathbb{C})$ in the same way that $\mathbb{R}^{3}$ sits inside $\mathbb{C}^{3}$, namely, as a "totally real submanifold of maximum dimension."

There is a natural Laplacian operator on $K$, namely, the spherical Laplacian, thinking of $\mathrm{SU}(2)$ as $S^{3} \subset \mathbb{R}^{4}$. This operator will be denoted $\Delta_{K}$. Geometrically, 
$\Delta_{K}$ is the Laplace-Beltrami operator with respect to a bi-invariant Riemannian metric on SU(2). I then want to consider the heat equation on $K$, namely,

$$
\frac{\partial u}{\partial t}=\frac{1}{2} \Delta_{K} u,
$$

where $u(x, t)$ is a function on $K \times(0, \infty)$. The equation is subject to an initial condition of the form

$$
\lim _{t \downarrow 0} u(x, t)=f(x) .
$$

We denote the (unique) solution to this equation schematically as

$$
u(x, t)=e^{t \Delta_{K} / 2} f,
$$

where $e^{t \Delta_{K} / 2}$ is the heat operator. That is, $e^{t \Delta_{K} / 2}$ is short-hand for the operator that associates to a function $f$ the solution at time $t$ of the heat equation with initial condition $f$. Note that formally the RHS of (51) satisfies the heat equation, and that formally at $t=0$ the RHS equals $f$. The expression $e^{t \Delta_{K} / 2}$ may be defined rigorously for example using the spectral theorem. However, even if the initial function $f$ is smooth, $e^{t \Delta_{K} / 2}$ cannot necessarily be computed by means of the power series for the exponential function.

The heat equation can be solved in the following way. We first find the heat kernel for $K$, which is the fundamental solution at the identity, denoted $\rho_{t}(x)$. This means that

$$
\frac{d \rho}{d t}=\frac{1}{2} \Delta_{K} \rho_{t}
$$

and

$$
\lim _{t \downarrow 0} \rho_{t}(x)=\delta_{e}(x) .
$$

Here $\delta_{e}(x)$ means a $\delta$-function at the identity. It is known that the heat kernel $\rho_{t}$ exists and is unique. In this case there is a fairly explicit formula for the heat kernel-see [H3].

Now let $d x$ denote the natural surface area measure on $\mathrm{SU}(2)=S^{3}$. In group-theoretical language $d x$ is the Haar measure for $\mathrm{SU}(2)$. Then (53) really means that for all continuous functions $f$,

$$
\lim _{t \downarrow 0} \int_{K} \rho_{t}(x) f(x) d x=f(e) .
$$

Once we have the heat kernel $\rho_{t}(x)$ and the Haar measure $d x$ we may express the heat operator $e^{t \Delta_{K} / 2}$ as follows:

$$
\left(e^{t \Delta_{K} / 2} f\right)(x)=\int_{K} \rho_{t}\left(x y^{-1}\right) f(y) d y .
$$

Here $x y^{-1}$ refers to product and inverse in the group SU(2). The RHS of (54) is a group-theoretical convolution of the function $\rho_{t}$ and the function $f$. 
Theorem 32 For each fixed $t>0$ the heat kernel $\rho_{t}(x)$ has a unique analytic continuation from $K=\mathrm{SU}(2)$ to $K_{\mathbb{C}}=\mathrm{SL}(2 ; \mathbb{C})$.

Note that here we are analytically continuing in the space variable $x$, which initially lived in $\mathrm{SU}(2)$ but is now extended by analytic continuation to $\mathrm{SL}(2 ; \mathbb{C})$. I will continue to call the holomorphic function obtained by this analytic continuation $\rho_{t}$. Let $\mathcal{H}\left(K_{\mathbb{C}}\right)$ denote the space of (entire) holomorphic functions on $K_{\mathbb{C}}=\mathrm{SL}(2 ; \mathbb{C})$. Then we are now ready to define the generalized SegalBargmann transform for $K$. We will now let Planck's constant $\hbar$ play the role of time in the heat equation.

Definition 33 For each $\hbar>0$, define a map

$$
C_{\hbar}: L^{2}(K, d x) \rightarrow \mathcal{H}\left(K_{\mathbb{C}}\right)
$$

by

$$
\left(C_{\hbar} f\right)(g)=\int_{K} \rho_{\hbar}\left(g x^{-1}\right) f(x) d x, \quad g \in K_{\mathbb{C}} .
$$

Here $\rho_{\hbar}$ refers to the analytically continued heat kernel, and $g x^{-1}$ refers to the product of the element $g \in \mathrm{SL}(2 ; \mathbb{C})$ and the element $x^{-1} \in \mathrm{SU}(2) \subset$ $\mathrm{SL}(2 ; \mathbb{C})$. Because of the analytic continuation it makes sense to plug an element of $\operatorname{SL}(2 ; \mathbb{C})$ into $\rho_{\hbar}$. Since $\rho_{\hbar}(g)$ is holomorphic (by construction) as a function of $g$, it is easily seen that $\rho_{\hbar}\left(g x^{-1}\right)$ is holomorphic as a function of $g$ for each fixed $x$. It then follows that $C_{\hbar} f(g)$ is holomorphic as a function of $g$ for any $f \in L^{2}(K, d x)$. If we restrict our attention to $g \in K$, then we recognize $C_{\hbar} f$ as $e^{\hbar \Delta_{K} / 2} f$. Thus we may also write

$$
C_{\hbar} f=\text { analytic continuation of } e^{\hbar \Delta_{K} / 2} f .
$$

Again the analytic continuation is in the space variable, from $K=\mathrm{SU}(2)$ to $K_{\mathbb{C}}=\mathrm{SL}(2 ; \mathbb{C})$.

Theorem 34 For each $\hbar>0$ there exists a measure $\nu_{\hbar}$ on $K_{\mathbb{C}}$ such that $C_{\hbar}$ is a unitary map from $L^{2}(K, d x)$ onto $\mathcal{H} L^{2}\left(K_{\mathbb{C}}, \nu_{\hbar}\right)$.

Let us see the analogy between this generalized Segal-Bargmann transform for $\mathrm{SU}(2)$ and the $C_{\hbar}$ version of the Segal-Bargmann transform for $\mathbb{R}^{d}$, as described in Theorem 21. We may think of $\mathbb{R}^{d}$ as a commutative group under addition. In that case we may recognize the first expression in Theorem 21 as the convolution of $\rho_{\hbar}$ with the function $f$ (with the group operation now written in additive notation). Furthermore it may be verified by direct calculation (or by consulting a standard text on partial differential equations) that the function

$$
\rho_{t}(x)=(2 \pi t)^{-d / 2} e^{-x^{2} / 2 t}
$$

is the heat kernel for $\mathbb{R}^{d}$. (That is, it satisfies the heat equation and concentrates to a $\delta$-function at the origin as $t \downarrow 0$.) Thus if we substitute the group $\mathbb{R}^{d}$ for 
the group SU(2) in Definition 33 we recover precisely the $C_{\hbar}$ version of the Segal-Bargmann transform for $\mathbb{R}^{d}$.

The measure $\nu_{\hbar}$ should be the group-theoretical analog of the measure $d \nu_{\hbar}(z):=(\pi \hbar)^{-d / 2} \exp \left(-(\operatorname{Im} z)^{2} / \hbar\right) d z$ on $\mathbb{C}^{d}$. To see how to make this analogy we may observe that in the $\mathbb{C}^{d}$ case the density of the measure $\nu_{\hbar}$ satisfies the heat equation on $\mathbb{C}^{d}=\mathbb{R}^{2 d}$ (check!). Furthermore this density is independent of $x=\operatorname{Re} z$. Similarly, the measure $\nu_{\hbar}$ on $K_{\mathbb{C}}$ has a density (with respect to the natural Haar measure on $K_{\mathbb{C}}$ ) that satisfies a suitable heat equation on $K_{\mathbb{C}}$ and that is invariant under the action of $K$. (The invariance means that the density satisfies $\nu_{\hbar}(g x)=\nu_{\hbar}(g)$ for all $g \in K_{\mathbb{C}}$ and all $x \in K$.)

Remarks. 1) There is also a version of the generalized Segal-Bargmann transform for $K$ that is precisely analogous to the $B_{\hbar}$ form of the Segal-Bargmann transform for $\mathbb{R}^{d}$. (That is, precisely analogous to the $B_{\hbar}$ transform as I have described it, which differs by some factors of $\sqrt{2}$ from what Segal describes.) As in the $\mathbb{R}^{d}$ case, the formula for the transform $B_{\hbar}$ is the same as the formula for $C_{\hbar}$, but the measures on $K$ and $K_{\mathbb{C}}$ are different in the two cases. For $B_{\hbar}$ the measure on $K$ is the heat kernel measure $\rho_{\hbar}(x) d x$, and the measure on $K_{\mathbb{C}}$ is the full heat kernel measure $\mu_{t}(g) d g$. The $B_{\hbar}$ transform is described in Theorem $1^{\prime}$ of [H1]. In contrast to the $\mathbb{R}^{d}$ case, the two transforms for $K$ are not "equivalent." That is, there is no change-of-variable on $K$ like the one on $\mathbb{R}^{d}$ that converts one transform into the other. In the $\mathbb{R}^{d}$ case the two transforms are interchangeable, really just two different normalizations of the same transform. (Cf. Section 3 of [H5].) In the group case the two transforms are genuinely distinct, and the $C_{\hbar}$ version seems to be better-behaved, in part because it respects the symmetry of left- and right-translations by $K$.

2) The generalized Segal-Bargmann transform can be constructed in a precisely analogous way for an arbitrary connected compact Lie group $K$ (with a fixed bi-invariant Riemannian metric). One defines the complexification $K_{\mathbb{C}}$ of $K$ and the Laplacian $\Delta_{K}$, and everything goes through exactly as above. I have restricted to the case $K=\mathrm{SU}(2)$ merely to keep the discussion as concrete and elementary as possible.

\subsection{What is "right" about this transform?}

We have already observed that when moving beyond the setting of $\mathbb{R}^{d}$ we cannot expect to have a nice analog of the canonical commutation relations. Nevertheless, we want to have some way of deciding when we have the "right" definition of the Segal-Bargmann transform for a compact Lie group. That is, why this transform and not some other? We have already seen two good things about the transform $C_{\hbar}: 1$ ) it is unitary, and 2) when the group $\mathrm{SU}(2)$ is replaced by the group $\mathbb{R}^{d}$ we get back precisely the $C_{\hbar}$ form of the Segal-Bargmann transform for $\mathbb{R}^{d}$. I want to describe several additional aspects of this transform that suggest it is in some sense "right." Of course this does not preclude the possibility of some other useful Segal-Bargmann transform for SU(2) or some other group. (Indeed C. Villegas has introduced a different transform for $S^{3}=\mathrm{SU}(2)$ which might be preferable in connection with the study of the Kepler problem.) 
The complex group as phase space. If we are to think of the Segal-Bargmann transform for $K=\mathrm{SU}(2)$ in the same way we think of the Segal-Bargmann transform for $\mathbb{R}^{d}$, then we want to think of the complex group $K_{\mathbb{C}}=\mathrm{SL}(2 ; \mathbb{C})$ as the phase space corresponding to the configuration space $K=\mathrm{SU}(2)$. On the other hand, in classical mechanics, if a given manifold $X$ is the configuration space, then the phase space is usually taken to be the cotangent bundle of $X$, $T^{*}(X)$. So we would like to be able to identify the complex group $K_{\mathbb{C}}$ with $T^{*}(K)$. This may be done as follows. We introduce the Lie algebra su $(2)$ of SU(2). By definition, the Lie algebra is the set of all $2 \times 2$ matrices $Y$ such that $\exp t Y$ lies in $\mathrm{SU}(2)$ for all real $t$, where exp is the matrix exponential and is computed as a (convergent) power series. It is not to hard to show (Exercise 5) that $\mathrm{su}(2)$ is given explicitly as

$$
\operatorname{su}(2)=\left\{2 \times 2 \text { matrices } Y \mid Y^{*}=-Y \text { and } \operatorname{trace}(Y)=0\right\} .
$$

This is a 3 -dimensional real vector space. Geometrically, su(2) can be identified with the tangent space at the identity to $\mathrm{SU}(2)$. We may similarly define the Lie algebra $\mathrm{sl}(2 ; \mathbb{C})$ of $\mathrm{SL}(2 ; \mathbb{C})$. Explicitly $\mathrm{sl}(2 ; \mathbb{C})$ may be computed as the space of all $2 \times 2$ matrices with trace zero-a 3 -dimensional complex vector space. Note that $\mathrm{sl}(2 ; \mathbb{C})=\operatorname{su}(2) \oplus i \mathrm{su}(2)$.

Now using the left action of $\mathrm{SU}(2)$ on itself, the cotangent bundle of $\mathrm{SU}(2)$ can be trivialized. Thus $T^{*}(K)$ is diffeomorphic to $\mathrm{SU}(2) \times \mathrm{su}(2)^{*}$. Using the natural inner product on su(2), we may identify su(2) with su(2)*, so that $T^{*}(K)$ is identified with $\mathrm{SU}(2) \times \mathrm{su}(2)$. We then make use of the map

$$
\Phi: \operatorname{SU}(2) \times \operatorname{su}(2) \rightarrow \operatorname{SL}(2 ; \mathbb{C})
$$

given by

$$
\Phi(x, Y)=x \exp i Y, \quad x \in \mathrm{SU}(2), Y \in \operatorname{su}(2) .
$$

It turns out that $\Phi$ is a diffeomorphism of $T^{*}(\operatorname{SU}(2))$ onto $\operatorname{SL}(2 ; \mathbb{C})$. Note here that $Y$ is skew-adjoint with trace zero, so that $i Y$ is self-adjoint with trace zero. It follows that $\exp i Y$ is self-adjoint and positive with determinant one. So in order to express an arbitrary matrix $g$ in $\operatorname{SL}(2 ; \mathbb{C})$ as $\Phi(x, Y)$, we use the polar decomposition to express $g$ as $g=x p$, with $x$ unitary with determinant one and $p$ self-adjoint and positive with determinant one. Then $i Y$ is the unique self-adjoint logarithm of $p$.

The diffeomorphism $\Phi$ between $T^{*}(\mathrm{SU}(2))$ and $\mathrm{SL}(2 ; \mathbb{C})$ is in a certain sense canonical-see [H3] or [H4]. In particular the complex structure of $\mathrm{SL}(2 ; \mathbb{C})$ and the symplectic structure of $T^{*}(\mathrm{SU}(2))$ fit together so as to form a Kähler manifold. So indeed there is a natural way of identifying $K_{\mathbb{C}}=\mathrm{SL}(2 ; \mathbb{C})$ with the phase space over $K=\mathrm{SU}(2)$. This shows the Segal-Bargmann transform for $K$ described above is reasonable. This identification of $T^{*}(K)$ with $K_{\mathbb{C}}$ works in a similar way for any compact Lie group $K$.

Additional results. Some of what is known about the transform for $K$, beyond unitarity, seems to suggest that it behaves the way a Segal-Bargmann transform 
ought to behave. For example, [H2] gives a very natural inversion formula for $C_{\hbar}$, which says roughly that the "position wave function" $f(x)$ can be recovered from the "phase space wave function" $C_{\hbar} f$ by integrating out the momentum variables. This is the group analog of the first part of Theorem 31. Further, H3 gives physically natural (and non-obvious) phase space bounds on the transform of an arbitrary function $f$, namely, a group version of Exercise 29 of the previous section.

Alternative constructions of the generalized Segal-Bargmann transform. I want to describe briefly two additional constructions that turn out to produce precisely the same generalized Segal-Bargmann transform for SU(2) (or for any compact Lie group $K$ ). So altogether there are three very different constructions that all produce exactly the same transform, which suggests that there is something right about this transform.

The first alternative approach was proposed by L. Gross and P. Malliavin [GM], who derived the $B_{\hbar}$ form of the generalized Segal-Bargmann transform for a compact Lie group $K$ from the infinite-dimensional ordinary Segal-Bargmann transform. By modifying the approach of Gross and Malliavin, Bruce Driver and I [DH] derived the $C_{\hbar}$ form of the transform for $K$ from the infinite-dimensional classical transform. The idea is to start with a certain infinite-dimensional linear space $\mathcal{A}$ and then to "reduce" by a certain action of the loop group over $K$. (See also [H6, HS].) This reduction turns $\mathcal{A}$ into a single copy of the compact Lie group $K$, and it turns the ordinary Segal-Bargmann transform for $\mathcal{A}$ into the generalized Segal-Bargmann transform for $K$. Of course it was not obvious ahead of time that doing the Segal-Bargmann transform for $\mathcal{A}$ and then reducing down to $K$ would give the same result as doing the generalized Segal-Bargmann transform for $K$.

The second alternative approach to the Segal-Bargmann transform for $K$ is that of geometric quantization. Geometric quantization [W] aims to associate in as canonical a way as possible to a symplectic manifold $M$ (the classical phase space) a Hilbert space $\mathcal{H}$ and to functions on $M$ operators in the Hilbert space $\mathcal{H}$. It is generally accepted that quantization cannot be done without some additional structure on $M$; in geometric quantization this additional structure is taken to be a "polarization" on $M$. Roughly speaking, a polarization means a choice of $d$ variables out of the $2 d$ variables on $M$ on which the functions in the quantum Hilbert space should depend. So in the case of a system with configuration space $\mathbb{R}^{d}$ and phase space $\mathbb{R}^{2 d}$ we have seen two possibilities for the quantum Hilbert space, $L^{2}\left(\mathbb{R}^{d}\right)$ and the Segal-Bargmann space. In $L^{2}\left(\mathbb{R}^{d}\right)$ we have functions that depend on the position variables $x_{1}, \cdots, x_{d}$ but are independent of the momentum variables $p_{1}, \cdots, p_{d}$. In the Segal-Bargmann space we have functions that depend on $z_{1}, \cdots, z_{d}$ but are independent of $\bar{z}_{1}, \cdots, \bar{z}_{d}$ (in the sense that $\partial F / \partial \bar{z}_{k}=0$ ).

On the cotangent bundle of a compact Lie group $K$ we have two natural polarizations. The first is the "vertical polarization," which makes sense for any cotangent bundle. Here we take the coordinates on $K$ itself as the ones on which our functions depend, and we take the coordinates in the cotangent spaces as the ones on which our functions will not depend. In terms of the decomposition 
$T^{*}(\mathrm{SU}(2))=\mathrm{SU}(2) \times \operatorname{su}(2)$, we want functions that depend on the "position" variable $x \in \mathrm{SU}(2)$ but not on the "momentum" variable $Y \in \mathrm{su}(2)$. The second polarization is a complex polarization (or "Kähler polarization") which comes from the identification of $T^{*}(\mathrm{SU}(2))$ with $\mathrm{SL}(2 ; \mathbb{C})$. This identification makes $T^{*}(\mathrm{SU}(2))$ into a complex manifold, and so it makes sense to speak of functions that in holomorphic local coordinates depend on $z_{1}, z_{2}, z_{3}$ but not on $\bar{z}_{1}, \bar{z}_{2}, \bar{z}_{3}$.

Now in geometric quantization the Hilbert space is not actually a space of functions, but rather a space of sections of a complex line bundle over the phase space $M$. These sections are required to be "covariantly constant" in the direction of the polarization. It takes some time to unravel what all this really means, but when the shouting and tumult have died down we have the following result. Using the vertical polarization on $T^{*}(K)$ the quantum Hilbert space may be identified with $L^{2}(K)$. (The space of sections of the line bundle gets identified with a space of functions by trivializing the line bundle.) Using the complex polarization the quantum Hilbert space may be identified with an $L^{2}$ space of holomorphic functions on $K_{\mathbb{C}}$, with respect to a certain measure. It turns out that the measure coming from geometric quantization coincides exactly (up to an irrelevant overall constant) with the measure $\nu_{\hbar}$ that appears in the generalized Segal-Bargmann space. (More precisely, this is true provided that one includes the "half-form correction" in the geometric quantization. Cf. Sect. 7 of [H4] in which I consider the geometric quantization without the half-form correction.) See [H7].

Thus the process of geometric quantization reproduces the generalized SegalBargmann space over $K_{\mathbb{C}}$. Not only so, but geometric quantization also reproduces the Segal-Bargmann transform for $K$. That is, there is in geometric quantization something called the "pairing map." The pairing map is a map between the quantum Hilbert spaces constructed using two different polarizations. In general the pairing map need not be unitary. However, in the case of the pairing map between the vertically polarized Hilbert space over $K$ and the complex-polarized Hilbert space, the pairing map is unitary and coincides precisely with the generalized Segal-Bargmann transform. (All of this holds for an arbitrary compact Lie group $K$ and its complexification $K_{\mathbb{C}}$.)

It is a seeming miracle that geometric quantization should reproduce the generalized Segal-Bargmann space and transform. After all, the Segal-Bargmann space and transform were defined in terms of heat kernels, and geometric quantization seems to have nothing to do with heat kernels or the heat equation. Clearly something very special is going on in this example that deserves to be understood better.

We have, then, three completely different constructions of the Segal-Bargmann transform for a compact Lie group $K$. The first construction is in terms of heat kernels, the second is by reduction from an infinite-dimensional linear space, and the third is by geometric quantization. That all three constructions yield the same transform suggests that we are doing something right.

In a more general setting, say in which the compact Lie group is replaced by a more general Riemannian manifold $X$, it is unlikely that all three of these constructions will give the same answer. I hope that having these three different 
approaches will give sufficient insight that one can see how to construct a wellbehaved Segal-Bargmann transform for some more general class of manifolds $X$. Time will tell!

\subsection{Exercises}

1. * Verify that the expression

$$
\left\{f_{1}, f_{2}\right\}=-4 i\left(1-|z|^{2}\right)^{2}\left(\frac{\partial f_{1}}{\partial z} \frac{\partial f_{2}}{\partial \bar{z}}-\frac{\partial f_{1}}{\partial \bar{z}} \frac{\partial f_{2}}{\partial z}\right)
$$

defines a Poisson bracket on the unit disk $\mathbb{D}$ that is invariant under the action of $\operatorname{SU}(1,1)$.

2. Verify that every element of the form (48) is really unitary and has determinant one, and that every $2 \times 2$ unitary matrix with determinant one can be expressed in this form.

3. Verify that the function

$$
\rho_{t}(x)=(2 \pi t)^{-d / 2} e^{-x^{2} / 2 t}
$$

on $\mathbb{R}^{d}$ satisfies the heat equation and that for every continuous compactly supported function $f$ on $\mathbb{R}^{d}$,

$$
\lim _{t \downarrow 0} \int_{\mathbb{R}^{d}} \rho_{t}(x) f(x) d x=f(0) .
$$

4. * a) Show that every element $g$ of $\operatorname{SL}(2 ; \mathbb{C})$ can be written in the form

$$
g=x_{1} e^{a H} x_{2}
$$

with $a \in \mathbb{R}$, and $x_{1}, x_{2} \in \mathrm{SU}(2)$. Here

$$
H=\left(\begin{array}{cc}
1 & 0 \\
0 & -1
\end{array}\right) .
$$

Hint: first write $g=x \exp p$ with $x \in \mathrm{SU}(2)$ and $p$ self-adjoint with trace zero. Then diagonalize $p$.

b) Consider the series expansion for the heat kernel on SU(2) (cf. Eq. (11) of [H1])

$$
\rho_{t}(x)=\sum_{l}(2 l+1) e^{-t l(l+1) / 2} \operatorname{trace}\left(\pi_{l}(x)\right),
$$

where $\pi_{l}$ is the irreducible representation of SU(2) of dimension $2 l+1$, and where $l=0,1 / 2,1,3 / 2,2, \cdots$. We want to analytically continue $\rho_{t}$ from $\mathrm{SU}(2)$ to $\mathrm{SL}(2 ; \mathbb{C})$ by analytically continuing $(57)$ term-by-term. Show using (56) that the analytically continued series converges uniformly on compact subsets of $\mathrm{SL}(2 ; \mathbb{C})$. (Cf. Sect. 4 of [H1].) This shows that $\rho_{t}$ admits an analytic continuation from $\mathrm{SU}(2)$ to $\mathrm{SL}(2 ; \mathbb{C})$. Hint: what are the eigenvalues of $H$ in the representation $\pi_{l}$ ?

5. Verify the description (55) of the Lie algebra su (2) of SU(2). 


\section{To infinity and beyond}

In this section I will touch briefly on a few additional topics, to give the flavor of them and to suggest directions for further reading.

\subsection{The infinite-dimensional theory}

I have already mentioned that Segal wished to consider systems with infinitely many degrees of freedom, describing quantum field theory instead of ordinary quantum mechanics. This means that Segal wanted to let the dimension $d$ tend to infinity. This limit raises several interesting technical issues. Most important, there is no such thing as Lebesgue measure on an infinite-dimensional space. Thus the ground state transformation, leading to the $B_{\hbar}$ form of the SegalBargmann transform, is essential when $d=\infty$.

So consider a real Hilbert space $X_{\mathbb{R}}$, which I assume is infinite-dimensional and separable. We think of this as the $d \rightarrow \infty$ limit of $\mathbb{R}^{d}$. We need to try to construct the appropriate measure on $\rho_{\hbar}$ on $X_{\mathbb{R}}$, which should be the infinite-dimensional limit of the measures appearing in the $B_{\hbar}$ form of the SegalBargmann transform for $\mathbb{R}^{d}$. So we might imagine picking an increasing sequence of finite-dimensional subspaces $V_{d}$ of $X_{\mathbb{R}}$, with $\operatorname{dim} V_{d}=d$ and chosen so that the union of the $V_{d}$ 's is dense in $X_{\mathbb{R}}$. Then we may consider a sequence of measures $\rho_{\hbar}^{(d)}$ on $X_{\mathbb{R}}$ such that $\rho_{\hbar}^{(d)}$ is concentrated on $V_{d}$ and given by

$$
d \rho_{\hbar}^{(d)}(x)=(2 \pi \hbar)^{-d / 2} e^{-\|x\|^{2} / 2 \hbar} d x
$$

where $d x$ is the Lebesgue measure on $V_{d}$ and the constant in front normalizes $\rho_{\hbar}^{(d)}$ to be a probability measure. We then need to let $d$ tend to infinity.

Unfortunately, the limit $\lim _{d \rightarrow \infty} \rho_{\hbar}^{(d)}$ does not exist as a measure on $X_{\mathbb{R}}$. To see intuitively why this is so, first consider the two-dimensional case. The measure $\rho_{\hbar}$ on $\mathbb{R}^{2}$ is given explicitly as

$$
\begin{aligned}
d \rho_{\hbar}(x, y) & =(2 \pi \hbar)^{-1} e^{-\left(x^{2}+y^{2}\right) / 2 \hbar} d x d y \\
& =\left[(2 \pi \hbar)^{-1 / 2} e^{-x^{2} / 2 \hbar} d x\right]\left[(2 \pi \hbar)^{-1 / 2} e^{-y^{2} / 2 \hbar} d y\right] .
\end{aligned}
$$

Note that the measure factors as a measure in the $x$ variable times a measure in the $y$ variable, both of which are probability measures, in fact, the same probability measure. We may say the same thing in probabilistic language by saying that (with respect to $\rho_{\hbar}$ ) $x$ and $y$ are independent and identically distributed. We may recognize the distribution of $x$ or $y$ as normal with mean zero and variance $\hbar$. The same sort of product decomposition will hold for the measures $\rho_{\hbar}^{(d)}$ in every dimension $d$. So now suppose that the $\rho_{\hbar}^{(d)}$ 's did converge to a probability measure $\rho_{\hbar}$, and let $\left\{e_{i}\right\}$ be an orthonormal basis for $X_{\mathbb{R}}$ and $\left\{x_{i}\right\}$ the coordinates with respect to this basis. Then the coordinates $x_{i}$ would presumably be independent, with each $x_{i}$ normal with mean zero and variance $\hbar$. But if a vector $v$ is in $X_{\mathbb{R}}$ then the coordinates $x_{i}$ of that vector 
satisfy $\Sigma x_{i}^{2}=\|v\|^{2}<\infty$. On the other hand, if $\left\{x_{i}\right\}$ are independent normal random variables with mean zero and all having the same variance $\hbar$, then it is intuitively obvious that $\Sigma x_{i}^{2}=\infty$ with probability one. So roughly speaking the points of finite norm in $X_{\mathbb{R}}$ (that is, all of $X_{\mathbb{R}}$ !) constitute a set of measure zero. So $\rho_{\hbar}$ cannot be a probability measure on $X_{\mathbb{R}}$.

Even though $\rho_{\hbar}$ does not exist as a measure on $X_{\mathbb{R}}$, it should exist as a measure on something. After all, it is possible to have an infinite sequence of independent random variables with mean zero and variance $\hbar$. Following the approach of $[\mathrm{Gr}]$ we consider a certain "extension" of $X_{\mathbb{R}}$, denoted $\bar{X}_{\mathbb{R}}$. By this I mean that $\bar{X}_{\mathbb{R}}$ is a Banach space and that there is a continuous embedding of the Hilbert space $X_{\mathbb{R}}$ into $\bar{X}_{\mathbb{R}}$. If $\bar{X}_{\mathbb{R}}$ is sufficiently much larger than $X_{\mathbb{R}}$, then in a natural way $\rho_{\hbar}$ may be regarded as a measure on $\bar{X}_{\mathbb{R}}$. The resulting measure $\rho_{\hbar}$ is called a Gaussian measure on $\bar{X}_{\mathbb{R}}$, and the subspace $X_{\mathbb{R}} \subset \bar{X}_{\mathbb{R}}$ is called the Cameron-Martin subspace. The Cameron-Martin subspace is a set of measure zero with respect to $\rho_{\hbar}$. (See also [Ku].)

The prototypical example is the following. We take $X_{\mathbb{R}}$ to be the space of absolutely continuous functions $B:[0,1] \rightarrow \mathbb{R}$ such that 1) $B(0)=0$ and 2) $\int_{0}^{1}|d B / d t|^{2} d t<\infty$, with inner product given by

$$
\left\langle B_{1}, B_{2}\right\rangle=\int_{0}^{1} \frac{d B_{1}}{d t} \frac{d B_{2}}{d t} d t .
$$

We take $\bar{X}_{\mathbb{R}}$ to be $\mathcal{C}_{0}([0,1])$, that is, the space of continuous functions $B$ : $[0,1] \rightarrow \mathbb{R}$ such that $B(0)=0$. Then for each $\hbar>0$ there exists a well-defined measure $\rho_{\hbar}$ on $\mathcal{C}_{0}([0,1])$ that may be thought of as the infinite-dimensional limit of the measures $\rho_{\hbar}^{(d)}$ on $X_{\mathbb{R}}$. The measure $\rho_{\hbar}$ has the highly non-rigorous formal expression

$$
d \rho_{\hbar}(B)=\text { const. } \exp \left[-\frac{1}{2 \hbar} \int_{0}^{1}\left|\frac{d B}{d t}\right|^{2} d t\right] \mathcal{D} B,
$$

where $\mathcal{D} B$ is the non-existent Lebesgue measure on $\mathcal{C}_{0}([0,1])$ and the constant is supposed to normalize $\rho_{\hbar}$ to be a probability measure. Note that the expression in the exponent is just $-\|B\|^{2} / 2 \hbar$, where the norm is computed using the inner product (58). Even though the measure lives on $\mathcal{C}_{0}([0,1])$, the properties of the measure are determined by the norm on $X_{\mathbb{R}}$. Expressions of the form (59) are common in the physics literature.

The measure described in the previous paragraph is the Wiener measure. As a measure on the space of continuous paths, it describes the behavior of Brownian motion. The typical path $B$ (with respect to the measure $\rho_{\hbar}$ ) is very wiggly and non-differentiable. A general triple $\left(X_{\mathbb{R}}, \bar{X}_{\mathbb{R}}, \rho_{\hbar}\right)$ of the sort considered above is called an abstract Wiener space, in honor of the motivating example of the Wiener measure. (The terminology is due to Gross [Gr].)

We have then a good candidate for the domain Hilbert space of our SegalBargmann transform in the infinite-dimensional case, namely, $L^{2}\left(\bar{X}_{\mathbb{R}}, \rho_{\hbar}\right)$. We 
now need to find the right range Hilbert space. So consider the complexified Hilbert space $X_{\mathbb{C}}=X_{\mathbb{R}}+i X_{\mathbb{R}}$, the family $V_{d}^{\mathbb{C}}=V_{d}+i V_{d}$ of finite-dimensional subspaces, and the family $\mu_{\hbar}^{(d)}$ of measures given by

$$
d \mu_{\hbar}^{(d)}(z)=(\pi \hbar)^{-d} e^{-\|z\|^{2} / \hbar} d z,
$$

where $d z$ is Lebesgue measure on $V_{d}^{\mathbb{C}}$. One can consider the limit $\mu_{\hbar}$ of these measures, which exists as a measure on a certain extension $\bar{X}_{\mathbb{C}}$ of $X_{\mathbb{C}}$, where $\bar{X}_{\mathbb{C}}$ is a complex Banach space. (As on the domain side, we have $\mu_{\hbar}\left(X_{\mathbb{C}}\right)=0$.) In the case of the Wiener measure, $\bar{X}_{\mathbb{C}}$ may be taken to be the space of continuous functions $Z:[0,1] \rightarrow \mathbb{C}$ with $Z(0)=0$.

Now there exists a perfectly suitable notion of what it means for a function on a complex Banach space such as $\bar{X}_{\mathbb{C}}$ to be holomorphic, and so it seems plausible that we should take the Segal-Bargmann space to be the space of holomorphic functions on $\bar{X}_{\mathbb{C}}$ that are square-integrable with respect to $\mu_{\hbar}$. Unfortunately, this definition does not work, because in the infinite-dimensional case the space of square-integrable holomorphic functions is not a closed subspace of $L^{2}\left(\bar{X}_{\mathbb{C}}, \mu_{\hbar}\right)$ and therefore not a Hilbert space. There are then two approaches to defining the Segal-Bargmann space. The first approach is essentially to define the Segal-Bargmann space to be the closure in $L^{2}\left(\bar{X}_{\mathbb{C}}, \mu_{\hbar}\right)$ of the space of holomorphic functions. This is the approach used in [HS, DH]. (See also $\mathrm{Sh}, \mathrm{Su}$.)

Another approach to the Segal-Bargmann space is to consider holomorphic functions on $X_{\mathbb{C}}$ itself. In that case the $L^{2}$ norm is meaningless (since the measure $\mu_{\hbar}$ is not defined on $X_{\mathbb{C}}$ ), but we can define a norm as follows. Suppose $F$ is a holomorphic function on $X_{\mathbb{C}}$. Then define $\|F\|_{\hbar}$ by

$$
\|F\|_{\hbar}^{2}=\sup _{d} \int_{V_{d}^{\mathbb{C}}}|F(z)|^{2} d \mu_{\hbar}^{(d)}(z),
$$

where the $V_{d}^{\mathbb{C}}$ 's are the finite-dimensional subspaces introduced above. The Segal-Bargmann space is then defined to be

$$
\mathcal{H}^{\hbar}\left(X_{\mathbb{C}}\right)=\left\{F: \mathcal{H}_{\mathbb{C}} \rightarrow \mathbb{C} \mid F \text { is holomorphic and }\|F\|_{\hbar}<\infty\right\} .
$$

It turns out that $\|F\|_{\hbar}$ is a norm on $\mathcal{H}^{\hbar}\left(X_{\mathbb{C}}\right)$ and that $\mathcal{H}^{\hbar}\left(X_{\mathbb{C}}\right)$ becomes a Hilbert space if we take the inner product to be

$$
\left\langle F_{1}, F_{2}\right\rangle=\sup _{d} \int_{V_{d}^{\complement}} \overline{F_{1}(z)} F_{2}(z) d \mu_{\hbar}^{(d)}(z) .
$$

This is the form of the Segal-Bargmann space used in S3, BSZ (except that Segal always uses anti-holomorphic rather than holomorphic functions).

We now state a form of the Segal-Bargmann theorem for the infinite-dimensional case. 
Theorem 35 For all $f \in L^{2}\left(\bar{X}_{\mathbb{R}}, \rho_{\hbar}\right)$ there exists a unique holomorphic function $B_{\hbar} f$ on $X_{\mathbb{C}}$ whose restriction to $X_{\mathbb{R}}$ is given by

$$
B_{\hbar} f(y)=\int_{\bar{X}_{\mathbb{R}}} f(y-x) d \rho_{\hbar}(x), \quad y \in X_{\mathbb{R}} .
$$

(The integral is well-defined and convergent for all $y \in X_{\mathbb{R}}$.) Furthermore, $B_{\hbar}$ is a unitary map of $L^{2}\left(\bar{X}_{\mathbb{R}}, \rho_{\hbar}\right)$ onto $\mathcal{H}^{\hbar}\left(X_{\mathbb{C}}\right)$.

Remarks. 1) Note that we compute the value of $B_{\hbar} f$ directly on $X_{\mathbb{R}}$ by the integral (60). To get the value on $X_{\mathbb{C}}$ we analytically continue from $X_{\mathbb{R}}$ to $X_{\mathbb{C}}$.

2) A simple change of variable shows that in the finite-dimensional case the transform defined here agrees with that of Section 6. Once one knows a reasonable amount about Gaussian measure spaces and about the space $\mathcal{H}^{\hbar}\left(X_{\mathbb{C}}\right)$, the proof is a straightforward reduction to the finite-dimensional case.

3) To verify that the integral in the theorem makes sense one needs to know that the measure $\rho_{\hbar}$ is "quasi-invariant" under translations in the direction of $X_{\mathbb{R}}$. (This quasi-invariance is the content of the Cameron-Martin Theorem.)

4) Theorem 35 is similar to Proposition 4.7 and Theorem 4.8 of GM. (Cf. Corollary 11 of [HS].)

\subsection{Coherent states}

In a holomorphic $L^{2}$ space, the coherent states are the unique elements $\phi_{z} \in$ $\mathcal{H} L^{2}(U, \alpha)$ such that

$$
F(z)=\left\langle\phi_{z}, F\right\rangle
$$

for all $F \in \mathcal{H} L^{2}(U, \alpha)$. (In the standard lingo of quantum physics a "state" means simply a non-zero element of the relevant Hilbert space.) The states $\phi_{z}$ are the same as those in Section 2. That is, the coherent states are given by

$$
\phi_{z}(w)=\overline{K(z, w)},
$$

where $K(z, w)$ is the reproducing kernel. Using the basic property (61) of the $\phi_{z}$ 's we see that the reproducing kernel is just the inner product of the coherent states:

$$
K(z, w)=\left\langle\phi_{z}, \phi_{w}\right\rangle .
$$

Meanwhile, let's consider $L^{2}\left(\mathbb{R}^{d}, d x\right)$ and the "invariant" form $C_{\hbar}$ of the Segal-Bargmann transform. (A similar analysis can be done with the other forms.) We now want to define coherent states $\psi_{z}$ in $L^{2}\left(\mathbb{R}^{d}, d x\right)$. These are the unique states $\psi_{z} \in L^{2}\left(\mathbb{R}^{d}, d x\right)$ such that

$$
C_{\hbar} f(z)=\left\langle\psi_{z}, f\right\rangle_{L^{2}\left(\mathbb{R}^{d}, d x\right)} .
$$

Since the Segal-Bargmann transform is unitary, we have

$$
C_{\hbar} f(z)=\left\langle\psi_{z}, f\right\rangle_{L^{2}\left(\mathbb{R}^{d}, d x\right)}=\left\langle C_{\hbar} \psi_{z}, C_{\hbar} f\right\rangle_{\mathcal{H} L^{2}\left(\mathbb{C}^{d}, \nu_{\hbar}\right)} .
$$


Comparing this with (61) we see that

$$
C_{\hbar} \psi_{z}=\phi_{z} .
$$

So if you prefer you may define the coherent states in $L^{2}\left(\mathbb{R}^{d}, d x\right)$ by

$$
\psi_{z}=C_{\hbar}^{-1} \phi_{z}
$$

From (63) and (65) we see that $K(z, w)=\left\langle\psi_{z}, \psi_{w}\right\rangle$.

Recalling the formula for the $C_{\hbar}$ form of the Segal-Bargmann transform we see that the states satisfying (64) are

$$
\psi_{z}(x)=(2 \pi \hbar)^{-d / 2} e^{-(\bar{z}-x)^{2} / 2 \hbar} .
$$

Doing some algebra we get that

$$
\psi_{z}(z)=c_{z} e^{-i(\operatorname{Im} z) \cdot x / \hbar} e^{-(x-\operatorname{Re} z)^{2} / 2 \hbar}
$$

where the constant $c_{z}$ is given by

$$
c_{z}=(2 \pi \hbar)^{-d / 2} e^{(\operatorname{Im} z)^{2} / 2 \hbar} e^{i \operatorname{Im} z \cdot \operatorname{Re} z / \hbar} .
$$

From (66) we see that $\psi_{z}$ is a Gaussian centered at the point $\operatorname{Re} z$ and multiplied by a constant and $e^{-i(\operatorname{Im} z) \cdot x / \hbar}$. A function of this sort are called Gaussian wave packet; it is the oscillating "wave" $e^{-i(\operatorname{Im} z) \cdot x / \hbar}$ multiplied by a Gaussian.

These states are very special. For example, they are "minimum uncertainty" states. This means that they give equality in the inequality of the Heisenberg uncertainty principle. One should think of $\psi_{z}$ as being the closest thing there is to a quantum state with position $\operatorname{Re} z$ and momentum $\operatorname{Im} z$. This is the idea that is intended to be conveyed by the word "coherent"-these states are as localized in phase space as is consistent with the uncertainty principle. In addition, these states behave in a very simple way with respect to the time-evolution of a quantum harmonic oscillator.

Let us express the isometricity of the Segal-Bargmann transform in terms of the coherent states. The isometricity of $C_{\hbar}$ tells us that for all $f, g \in L^{2}\left(\mathbb{R}^{d}, d x\right)$ we have

$$
\int_{\mathbb{R}^{d}} \overline{f(x)} g(x) d x=\int_{\mathbb{C}^{d}} \overline{C_{\hbar} f(z)} C_{\hbar} g(z) \nu_{\hbar}(z) d z .
$$

But by (64), $C_{\hbar} g(z)=\left\langle\psi_{z}, g\right\rangle$ and $\overline{C_{\hbar} f(z)}=\left\langle f, \psi_{z}\right\rangle$, so

$$
\int_{\mathbb{R}^{d}} \overline{f(x)} g(x) d x=\int_{\mathbb{C}^{d}}\left\langle f, \psi_{z}\right\rangle\left\langle\psi_{z}, g\right\rangle \nu_{\hbar}(z) d z .
$$

Now let $\left|\psi_{z}\right\rangle\left\langle\psi_{z}\right|$ be the operator given by

$$
\left|\psi_{z}\right\rangle\left\langle\psi_{z}\right| f=\psi_{z}\left\langle\psi_{z}, f\right\rangle,
$$


which is essentially just projection onto the state $\psi_{z}$. (The projection would have a factor of $\left\|\psi_{z}\right\|^{2}$ in the denominator.) This is part of the "Dirac notation" commonly used in physics. To understand the logic behind this notation note that $\left\langle f, \psi_{z}\right\rangle\left\langle\psi_{z}, g\right\rangle$ is just the inner product of $f$ with $\left|\psi_{z}\right\rangle\left\langle\psi_{z}\right| g$. The Dirac notion expresses the inner product with a vertical line, so $\left\langle f \mid \psi_{z}\right\rangle$ instead of $\left\langle f, \psi_{z}\right\rangle$. So in Dirac notation $\left\langle f, \psi_{z}\right\rangle\left\langle\psi_{z}, g\right\rangle$ becomes $\left\langle f \mid \psi_{z}\right\rangle\left\langle\psi_{z} \mid g\right\rangle$ which is supposed to be notationally indistinguishable from the inner product of $f$ with $\left|\psi_{z}\right\rangle\left\langle\psi_{z}\right| g$. So then (67) can be rewritten by formally bringing the integral inside the inner product to give

$$
\langle f, g\rangle=\left\langle f,\left(\int_{\mathbb{C}^{d}}\left|\psi_{z}\right\rangle\left\langle\psi_{z}\right| \nu_{\hbar}(z) d z\right) g\right\rangle .
$$

If this holds for all $f$ and $g$ then the operator inside the parentheses on the right in (68) must be the identity operator:

$$
\int_{\mathbb{C}^{d}}\left|\psi_{z}\right\rangle\left\langle\psi_{z}\right| \nu_{\hbar}(z) d z=I .
$$

Equation (69) is called a resolution of the identity. Note that both sides are operators in $L^{2}\left(\mathbb{R}^{d}, d x\right)$, even though the integral is over $\mathbb{C}^{d}$. This is because the coherent states $\psi_{z}$ are elements of $L^{2}\left(\mathbb{R}^{d}, d x\right)$, with parameter $z$ in $\mathbb{C}^{d}$. Formally, (69) is equivalent to the isometricity of the Segal-Bargmann transform. This resolution of the identity first appears in the 1960 paper of John Klauder $[$. (Klauder uses a different normalization.) The resolution of the identity is often a useful way to think about the Segal-Bargmann transform (or its generalizations). The weakness of this point of view is that there is no straightforward way to express the surjectivity of the Segal-Bargmann transform (that it maps onto the space of square-integrable holomorphic functions) in terms of the coherent states. Still, it is useful to be able to go back and forth between the transform point of view and the coherent state point of view.

One can think about Toeplitz operators in terms of the coherent states $\phi_{z} \in$ $\mathcal{H} L^{2}\left(\mathbb{C}^{d}, \nu_{\hbar}\right)$. If $f$ is a not-necessarily-holomorphic function on $\mathbb{C}^{d}$, then the Toeplitz operator $T_{f}$ on $\mathcal{H} L^{2}\left(\mathbb{C}^{d}, \nu_{\hbar}\right)$ may be expressed as

$$
T_{f}=\int_{\mathbb{C}^{d}} f(z)\left|\phi_{z}\right\rangle\left\langle\phi_{z}\right| \nu_{\hbar}(z) d z .
$$

I leave it as an (instructive) exercise to the reader to verify this expression, using properties of Toeplitz operators and of the coherent states. Note that taking $f \equiv 1$ in $(70)$ gives the analog of $\sqrt{69})$ in $\mathcal{H} L^{2}\left(\mathbb{C}^{d}, \nu_{\hbar}\right)$.

Numerous other kinds of coherent states have been considered. See for example the books [KS] and [P].

\subsection{Kähler quantization}

A Kähler manifold is a complex manifold $M$ with a symplectic structure (i.e., a nice Poisson bracket) in which the two structures satisfy a natural compatibility 
condition. The simplest example is $\mathbb{C}^{d}$ itself. The theory of geometric quantization [W] gives you a way of associating Hilbert space with certain Kähler manifolds. In the case of $\mathbb{C}^{d}$, the resulting Hilbert space is (or can be identified with) the Segal-Bargmann space. In general the Hilbert space is a space of $L^{2}$ holomorphic sections of a holomorphic line bundle over $M$. In the case of $\mathbb{C}^{d}$ this line bundle is holomorphically trivial, which means that the Hilbert space can be identified with an $L^{2}$ space of holomorphic functions-the SegalBargmann space. So these Hilbert spaces of holomorphic sections of line bundles should be thought of as generalizations of the Segal-Bargmann space, in which $\mathbb{C}^{d}$ is replaced by some other Kähler manifold. Another example is the unit disk (with an $S U(1,1)$-invariant symplectic structure) in which case the space of sections may be identified with one of the weighted Bergman spaces. Note that Planck's constant is a parameter in the geometric quantization scheme; different values of $\hbar$ give different values of $a$ in the weighted Bergman spaces. I have already mentioned this example in connection with generalized canonical commutation relations. However, the method of Kähler quantization applies to arbitrary Kähler manifolds, not assumed to have any symmetry condition. One interesting case is that of compact Kähler manifolds. In this case the quantum Hilbert space is finite-dimensional, reflecting the finite size of the classical phase space. There is much interesting topology in the line bundles in this case.

These $L^{2}$ spaces of holomorphic sections allow much of the same structure as our spaces $\mathcal{H} L^{2}(U, \alpha)$. In particular, pointwise evaluation is continuous, so there is a reproducing kernel and the holomorphic subspace is a closed subspace. So you have coherent states as above and you can define Toeplitz operators in a similar fashion to what we consider.

For a sampling of papers on this subject, see the works of Klimek and Lesniewski [KL1, KL2], Coburn [C], Bordemann, Meinrenken, and Schlichenmaier [BMS], Borthwick, Lesniewski, and Upmeier [BLU], and Borthwick, Paul, and Uribe [BPU]. The expository paper [Bd] gives a (fairly) gentle introduction to some of the techniques. As always, the book of Woodhouse [W] gives valuable background material. (But there is a very large amount of information in [W] and it is not always easy to extract what is relevant to a particular application.)

\section{References}

[BSZ] J. Baez, I. Segal, and Z. Zhou, "Introduction to Algebraic and Constructive Quantum Field Theory," Princeton Univ. Press, Princeton, NJ, 1992.

[B1] V. Bargmann, On a Hilbert space of analytic functions and an associated integral transform, Part I, Comm. Pure Appl. Math. 14 (1961), 187-214.

[B2] V. Bargmann, Remarks on a Hilbert space of analytic functions, Proc. Nat. Academy Sci. U.S.A. 48 (1962), 199-204.

[B3] V. Bargmann, Acknowledgment, Proc. Nat. Academy Sci. U.S.A. 48 (1962), 2204. 
[B4] V. Bargmann, On unitary ray representations of continuous groups, Ann. Math. 59 (1954), 1-46.

[B5] V. Bargmann, Irreducible unitary representations of the Lorentz group, Ann. Math. 48 (1947), 568-640.

[BMS] M. Bordemann, E. Meinrenken, and M. Schlichenmaier, Toeplitz quantization of Kähler manifolds and $g l(N), N \rightarrow \infty$ limits, Comm. Math. Phys. 165 (1994), 281-296.

[Bo] D. Borthwick, Microlocal techniques for semiclassical problems in geometric quantization, Contemporary Math. 214 (1998), 23-37.

[BLU] D. Borthwick, A. Lesniewski, and H. Upmeier, Non-perturbative deformation quantization of Cartan domains, J. Funct. Anal. 113 (1993), $153-176$.

[BPU] D. Borthwick, T. Paul, and A. Uribe, Legendrian distributions with applications to relative Poincaré series, Invent. Math. 122 (1995), 359-402.

[C] L. Coburn, Deformation estimates for the Berezin-Toeplitz quantization, Comm. Math. Phys. 149 (1992), 415-424.

[DH] B. Driver and B. Hall, Yang-Mills theory and the Segal-Bargmann transform, Commun. Math. Phys. 201 (1999), 249-290.

[F] G. Folland, "Harmonic analysis in phase space," Princeton Univ. Press, Princeton, N.J., 1989.

[Go] M. Gotay, On the Groenewold-Van Hove problem for $\mathbb{R}^{n}$, J. Math. Phys. 40 (1999), 2107-2116.

[GP] S. Graffi and T. Paul, The Schrödinger equation and canonical perturbation theory, Comm. Math. Phys. 108 (1987), 25-40.

[Gr] L. Gross, Abstract Wiener spaces, in "Proceedings of the Fifth Berkeley Symposium on Mathematical Statistics and Probablility," Vol. II, Univ. of California Press, 1967.

[GM] L. Gross and P. Malliavin, Hall's transform and the Segal-Bargmann map, in "Itô's stochastic calculus and probability theory," (M. Fukushima, N. Ikeda, H. Kunita, and S. Watanabe, Eds.), pp. 73-116. Springer-Verlag, Berlin/New York, 1996.

[H1] B. Hall, The Segal-Bargmann "coherent state" transform for compact Lie groups, J. Funct. Anal. 122 (1994), 103-151.

[H2] B. Hall, The inverse Segal-Bargmann transform for compact Lie groups, J. Funct. Anal., 143 (1997), 98-116. 
[H3] B. Hall, Phase space bounds for quantum mechanics on a compact Lie group, Comm. Math. Phys., 184 (1997), 233-250.

[H4] B. Hall, Quantum mechanics in phase space, Contemp. Math. 214 (1998), 47-62.

[H5] B. Hall, A new form of the Segal-Bargmann transform for Lie groups of compact type, Canad. J. Math. 51 (1999), 816-834.

[H6] B. Hall, Coherent states, Yang-Mills theory, and reduction, preprint. [htttp://xxx.lanl.gov, quant-ph/9911052

[H7] B. Hall, Geometric quantization and the generalized Segal-Bargmann transform, in preparation.

[HS] B. Hall and A. Sengupta, The Segal-Bargmann transform for path-groups, J. Funct. Anal. 152 (1998), 220-254.

[Ki] A. Kirillov, Merits and demerits of the orbit method. Bull. Amer. Math. Soc. (N.S.) 36 (1999), 433-488.

[K] J. Klauder, The action option and a Feynman quantization of spinor fields in terms of ordinary c-numbers, Ann. Phys. 11 (1960), 123.

[KS] J. Klauder and B. Skagerstam, "Coherent States," World Scientific, Cleveland, 1985.

[KL1] S. Klimek and A. Lesniewski, Quantum Riemann surfaces I. The unit disc, Comm. Math. Phys. 146 (1992), 103-122.

[KL2] S. Klimek and A. Lesniewski, Quantum Riemann surfaces: II. The discrete series, Lett. Math. Phys. 24 (1992), 125-139.

[Ku] H.-H. Kuo, "Gaussian measures in Banach spaces," Lecture Notes in Mathematics, Vol. 463. Springer-Verlag, Berlin-New York, 1975.

[PU] T. Paul and A. Uribe, A construction of quasimodes using coherent states, Ann. Inst. Henri Poincaré 59 (1993), 357-381.

[P] A. Perelomov, "Generalized coherent states and their applications." Texts and Monographs in Physics. Springer-Verlag, Berlin-New York, 1986.

[RS] M. Reed and B. Simon, "Methods of Modern Mathematical Physics, I: Functional Analysis," Academic Press, New York, London, 1972.

[S1] I. Segal, Mathematical problems of relativistic physics, Chap. VI, in "Proceedings of the Summer Seminar, Boulder, Colorado, 1960, Vol. II." (M. Kac, Ed.). Lectures in Applied Mathematics, American Math. Soc., Providence, Rhode Island, 1963.

[S2] I. Segal, Mathematical characterization of the physical vacuum for a linear Bose-Einstein field, Illinois J. Math. 6 (1962), 500-523. 
[S3] I. Segal, The complex wave representation of the free Boson field, in "Topics in functional analysis: Essays dedicated to M.G. Krein on the occasion of his 70th birthday" (I. Gohberg and M. Kac, Eds). Advances in Mathematics Supplementary Studies, Vol. 3, pp. 321-343. Academic Press, New York, 1978.

[Sh] I. Shigekawa, Itô-Wiener expansions of holomorphic functions on the complex Wiener space, in "Stochastic Analysis" (E. Mayer-Wolf, E. Merzbach, and A. Schwartz, Eds.) pp. 459-473, Academic Press, New York, 1991.

[Su] H. Sugita, Properties of holomorphic Wiener functions-skeleton, contraction, and local Taylor expanions, Prob. Theory and Related Fields, 100 (1994), 117-130.

[Th] W. Thirring, "A Course in Mathematical Physics, I: Classical Dynamical Systems," Springer-Verlag, New York, Wien, 1978.

[TW] L. Thomas and S. Wassell, Semiclassical approximation for Schrödinger operators on a two-sphere at high energy, J. Math. Phys. 36 (1995), 54805505.

[V] A. Voros, Wentzel-Kramers-Brillouin method in the Bargmann representation, Phys. Rev. A 40 (1989), 6814-6825.

[W] N. Woodhouse, "Geometric Quantization," Second Edition, Oxford Univ. Press, Oxford, New York, 1991. 